\title{
Performance Evaluation of Multimedia Broadcast Applications with Network Coding in Ad hoc Wireless Mesh Networks
}

By

\author{
Basil Saeed
}

A thesis submitted to The Faculty of Graduate Studies and Research in partial fulfillment of the degree requirements of

Master of Applied Science in Electrical and Computer Engineering

Ottawa-Carleton Institute for Electrical \& Computer Engineering

Department of Systems and Computer Engineering

Carleton University

Ottawa, Ontario, Canada

May 2011

(C) Copyright 2011 
Library and Archives

Canada

Published Heritage

Branch

395 Wellington Street

Ottawa ON K1A ON4

Canada
Bibliotheque et

Archives Canada

Direction du

Patrimoine de l'édition

395 , rue Wellington

Ottawa ON K1A ON4

Canada
Your file Votre référence

ISBN: 978-0-494-81717-9

Our file Notro référence

ISBN: 978-0-494-81717-9
NOTICE:

The author has granted a nonexclusive license allowing Library and Archives Canada to reproduce, publish, archive, preserve, conserve, communicate to the public by telecommunication or on the Internet, loan, distribute and sell theses worldwide, for commercial or noncommercial purposes, in microform, paper, electronic and/or any other formats.

The author retains copyright ownership and moral rights in this thesis. Neither the thesis nor substantial extracts from it may be printed or otherwise reproduced without the author's permission.
AVIS:

L'auteur a accordé une licence non exclusive permettant à la Bibliothèque et Archives Canada de reproduire, publier, archiver, sauvegarder, conserver, transmettre au public par télécommunication ou par l'Internet, prêter, distribuer et vendre des thèses partout dans le monde, à des fins commerciales ou autres, sur support microforme, papier, électronique et/ou autres formats.

L'auteur conserve la propriété du droit d'auteur et des droits moraux qui protège cette thèse. Ni la thèse ni des extraits substantiels de celle-ci ne doivent être imprimés ou autrement reproduits sans son autorisation.
In compliance with the Canadian Privacy Act some supporting forms may have been removed from this thesis.

While these forms may be included in the document page count, their removal does not represent any loss of content from the thesis.
Conformément à la loi canadienne sur la protection de la vie privée, quelques formulaires secondaires ont été enlevés de cette thèse.

Bien que ces formulaires aient inclus dans la pagination, il n'y aura aucun contenu manquant.

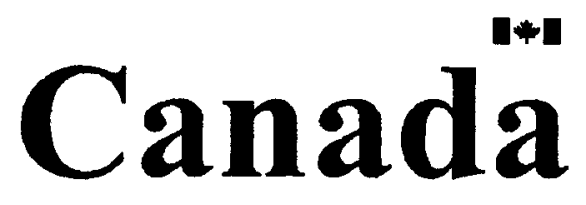


The undersigned recommend to the Faculty of Graduate Studies and Research acceptance of the thesis entitled

\title{
Performance Evaluation of Multimedia Broadcast Applications with Network Coding in Ad hoc Wireless Mesh Networks
}

\author{
submitted by Basil Saeed \\ in partial fulfillment of the requirements of the degree of \\ Master of Applied Science in Electrical and Computer Engineering
}




\begin{abstract}
Over the past decades, we have witnessed an explosive growth in the use of the multimedia broadcast applications mobile and static devices. Multimedia applications demand new approaches to audio and video transmissions to meet the growing traffic volume, quantity and quality of audio/video traffic, and users' needs. This research studies the effect of network coding on multimedia broadcast applications. Network coding has the potential to improve the performance of multimedia applications in terms of Packet Delivery Ratio (PDR), latency and jitter. This thesis examines several types of network coding protocols that work well with single and multiple sources ad hoc wireless mesh networks and compares their performance results on multimedia broadcast applications with optimized broadcast protocols with a number of simulations on NS-2. The simulation results demonstrate how Random Linear Network Coding (RLNC) can improve the overall performance results of multimedia broadcast applications in low and high density networks.
\end{abstract}




\section{ACKNOWLEDGEMENTS}

First and foremost I offer my sincerest gratitude to my supervisor, Professor Chung-Horng Lung, who has supported me throughout my thesis with his patience and knowledge whilst allowing me the room to work in my own way. I attribute the level of my Masters degree to his encouragement and effort and without him this thesis, too, would not have been completed or written. One simply could not wish for a better or friendlier supervisor.

I also would like to thank Professor Thomas Kunz for providing me with NS-2 codes for SMF and PDP. I also would like to thank my friends and colleagues who were always there to extend support and motivation. I would like to thank the SCE department and overall the Carleton University for all their support. This work was partially funded by EION Wireless, Canada and I would like to thank EION Wireless for their support.

Also, I would like to especially thank my parents whose prayers are always there for me and it's due to their prayers and efforts which have led me to success in life. I would like to thank my fiancé who was always there to support me in all circumstances and situations. Finally, I would like to thank my sister and brother who were always encouraging me during my study. 


\section{TABLE OF CONTENTS}

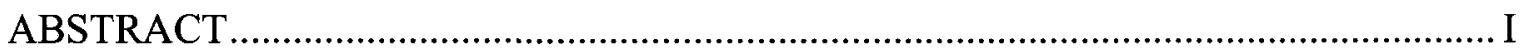

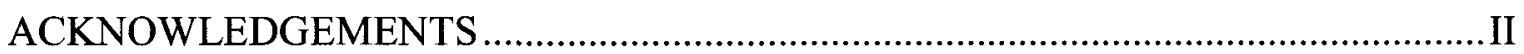

TABLE OF CONTENTS .......................................................................................

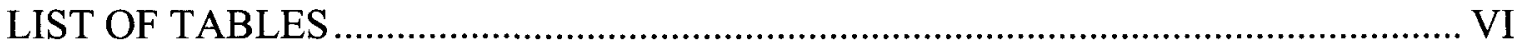

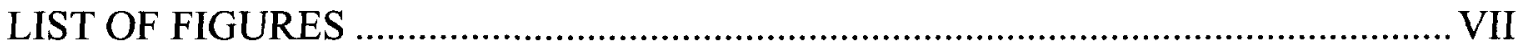

LIST OF ABBREVIATIONS ........................................................................... XI

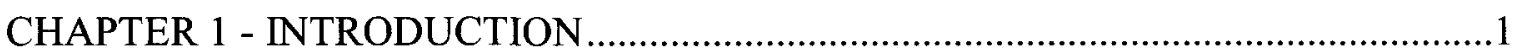

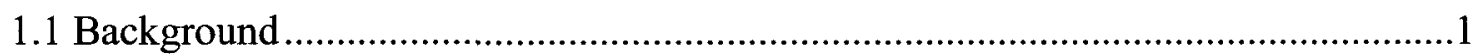

1.2 Multimedia Broadcasting ..........................................................................

1.3 Wireless Networks with Network Coding .......................................................2

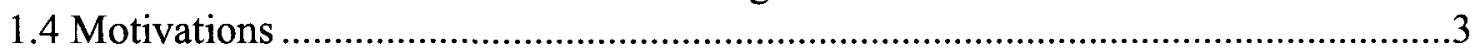

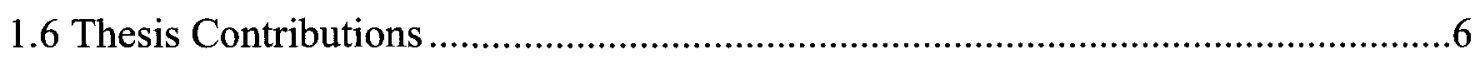

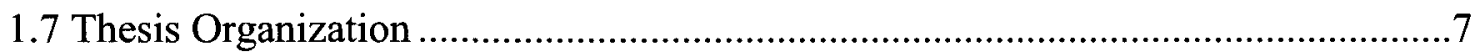

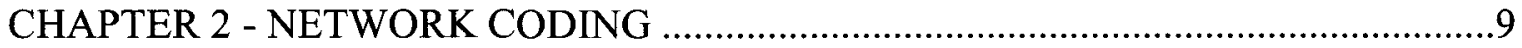

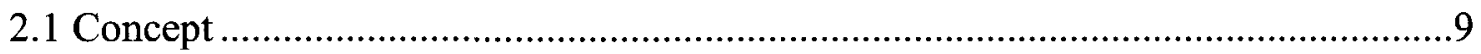

2.2 Types of Network Coding ............................................................................. 10

2.2.1 Physical Linear Network Coding (PLNC) ..............................................

2.2.1.1 Amplify and Forward (AF) ..........................................................11

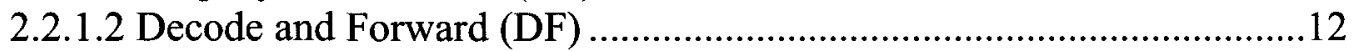

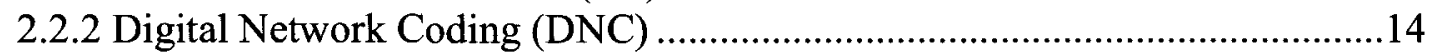

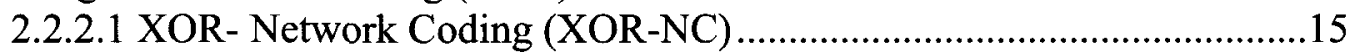

2.2.2.2 Reed-Solomon Network Coding (RS-NC) ........................................18

2.2.2.3 Random Linear Network Coding (RLNC) ......................................20

2.3 Benefits of Network Coding on Wireless Networks .........................................24

CHAPTER 3 - RELATED WORK ON MULTIMEDIA APPLICATION WITH NETWORK CODING AND NETWORK CODING BENEFITS .......................27

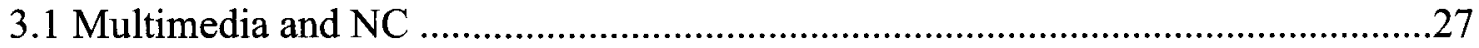

3.1.1 Peer-to-Peer Streaming With Hierarchical Network Coding.........................27

3.1.2 Network Coding for Peer-Assisted Multimedia Streaming..........................28

3.1.3 Network Coding for Wireless Video Communication ..................................28

3.1.4 An Approach of Scalable MPEG-4 Video Bitstreams with Network Coding for P2P Swarming System .........................................................29

3.1.5 Minimizing Delay for Video Conference with Network Coding ....................30

3.1.6 Priory Ordering \& Packetization for Scalable Video Multicast with Network Coding. 
3.1.7 Secure Network Coding for Multi-Resolution Wireless Video Streaming .....31

3.1.8 Experimental Measurement for VoIP with Network coding in IEEE 802.11

3.1.9 CODECAST: A NETWORK-CODING-BASED AD HOC MULTICAST PROTOCOL

3.1.10 Reliable Wireless Broadcast with Random Network Coding for Realtime Applications .35

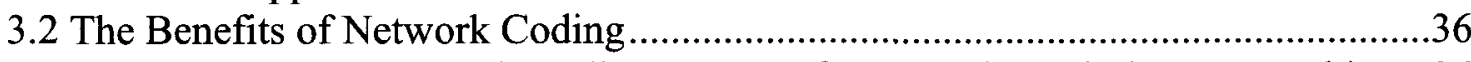

3.2.1 A Proactive Network Coding Strategy for Pervasive Wireless Networking...36

3.2.2 AN Efficient Operation of Wireless Packet Networks Using Network Coding. 37

3.2.3 On the Scaling Law of Network Coding Gains in Wireless Networks .............38

3.2.4 Network Coding Performance for Reliable Multicast. 39

3.2.5 On the Performance of Random Linear Network Coding in Relay Networks .39

3.2.6 Wireless Network Coding with Improved Opportunistic Listening. 40

3.2.7 Broadcasting in Multihop Mobile Tactical Networks: To Network Code or Not. .40

3.2.8 On MAC Scheduling and Packet Combination Strategies for Practical Random Network .41

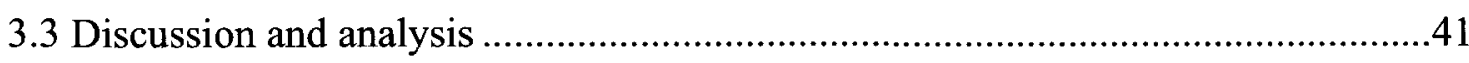

CHAPTER 4 - PROTOCOLS AND WIRELESS MODEL UNDER STUDY ...............45

4.1 Simplified Multicast Forwarding (SMF) Protocol..........................................45

4.2 Partial Dominant Pruning (PDP) Protocol ............................................................47

4.3 Random Linear Network Coding Protocol ........................................................49

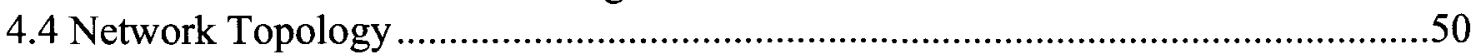

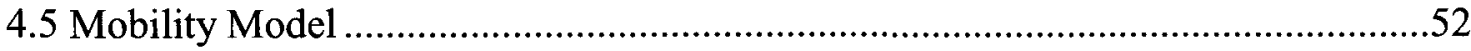

CHAPTER 5 - SIMULATION DESIGN AND IMPLEMENTATION .........................54

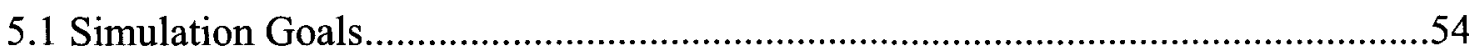

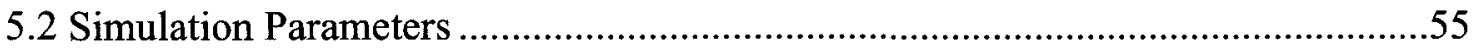

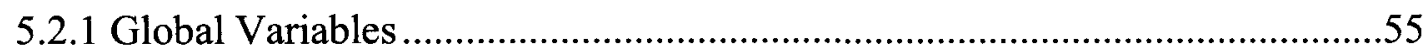

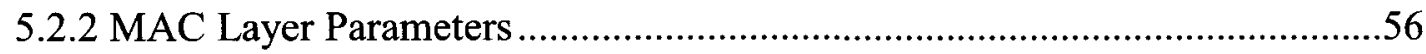

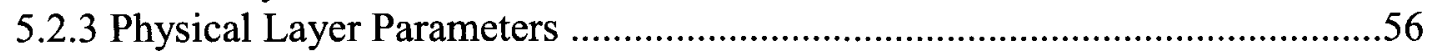

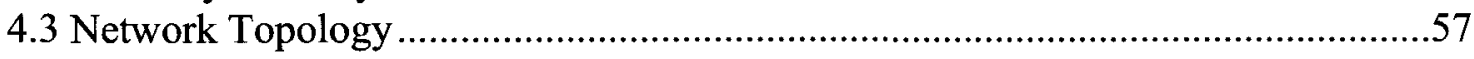

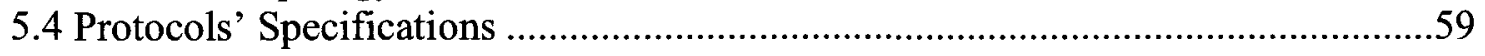

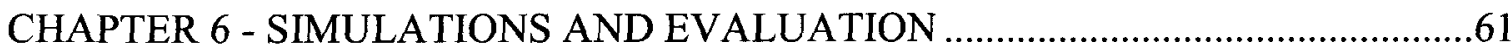

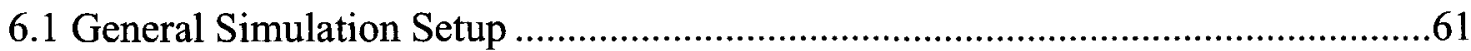

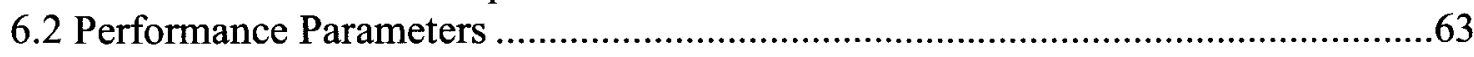

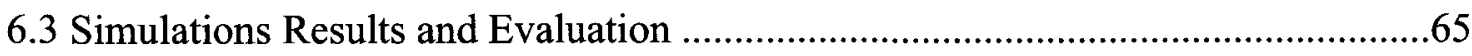

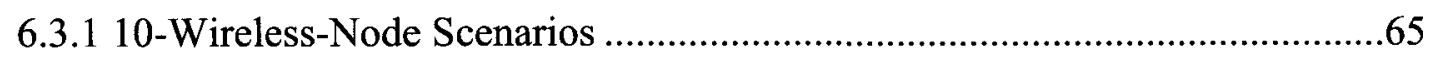

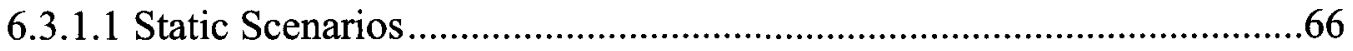

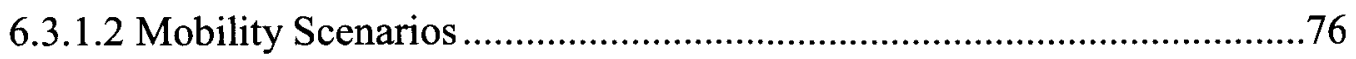

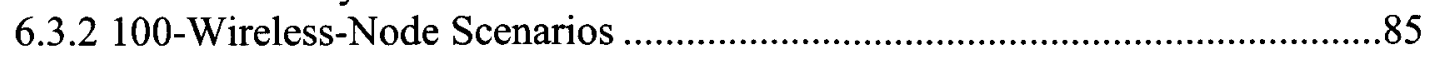

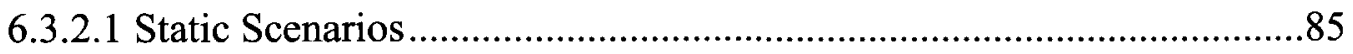




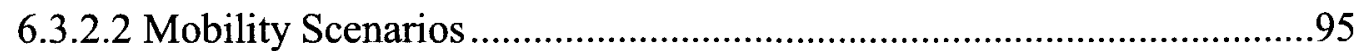

CHAPTER 7 - CONCLUSION AND FUTURE WORK …………...............................105

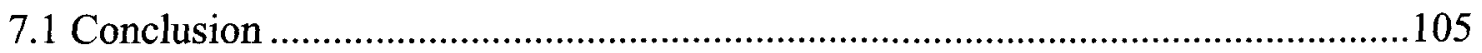

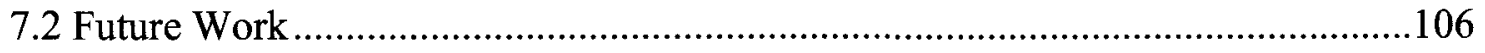

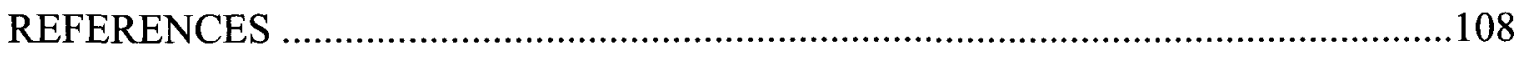

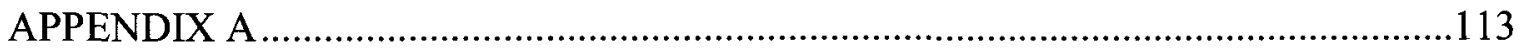




\section{LIST OF TABLES}

Table 2.1: PLNC QPSK Mapping [13] ............................................................ 13

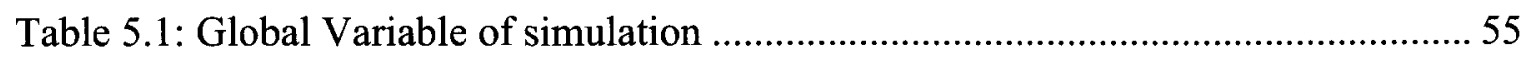

Table 5.2: MAC Layer Variable of simulation ..................................................... 56

Table 5.3: Physical Layer Variable of simulation ............................................... 56

Table 5.4: Antenna Variable of simulation......................................................... 57

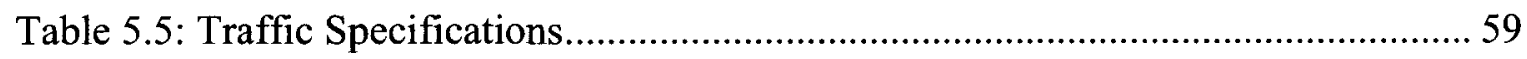

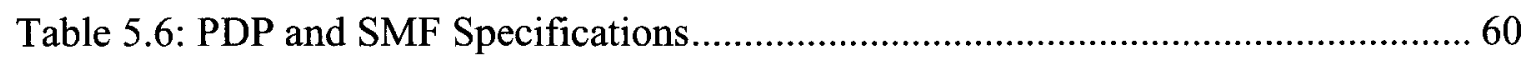

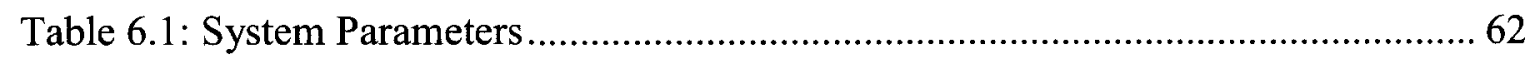




\section{LIST OF FIGURES}

Figure 1.1: Mobile video/TV users from 2004-2014 [4] ....................................... 4

Figure 1.2: Audio Streaming Growth [5] ....................................................... 4

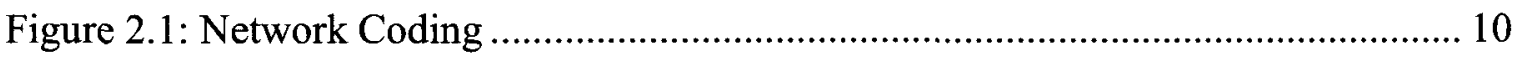

Figure 2.2: Physical-Layer Network Coding [13] ............................................. 11

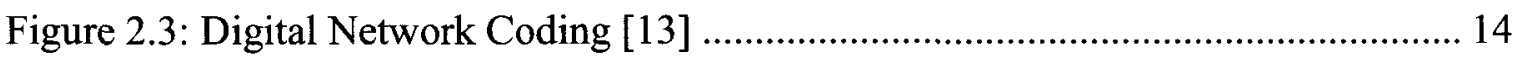

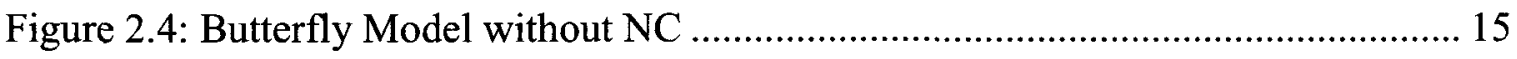

Figure 2.5: Butterfly Model with NC ............................................................... 16

Figure 2.6: Bob and Alice Network without XOR-NC ….................................... 17

Figure 2.7: Bob and Alice Network with XOR-NC ....................................... 18

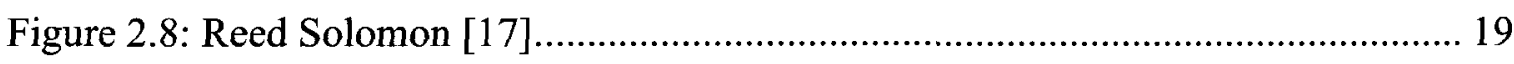

Figure 2.9: Random Linear Network Coding Diagram [22] .................................... 22

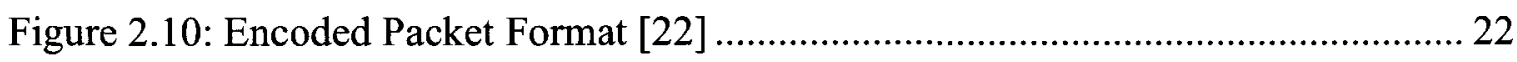

Figure 2.11: Regular Transmission vs. NC transmission .......................................... 25

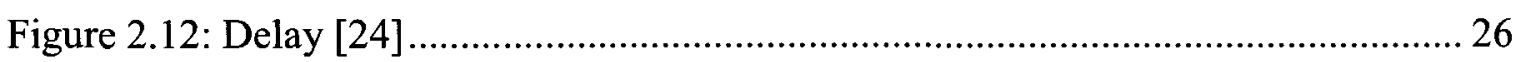

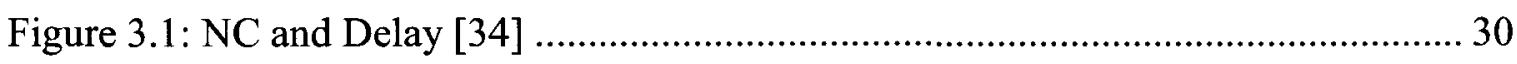

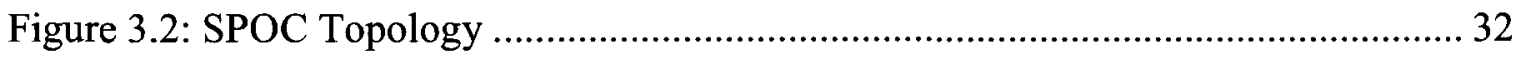

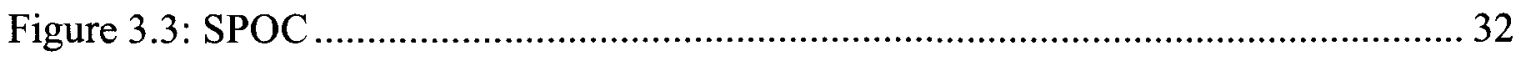

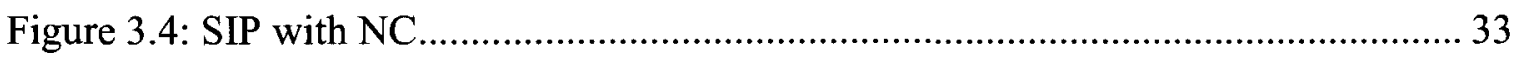

Figure 4.1: SMF Components......................................................................... 45

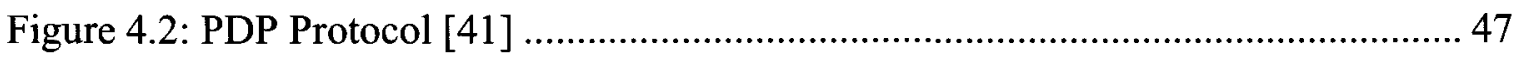

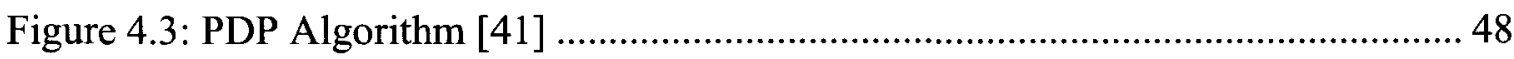

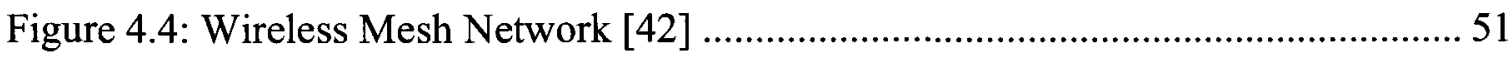

Figure 4.5: Random Waypoint Mobility Model [44]............................................. 52 


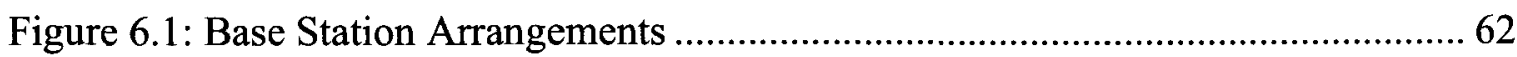

Figure 6.2: PDR for Audio Streaming (Static Scenario - Low Density)............................ 66

Figure 6.3: PDR for Video Streaming (Static Scenario - Low Density)............................ 67

Figure 6.4: PDR for SDTV (Static Scenario - Low Density)............................................. 67

Figure 6.5: PDR for HDTV (Static Scenario - Low Density) ............................................. 68

Figure 6.6: Latency for Audio Streaming (Static Scenario - Low Density) ..................... 71

Figure 6.7: Latency for Video Streaming (Static Scenario - Low Density)...................... 72

Figure 6.8: Latency for SDTV (Static Scenario - Low Density) ……………………….... 72

Figure 6.9: Latency for HDTV (Static Scenario - Low Density)....................................... 73

Figure 6.10: Jitter for Audio Streaming (Static Scenario - Low Density) ........................ 74

Figure 6.11: Jitter for Video Streaming (Static Scenario - Low Density)......................... 74

Figure 6.12: Jitter for SDTV (Static Scenario - Low Density) ………………………..... 75

Figure 6.13: Jitter for HDTV (Static Scenario - Low Density)........................................ 75

Figure 6.14: PDR for Audio Streaming (Mobile Scenario - Low Density) ....................... 77

Figure 6.15: PDR for Video Streaming (Mobile Scenario - Low Density) …………..... 78

Figure 6.16: PDR for SDTV (Mobile Scenario - Low Density) ……………………........ 79

Figure 6.17: PDR for HDTV (Mobile Scenario - Low Density) ………………….......... 79

Figure 6.18: Latency for Audio Streaming (Mobile Scenario - Low Density) ………..... 80

Figure 6.19: Latency for Video Streaming (Mobile Scenario - Low Density) ................. 81

Figure 6.20: Latency for SDTV (Mobile Scenario - Low Density) .................................... 81

Figure 6.21: Latency for HDTV (Mobile Scenario - Low Density) …………………....... 82

Figure 6.22: Jitter for Audio Streaming (Mobile Scenario - Low Density)...................... 82

Figure 6.23: Jitter for Video Streaming (Mobile Scenario - Low Density) ....................... 83

Figure 6.24: Jitter for SDTV (Mobile Scenario - Low Density) ....................................... 84

Figure 6.25: Jitter for HDTV (Mobile Scenario - Low Density) ........................................ 84 
Figure 6.26: PDR for Audio Streaming (Static Scenario - High Density) ........................ 86

Figure 6.27: PDR for Video Streaming (Static Scenario - High Density) ……………...... 87

Figure 6.27: PDR for SDTV (Static Scenario - High Density) ………………………...... 87

Figure 6.29: PDR for HDTV (Static Scenario - High Density) ………………………..... 88

Figure 6.30: Latency for Audio Streaming (Static Scenario - High Density)................... 89

Figure 6.31: Latency for Video Streaming (Static Scenario - High Density) ................... 90

Figure 6.32: Latency for SDTV (Static Scenario - High Density) ..................................... 91

Figure 6.33: Latency for HDTV (Static Scenario - High Density) ..................................... 91

Figure 6.34: Jitter for Audio Streaming (Static Scenario - High Density)........................ 93

Figure 6.35: Jitter for Video Streaming (Static Scenario - High Density)........................ 94

Figure 6.36: Jitter for SDTV (Static Scenario - High Density) ........................................... 94

Figure 6.37: Jitter for HDTV (Static Scenario - High Density) ……………………….... 95

Figure 6.38: PDR for Audio Streaming (Mobile Scenario - High Density) …................. 96

Figure 6.39: PDR for Video Streaming (Mobile Scenario - High Density)...................... 96

Figure 6.40: PDR for SDTV (Mobile Scenario - High Density) ……………………..... 97

Figure 6.41: PDR for HDTV (Mobile Scenario - High Density)....................................... 97

Figure 6.42: Latency for Audio Streaming (Mobile Scenario - High Density) ............... 99

Figure 6.43: Latency for Video Streaming (Mobile Scenario - High Density)................ 99

Figure 6.44: Latency for SDTV (Mobile Scenario - High Density) ............................... 100

Figure 6.45: Latency for HDTV (Mobile Scenario - High Density)................................ 100

Figure 6.46: Jitter for Audio Streaming (Mobile Scenario - High Density) ................... 101

Figure 6.47: Jitter for Video Streaming (Mobile Scenario - High Density) ................... 102

Figure 6.48: Jitter for SDTV (Mobile Scenario - High Density) .................................... 102

Figure 6.49: Jitter for HDTV (Mobile Scenario - High Density)..................................... 103

Figure A.1: NS Object Creation..................................................................................... 113 
Figure A.2: Trace File Creation ......................................................................... 113

Figure A.3: God and Topology Creation ........................................................................ 113

Figure A.4: Base Station and Nodes Creation .......................................................... 113

Figure A.5: PDP Base Station and Nodes Creation ...................................................... 114

Figure A.6: Audio Streaming Traffic..................................................................... 114

Figure A.7: PDP and SMF Broadcast Address and Port .............................................. 114

Figure A.8: Start and Stop Traffic Broadcasting ........................................................ 114

Figure A.9: PDP Specifications …………………….............................................. 115

Figure A.10: RLNC Specifications.......................................................................... 115

Figure A.11: RLNC Specifications Values............................................................... 115 


\section{LIST OF ABBREVIATIONS}

\begin{tabular}{|l|l|}
\hline \multicolumn{1}{|c|}{ Symbol } & \multicolumn{1}{c|}{ Abbreviation } \\
\hline AF & Amplified and Forward \\
\hline ARQ & Automatic Repeat request \\
\hline ARQ-E & Enhanced ARQ \\
\hline ARQ-SPR & Path Routing ARQ \\
\hline BS & Base Station \\
\hline CDN & Content Delivery Network \\
\hline COPE & Opportunistic Coding \\
\hline CSMA & Carrier Sense Multiple Access \\
\hline DCAR & Distributed Coding Aware Routing \\
\hline DF & Decode and Forward \\
\hline DNC & Digital Network Coding \\
\hline DP & Dominant Pruning \\
\hline DPD & Duplicate Packet Detection \\
\hline EM & ElectroMagnatic \\
\hline FEC & Forward Error Correction \\
\hline FGS & Fine Granularity Scalable \\
\hline GF & Galois Field \\
\hline God & General Operations Director \\
\hline HDTV & High Definition TV \\
\hline IP & Internet Protocol \\
\hline LTE & Long Time Evolution \\
\hline MAC & Medium Access Control \\
\hline MANET & Mobile Ad-hoc Network \\
\hline MPR & Multi Point Relay \\
\hline NC & Network Coding \\
\hline NCPB & Network Coded Piggy-Back \\
\hline NS-2 & Network Simulator 2 \\
\hline ODMRP & On Demand Multicast Routing Protocol \\
\hline P2P & Peer-to-Peer \\
\hline PBR & Piggy-Back Retransmission \\
\hline PDP & Partial Dominant Pruning \\
\hline PDR & Packet Delivery Ratio \\
\hline PLNC & Physical Layer Network Coding \\
\hline PST & Privacy, Security, and Trust \\
\hline QoS & quality-of-service \\
\hline RLNC & Random Linear Network Coding \\
\hline RS & Reed Solomon \\
\hline SDTV & Standard Definition TV \\
\hline SIP & Session Initiation Protocol \\
\hline SMF & Simplified Multicast Forwarding \\
\hline & \\
\hline
\end{tabular}




\begin{tabular}{|l|l|}
\hline SPOC & Secure Practical Network Coding \\
\hline SVC & Scalable Video Coding \\
\hline TV & Television \\
\hline UDP & User Datagram Protocol \\
\hline VoD & Video-on-Demand \\
\hline VoIP & Voice over IP \\
\hline WiMAX & $\begin{array}{l}\text { Worldwide Interoperability for Microwave } \\
\text { Access }\end{array}$ \\
\hline WMN & Wireless Mesh Network \\
\hline
\end{tabular}




\section{CHAPTER 1 - INTRODUCTION}

This chapter discusses the background of the network coding technique, multimedia broadcasting and wireless networks with network coding. It also explains the main motivation of the thesis and its contributions. Finally, it shows the organization of the thesis.

\subsection{Background}

Since the introduction of the network coding (NC) paradigm, many articles have been published claiming that $\mathrm{NC}$ has emerged as a promising paradigm that has the potential to increase the capacity of a network [1]. Such statements have convinced many researchers to apply the NC model into the use of several wireless applications. Nevertheless, many wireless applications require a flood of information to all nodes in the network to deliver the different types of messages, such as control and data messages. The use of a regular broadcast protocol consumes the channel's bandwidth due to the many duplicated packets which are caused by the re-broadcasted lost packets and received by all destinations. The current thesis studies several NC techniques. The thesis also attempts to find out the impact of those $\mathrm{NC}$ techniques on increasingly popular wireless multimedia applications.

\subsection{Multimedia Broadcasting}

Broadcasting is a linear transmission mechanism including audio and video traffic in real time. Several types of devices, such as televisions (TVs), radios, computers and mobile devices (smart phones) are used as receivers to gain access to a single broadcasted traffic at a time per channel with pre-scheduled starting and ending times. The users have 
control over the receivers in which they can be switched on or off as well as they have frequency tuning control. Practically, all of the contents offered by mobile, radio and TV stations today are available only in this approach. The digital multimedia broadcasting standards can support high definition television (HDTV), multiple standard definition television (SDTV) and program streams. It also supports private data applications such as broadcast duplicate transmissions, multimedia pager data burst, HTML pages, audio streaming, video streaming etc. [2].

\subsection{Wireless Networks with Network Coding}

With the explosion of Internet traffic seen over the past two decades, coupled with the ever increasing need to access critical data at any location, wireless networks have emerged to effectively communicate in an on-demand fashion from nearly any location. This new communications paradigm has a broadcast nature and presents challenges not seen in the traditional wired networks due to the nature of the wireless medium in which users must share access to: i) frequencies or ii) time-slots controlled by the Medium Access Control (MAC) model used. The challenges include low throughput, bandwidth limitations, mobility impacts, energy consumption, unreliable transmission, security issues and dead spots. Exploiting the characteristics of the wireless medium, especially the broadcast communication channel, $\mathrm{NC}$ has emerged as a promising paradigm in order to increase the capacity or the throughput of the network. NC originally was proposed by Ahlswede et al [1] for wired networks, but Li, et al [3] have presented a new focus where network coding is slowly being integrated into the wireless domain. All nodes (sending or intermediate nodes) act as a relay and also can combine several received packets into one 
or several encoded outgoing packets causing an increase in the performance of the network in terms of throughput, delay and etc.

Chapter 3 will discuss the various analytical models, simulations, and the types of protocols based on several types of NC techniques. Chapter 3 will also describe how NC can improve the throughput, security, delay and the efficiency of the network. Moreover, it will show some proposed NC protocols which have been applied to multicast and broadcast applications in some wireless networks, such as wireless multimedia applications.

\subsection{Motivations}

Over the past decades, we have witnessed an explosive growth in the use of the multimedia applications with mobile and static devices. The multimedia broadcasting in wireless networks intends to transmit concurrently identical multimedia traffic to multiple receivers. The main important trends for multimedia broadcasting are:

1. Mobile traffic is growing significantly and will be dominated by video and voice.

2. Mobile devices are getting more powerful.

3. Mobile graphics are getting better.

As a result, mobile audio, video and TV users are expected to grow rapidly as shown in Figures 1.1 and 1.2. Figure 1.1 shows the growth trend for TV and video users as it is expected to blossom to more than four hundred and fifty million by 2014 [4]. Figure 1.2 shows that sixty-nine million people in the world listening to audio streaming applications nowadays, and the growth trend for this application in the future as presented in [5]. 


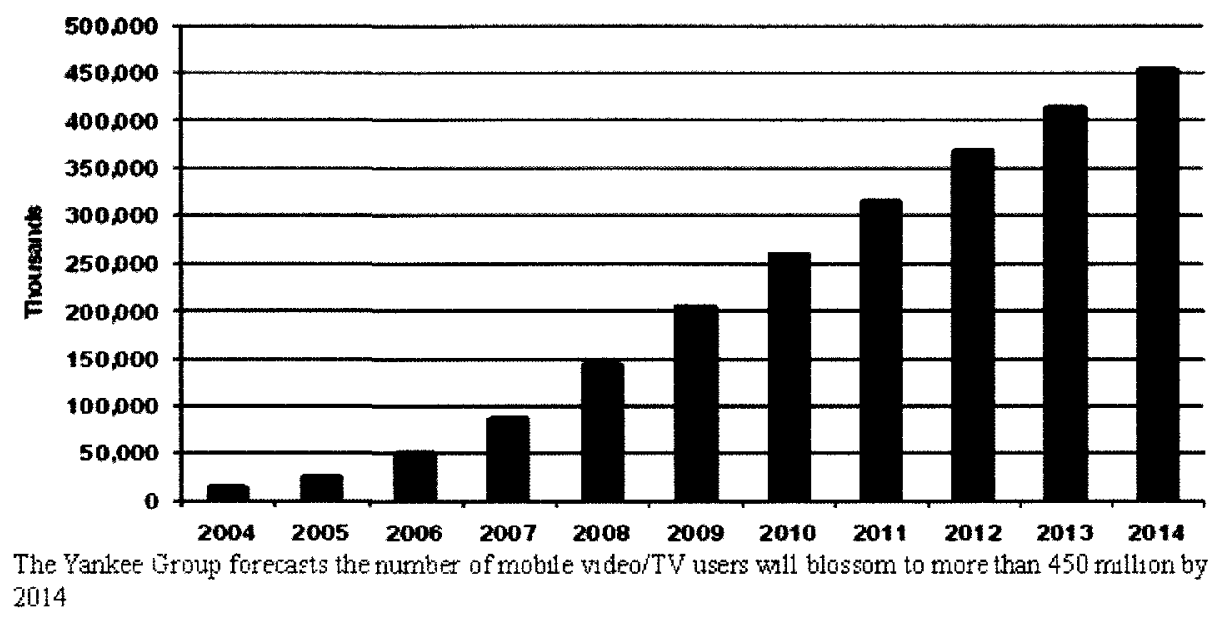

Figure 1.1: Mobile video/TV users from 2004-2014 [4]

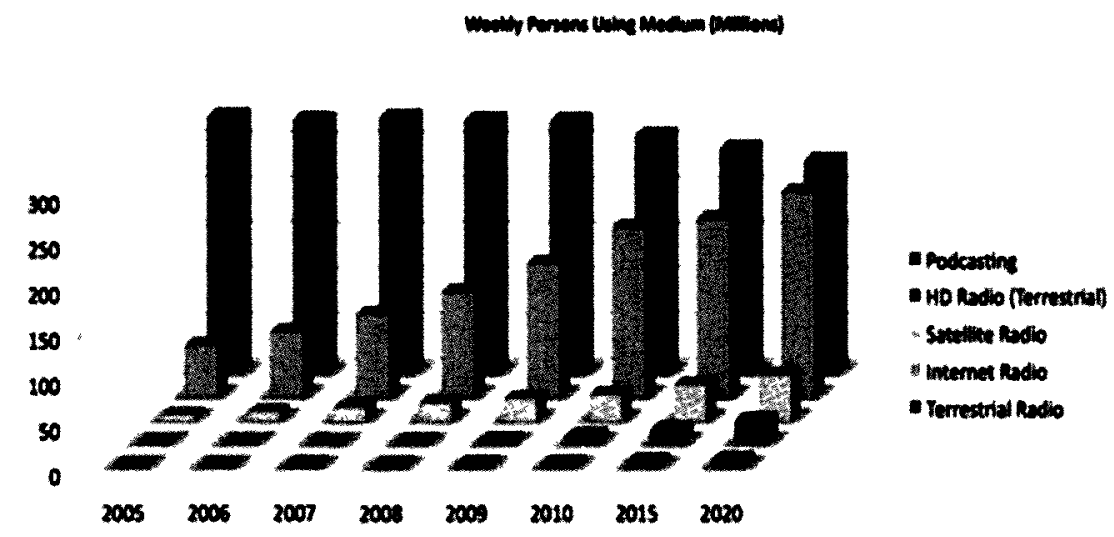

Figure 1.2: Audio Streaming Growth [5]

Due to the above mentioned growth trends of the number of mobile users, the wireless multimedia broadcasting services are faced with a number of challenges: the wireless capacities are still limited and cannot support rich multimedia traffic to mobile devices because of the increase of the video and voice qualities. This leads to increase the packet size of multimedia traffic applications (video and voice).

Furthermore, multimedia streaming traffic is extremely time-sensitive as it requires an on-time delivery of each multimedia packet before going beyond its appearance deadline. As a result, this does not provide many opportunities for delaying 
significant transmissions. The original play-out delay at the receiver essentially influences the application acceptance [6].

Due to the benefits of $\mathrm{NC}$ and the increasing popularity of multimedia applications, we were motivated to apply the $\mathrm{NC}$ mechanism to the multimedia wireless broadcasting traffic in the single source or multiple sources networks. Likewise, many proposed broadcast protocols (with or without the $\mathrm{NC}$ mechanism) have factors that help bypass the mentioned challenges. It is both theoretically and practically important to find a solution where several types of multimedia traffic can be delivered successfully to the destinations in a way that satisfies the customers.

As stated earlier, $\mathrm{NC}$ is emerging and has potential to improve performance. Therefore, in order to address the challenges of increasing demands both in terms of quantity and quality, this thesis investigates the impact of $\mathrm{NC}$ on quality-of-service (QoS) attributes (such as Packet Delivery Ratio (PDR), latency and jitter) of multimedia applications. Moreover, the thesis focuses on examining several NC protocols that work well with a single source and multiple sources ad hoc wireless mesh networks. It also compares their performances on multimedia applications with optimized broadcast protocols, e.g., Simplified Multicast Forwarding and Partial Dominant Pruning (PDP) [7].

The thesis studies seven broadcast protocols for wireless networks, which are;

1. SMF Protocol without NC

2. SMF with XOR-NC Protocol

3. SMF with Reed Solomon-NC (RS-NC) Protocol

4. PDP Protocol with NC

5. PDP with XOR-NC Protocol 
6. PDP with RS-NC Protocol

7. Random Linear Network Coding (RLNC) Protocol

All these protocols will be discussed and explained in Chapter 4. These protocols are designed to work with single source and multiple sources networks [7][8][9]. SMF Protocol without NC (option 1) and PDP Protocol without NC (option 4) are regular broadcast protocols which flood information all over the network. If the packet is received by the intermediate nodes, they will act like relays and broadcast all received packets until destinations receive their desired packets. However, RLNC and the modified versions of SMF (options 2 and 3) and PDP (options 5 and 6) protocols broadcast packets to all nodes in the networks. If the packet is received by intermediate nodes, they combine/encode received packets from several sources and broadcast the new encoded packet to all nodes in the networks. The producer of combining packets improves the PDR and reduces the delay. In other words, NC mechanism improves the overall performance of the network.

\subsection{Thesis Contributions}

Various theoretical and analytical models have been proposed using NC techniques and their performances have been evaluated [10][11][12]. Not many studies have been reported in the literature on the effect of NC on multimedia broadcast communications. Thus, the main contributions of this thesis are listed as follows:

- The thesis has investigated the potential benefits of several NC techniques, such as RLNC, RS and XOR by studying the seven broadcast protocols for ad hoc wireless mesh networks which are described in Section 1.5. 
- The thesis has compared NC techniques which are described in Section 1.5 in terms of PDR, latency and jitter.

- The thesis has investigated the potential and the suitable NC technique to be used for multimedia broadcast communications between the NC techniques which are described in Section 1.5 in ad hoc wireless mesh networks.

Parts of this thesis have appeared in the following publications and submitted papers:

- B. Saeed, C.-H. Lung, and A. Srinivasan, "Audio Streaming with Network Coding", submitted to IEEE GLOBECOM, December 2011.

- B. Saeed, P. Rengaraju, C.-H. Lung, T. Kunz and A. Srinivasan, "QoS and Protection of Wireless Relay Nodes Failure Using Network Coding", accepted to International Symposium on Network Coding (NetCod), July 2011.

- M. Hay, B. Saeed, C.-H. Lung, and A. Srinivasan, "Co-Located Physical-Layer Network Coding to Mitigate Passive Eavesdropping", Proc. of the $8^{\text {th }}$ International Conference on Privacy, Security, and Trust (PST), Auguest 2010. This paper discusses NC security issues. The paper domenstrates that $\mathrm{NC}$ can decrese the evasedropping region by an extrnal entity via the use of co-located nodes.

\subsection{Thesis Organization}

The remainder of this thesis is organized as follows: Chapter 2 discusses different types of NC techniques. Also, this chapter contains a discussion on some of the benefits of $\mathrm{NC}$ mentioned in the literature. Chapter 3 discusses some of the NC models from the literature that deals with some of the multimedia applications to wireless ad hoc networks. The main focus is on the theoretical and analytical models proposed so far. 
This chapter also briefly reviews some models that show the benefits of NC in terms of performance. Chapter 4 explains the tested protocols that are used in this thesis: RLNC, SMF with/without NC and PDP with/without NC. Chapter 5 describes the design and implementation of simulations with SMF/PDP with/without network coding and RLNC. This chapter also contains sample code from simulations and explains how it works. Chapter 6 explains the general simulation setup and various scenarios adopted for evaluation. It also shows performance results of the tested protocols in terms of PDR, delay and jitter. Chapter 7 concludes the thesis and presents some directions for future work. 


\section{CHAPTER 2 - NETWORK CODING}

This chapter discusses the concept of network coding. It also explains different types of network coding; Physical Layer Network Coding and Digital Network Coding. It also discusses the main benefits of network coding on wireless networks, such as increasing the overall throughput, decreasing the energy consumption of wireless devices, guaranteeing reliable transmissions, minimizing the delay, and etc.

\subsection{Concept}

$\mathrm{NC}$ has emerged recently as a new transmission methodology primarily designed to increase performance (i.e. network throughput) by reducing the number of transmissions required to send frames from a source to a destination. This transmission technique seeks to combine packets from different sources into one encoded packet and transmit the new encoded packet [1]. The receiver decodes the received encoded packet to recover the original packets when the receiver receives enough information that helps in the decoding procedure. As shown in Figure 2.1, node " $\mathrm{C}$ " in the three networks can perform some computation and outputs a packet which is a function of all input packets. In other words, $\mathrm{NC}$ allows information to be mixed at any node in the networks.

There has been substantial evidence to support the fact that network coding can increase network capacity in terms of PDR, latency and jitter. 


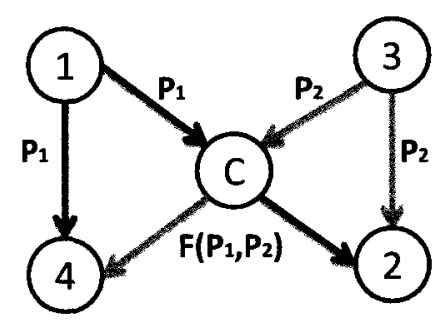

(a)

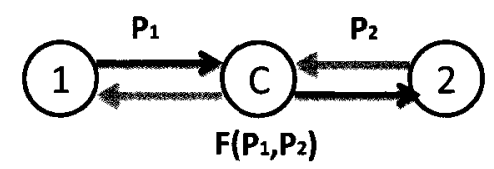

(b)

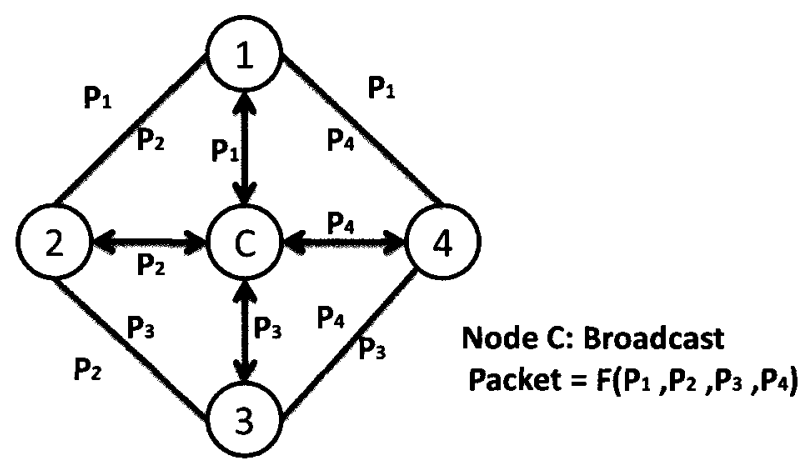

(c)

Figure 2.1: Network Coding

\subsection{Types of Network Coding}

There are two types of NC that have been found. NC can be classified into the following types;

1) Physical Linear Network Coding (PLNC)

2) Digital Network Coding (DNC)

Each of the above types is explained in details in the following sections.

\subsubsection{Physical Linear Network Coding (PLNC)}

PLNC is one type of NC that exploits the nature of the wireless medium in which electromagnetic waves from two simultaneous transmissions collide in the air, PLNC (also referred to as Analog Network Coding) seeks to allow two stations to transmit information that is received simultaneously at the relay [13]. The relay will then transmit the combined information in a broadcast manner to nodes 1 and 3 as shown in Figure 2.2. 
It can be seen that the amount of time to transmit information is reduced, thus increasing the capacity of the network. Further explanation in regards to the methods used in PLNC will be given later in sections 2.2.1.1 and 2.2.1.2.

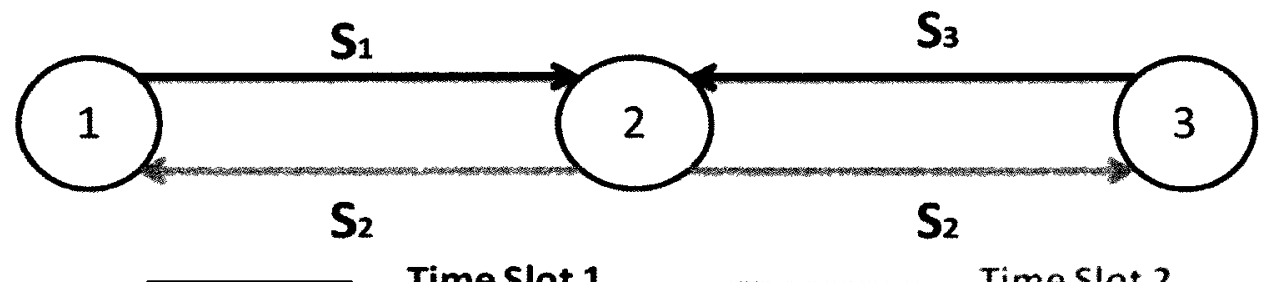

Figure 2.2: Physical-Layer Network Coding [13]

\subsubsection{Amplify and Forward (AF)}

In the AF scheme [15] and [16], the relay will not attempt to decode or interpret the two simultaneous ElectroMagnetic (EM) waves; instead after successfully receiving this combination, it will amplify the received signal and broadcast this information to nodes " 1 " and " 3 " as seen in Figure 2.2. As a result, the relay node " 2 " will receive:

$$
y_{2}[m]=h_{1} x_{1}[m]+h_{2} x_{3}+n_{2}[m]
$$

where $x_{1}$ and $x_{3}$ denote the symbols transmitted by nodes " 1 " and " 3 " respectively, $h_{1}$ and $h_{2}$ represent the complex channel coefficients for the transmit paths between nodes 1 and 3 and the relay station, and $n_{2}$ represents the noise at the relay.

The relay will then amplify this signal by a factor $\beta$ such that node " 1 " will receive:

$$
\begin{gathered}
y_{1}[m]=\beta h_{1} y_{2}[m]+n_{1}[m] \\
y_{1}[m]=\beta h_{1}^{2} x_{1}[m]+\beta h_{1} h_{2} x_{3}+\beta h_{1} n_{2}[m]+n_{1}[m]
\end{gathered}
$$

Since node " 1 " knows $x_{1}[m], h_{1}, h_{2}$ and $\beta$, it can subtract $\beta h_{1}^{2} x_{1}[m]$ from $y_{1}[m]$ giving: 


$$
y_{1}[m]=\beta h_{1} h_{2} x_{3}+\beta h_{1} n_{2}[m]+n_{1}[m]
$$

From which it can determine $x_{3}[15]$.

\subsubsection{Decode and Forward (DF)}

In the DF scheme [13], the relay performs a complete decoding of the received signal and transmits the re-encoded message. If no decoding error occurred, the relay serves as a second source to transmit the same message. In order to satisfy this, a particular modulation/demodulation mapping scheme is needed, which is based on the physical-layer modulation method used to transmit the signal (i.e. BPSK, QPSK, MSK). An example using QPSK is illustrated below. The combined band pass signal received at the relay node is:

$$
\begin{gathered}
y_{2}[m]=s_{1}[m]+s_{3}[m]=\left[a_{1} \cos (\omega t)+b_{1} \sin (\omega t)\right]+\left[a_{3} \cos (\omega t)+b_{3} \sin (\omega t)\right] \\
y_{2}[m]=\left(a_{1}+a_{3}\right) \cos (\omega t)+\left(b_{1}+b_{3}\right) \sin (\omega t)
\end{gathered}
$$

$y_{2}[m]$ is the band pass signal received by the relay node during one symbol period. Here the relay cannot decode the signals received by node " 1 " or node " 3 ". However, as long as it is able to transmit the necessary information to node " 1 " and " 3 " for the extraction of $a_{1}, a_{3}, b_{1}, b_{3}$ the end-to-end delivery of information will be successful. The relay node will then transmit a modulated mapping of the combined received signals illustrated in the Table " 1 ". Upon receiving the combined signal the relay will then transmit:

$$
x_{2}[m]=a_{2} \cos (\omega t)+b_{2} \sin (\omega t)
$$

Where $a_{2}$ and $b_{2}$ are derived from the modulation mapping based on QPSK. For example, if node " 1 " sends $s_{1}=0$ and node " 3 " sends $s_{3}=1$, then when the symbols are 
modulated at the transmitters of each node, $a_{1}=-1$ and $a_{3}=1$. This means that the relay will receive $s_{2}=0$ due to previous equation of $y_{2}[m]$ :

$$
y_{2}[m]=\left(a_{1}+a_{3}\right) \cos (\omega t)+\left(b_{1}+b_{3}\right) \sin (\omega t)=(-1+1) \cos (\omega t)+\left(b_{1}+b_{3}\right) \sin (\omega t)
$$

and transmit $a_{2}=1$ as shown in Table "l". Therefore, $x_{2}$ becomes:

$$
x_{2}[m]=a_{2} \cos (\omega t)+b_{2} \sin (\omega t)=(1) \cos (\omega t)+b_{2} \sin (\omega t)
$$

A similar mapping is performed for $b_{2}$, Nodes " 1 " and " 3 " can then derive the exchanged information bits in which node "1" will compare $a_{2}$ with $a_{1}$ to obtain $a_{3}$, or more precisely:

$$
a_{3}[m]=a_{1}[m] \oplus a_{2}[m]
$$

Where $\oplus$ denotes exclusive OR operation.

Table 2.1: PLNC QPSK Mapping [13]

\begin{tabular}{|c|c|c|c|c|c|c|}
\hline \multicolumn{3}{|c|}{ Modulation mapping at $N_{1}$ and $N_{3}$} & \multicolumn{2}{c|}{$\begin{array}{c}\text { Demodulation } \\
\text { mapping at } N_{2}\end{array}$} \\
\cline { 5 - 7 } & \multicolumn{2}{|c|}{ Input } & Output \\
\hline \multicolumn{2}{|c|}{ Input } & \multicolumn{2}{|c|}{ Output } & & $\begin{array}{c}\text { Modulation mapping } \\
\text { at } N_{2}\end{array}$ \\
\cline { 5 - 7 } & & & & Input & Output \\
\hline$s_{1}^{(n)}$ & $s_{3}^{(h)}$ & $a_{1}$ & $a_{3}$ & $a_{1}+a_{3}$ & $s_{2}^{(I)}$ & $a_{2}$ \\
\hline 1 & 1 & 1 & 1 & 2 & 0 & 1 \\
\hline 0 & 1 & 1 & 1 & 0 & 1 & 1 \\
\hline 1 & 0 & 1 & 1 & 0 & 1 & 1 \\
\hline 0 & 0 & 1 & 1 & -2 & 0 & 1 \\
\hline
\end{tabular}

Both the AF and DF PLNC techniques rely on the existence of a priori information at the receiver (i.e. the receiver knows what it has sent previously) in order for the decoding procedure to be successful. 


\subsubsection{Digital Network Coding (DNC)}

Originally the Network Coding theory was developed in the context of wire line networks where multicast packets travelling through a network were combined using techniques, such as: i) XOR, ii) Reed-Solomon (RS), or iii) Random Linear Network Coding. Further explanation in regards to the techniques used in DNC will be given later in sections 2.2.2.1, 2.2.2.2 and 2.2.2.3. Because these techniques were implemented in software, these approaches are commonly referred to as DNC.

In digital network coding, senders transmit sequentially, and routers mix the content of the packets and broadcast the mixed version. As shown in Figure 2.3, Using this approach if nodes " 1 " and " 3 " wish to exchange information it could be performed via the following: i) node "l" transmits $s_{1}$ to node " 2 ", ii) node "3" transmits $s_{3}$ to node "2", iii) node "2" performs a coding operation on the information and transmits $s_{2}$ to both nodes " 1 " and " 3 ", taking a total of 3 time slots. The received information is then decoded at the edges, an operation that is based on the type of network coding employed in the original coding operation [13].

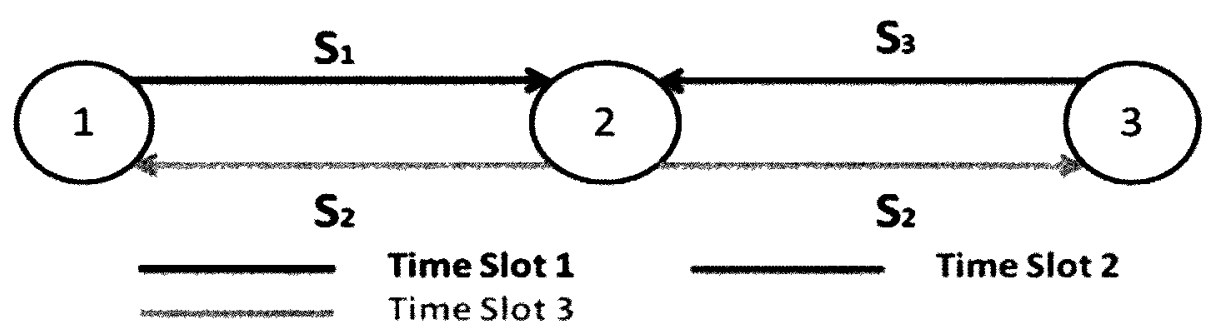

Figure 2.3: Digital Network Coding [13] 


\subsubsection{XOR- Network Coding (XOR-NC)}

XOR-NC technique is one of the simplest digital network coding techniques to encode and decode data packets. The XOR-NC technique is well explained for wired networks in the literature using several network models [1]. The most famous model to well explain XOR-NC technique is the butterfly model as shown in Figure 2.4 and Figure 2.5 .

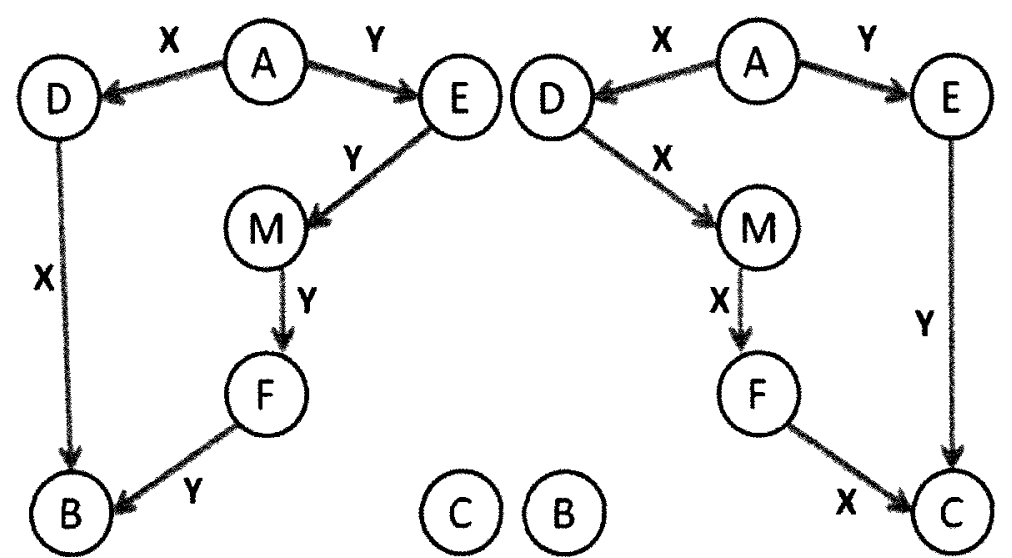

(a) (b)

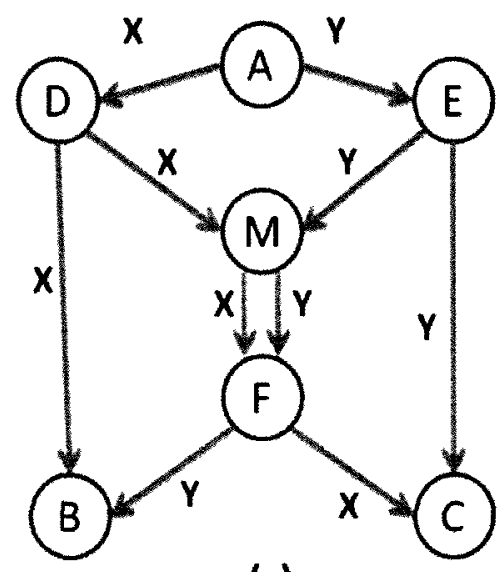

(c)

Figure 2.4: Butterfly Model without NC

Figure 2.4 shows an example of the Butterfly model without using XOR-NC. Assume that the network model at each time slot, each link can deliver one bit (Data Packet). Also, the source $A$ would like to send two different data packets to two destinations $B$ and $C$. Source $A$ can produce one bit per time slot; $A$ generates data packet $X$ and $Y$ and multicast both data packets to $D$ and $E$, respectively. As $B$ is a receiver, it uses all network resources to receive both data packets; $X$ and $Y$. As shown 2.4(a), node $B$ receives data packet $X$ through path $D B$, and receives data packet $Y$ through paths $E M$, $M F$ and $F B$. Similarly, node $C$ uses all network resources to receive both data packets; $X$ 
and $Y$. As shown 2.4(b), node $C$ receives data packet $Y$ through path $E C$, and receives data packet $X$ through paths $D M, M F$ and $F C$.

Let us assume that both receivers ( $B$ and $C$ ) want to receive data packets $X$ and $Y$ simultaneously. In other words, source $A$ wants to multi-task data packets ( $X$ and $Y$ ). Traditionally, node $M$ (shown in Figure 2.4(c)) has to make a decision either to forward data packet $X$ or $Y$. For example, if node $M$ decides to forward data packet $Y$, then receiver $B$ will receive both data packets simultaneously, but receiver $C$ will only receive data packet $Y$.

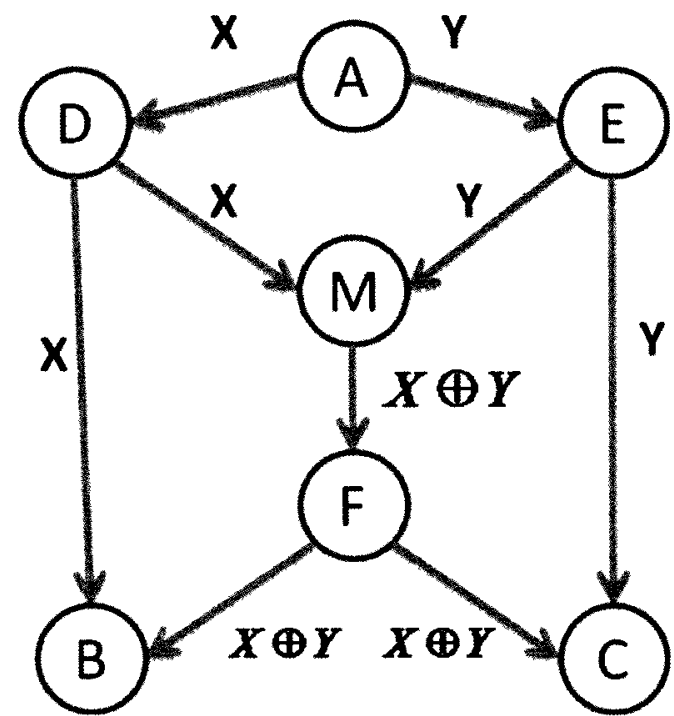

Figure 2.5: Butterfly Model with NC

The previous issue can be resolved applying the XOR-NC technique, which allows intermediate node (node $M$ in Figure 2.5) to apply XOR operation to encode both packets into one new packet; $X \oplus Y$. As shown in Figure 2.5, node $M$ forwards the XORed data packet to node $F$. Node $B$ will then receive the data packet $X$ and XORed packet $X \oplus Y$ and node $C$ will receive the data packet $Y$ and XORed packet $X \oplus Y$ through node $F$. 
Both receivers can easily decode/solve the received XORed packet, for example, node $B$ can decode $X \oplus Y$ using data packet $X$ to recover $Y ; Y=X \oplus(X \oplus Y)$. Similarly, node $C$ can decode $X \oplus Y$ using data packet $Y$ to recover $X ; X=Y \oplus(X \oplus Y)$. This example clearly shows the impact of XOR-NC by decreasing the number of time slots to deliver a packet from the source to destination. In other words, XOR-NC improves network throughput.

Nowadays, wireless networks are part of everyday lives. Thus, it is very important to figure out some solutions for the limitations of the capacity of the bandwidth. Using NC can efficiently utilize the bandwidth by increasing the throughput of dense wireless mesh networks and MANETs.

Another example of XOR-NC, this time in a wireless broadcast network, is provided in the work of Katti et al. [17] and shown in Figures 2.6 and 2.7 using Bob and Alice network model. In this figure, Figure 2.6 refers to regular approach of transmitting packets wirelessly. Bob sends his packet to the relay using one time slot. The relay then forwards the received packet to Alice using another time slot, as a result, Alice sends her packet to the relay in one time slot. Subsequently, the relay forwards the received packet to Bob using a different time slot. The total number of transmissions using the regular approach equals to 4 transmissions or time slots.

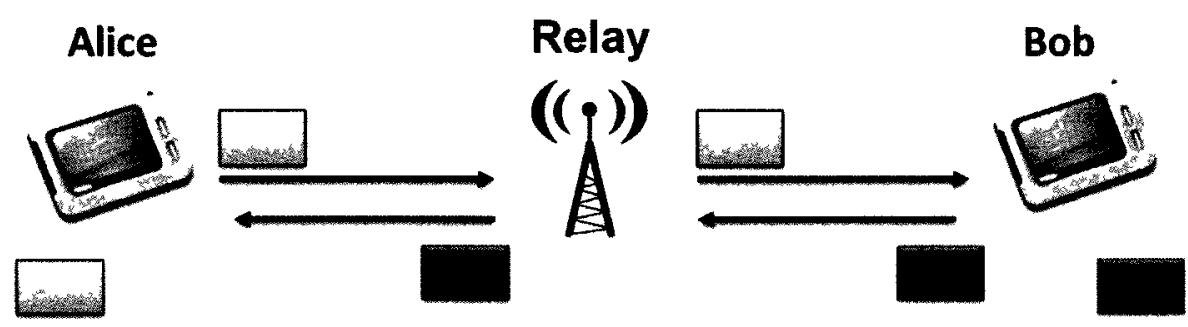

Figure 2.6: Bob and Alice Network without XOR-NC 
Figure 2.7 shows the XOR-NC approach which would be performed as following in the wireless networks. Both Bob and Alice send their packets respectively to the relay using different time slots. The relay combines the received packets into one packet using an XOR operation, and broadcasts the new packet to Bob and Alice in the third time slot. Bob and Alice can decode the information sent to them via XORing the coded packet sent by the relay with their priori packets [17]. The total number of transmissions using XOR-NC approach equals to 3 transmissions or time slots. This example shows that using the XOR-NC approach would decrease the waste of bandwidth by sending more packets using less time slots.

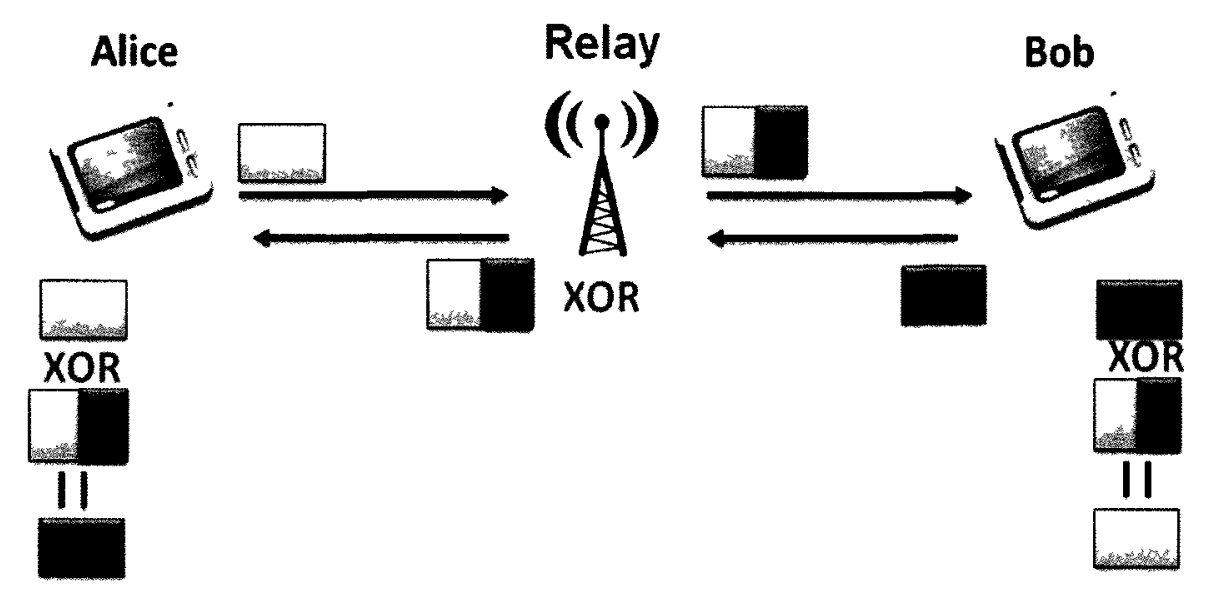

Figure 2.7: Bob and Alice Network with XOR-NC

Contemporary research has begun to formulate protocols based on XOR-NC, such as the Opportunistic Coding (COPE) [17] and the Distributed Coding Aware Routing in wireless networks (DCAR) [18].

\subsubsection{Reed-Solomon Network Coding (RS-NC)}

RS codes are mainly used as error-correcting techniques. RS codes have been used in data storage; compact discs and in the Encoding/Decoding systems that allow 
scratched CDs to sound perfect. Also, they are used in data transmission which allows high quality pictures to be sent back from Voyager II and transmits data over tens of millions of miles.

Figure 2.8 shows the processes of RS error correcting. An RS code can be represented as $R S(n, k)$ with $s$-bit symbols, where $k$ is the size of the message in symbols of $\mathrm{s}$ bits that a source wants to encode into codeword and $n$ is the size of codeword. In Figure 2.8 , the source wants to send the 1011 message to a receiver. The source encodes the 1011 message into codeword 1011010 and sends the codeword through a channel to the receiver, but during the transmission the codeword is changed from 1011010 to 1010010 which an error is occurred. At the receiver side, the decoder corrects the errors and reclaims the source message 1011 using RS code.

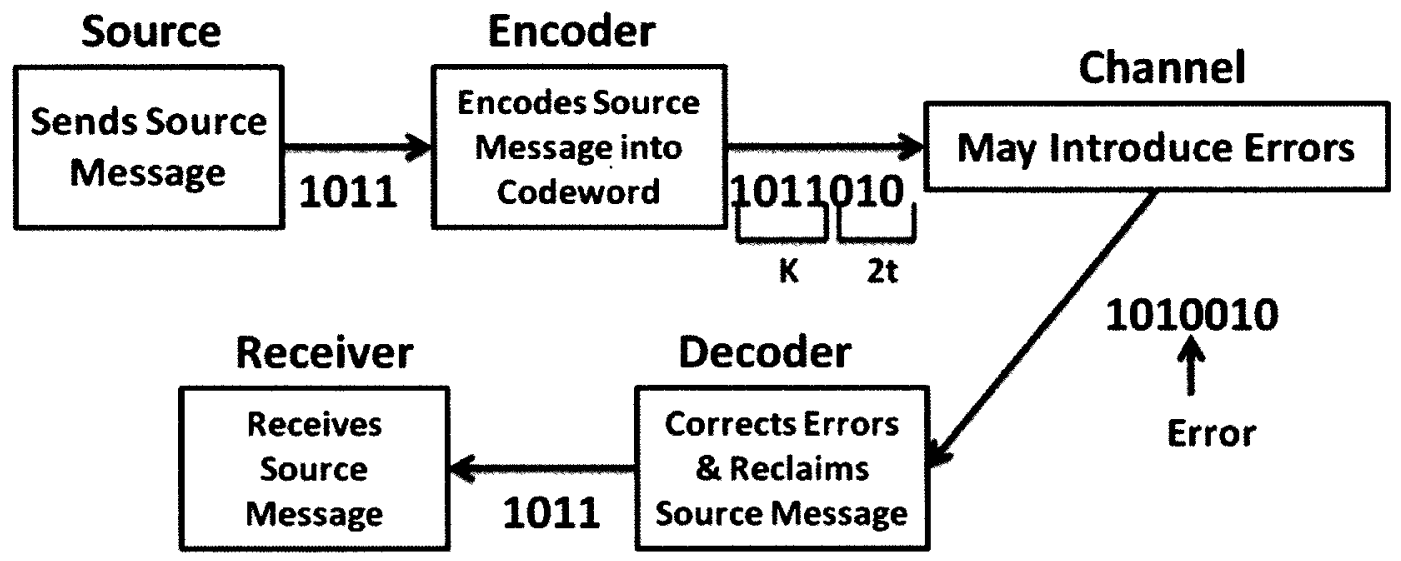

Figure 2.8: Reed Solomon [17]

Moreover, the decoder can correct up to $[(n-k+1) / 2]$ of unknown located errors. However, error location sometimes is known in advance and the errors in this case are called erasures. In the case of erasures, errors are corrected twice as fast when compared to unknown location errors. When there is a combination of errors and erasures, the 
decoder can correct up to $2 E+S<=n-k$ where $E$ is the number of erasures and $S$ the number of errors [7][20].

$\mathrm{Li}$ et al [20] introduce the network coding protocol that is based on RS codes. The protocol uses an opportunistic listening technique. Thus, the protocol depends on the topology and node density of the network. The authors claimed that the RS-NC technique achieves a $61 \%$ coding gain when compared to a non-coding approach, where the coding gain is the ratio of the number of transmission required by a non-coding approach to the number of transmissions used by the RS-NC technique to deliver the same series of packets to all nodes.

\subsubsection{Random Linear Network Coding (RLNC)}

The RLNC technique is another type of DNC techniques to encode and decode data packets. RLNC is an extended approach to the Linear Network Coding, where a node encodes a packet using linear transformations. When a group of intermediate nodes try to deliver information to a destination, each node generates a new packet which is a linear combination of received packets. The destination then performs a Gaussian Elimination to decode the independent received packets in a linear manner [8][9]. However, Ho et al. [11] have shown that the use of random linear codes is an efficient and practical technique to design linear codes to be used. In the RLNC, intermediate nodes independently and randomly choose the coefficients of the linear combination from a finite field called Galois Field (GF). To decode a packet, the receiver should receive an amount of linearly independent combinations of packets that equals to the number of source processes. 
The process of RLNC is divided into two operations [22]; encoding and decoding operations. As shown in Figure 2.9, the encoder is responsible to perform the encoding operation which combines linearly the original message with independently and randomly selected coefficients from GF. GF is a finite field with many elements. All operations presented in the finite field are caused by the elements within that field. As a result, GF is the most suitable field to be used in RLNC. Assume that a node has N data packets to send $P_{1}, P_{2} \ldots P_{N}$ simultaneously. Using RLNC and $G F\left(2^{s}\right)$, where $s$ is the size of the finite field, the node selects $N$ number of coefficients $c_{j}=c_{j 1}, c_{j 2} \ldots c_{j N}$ from the finite field forming a coding vector, and performs $\sum_{\imath=1}^{N} c_{j t} \times P_{\imath}$ to generate a new encoded packet $X$, that combines all data packets that the node wants to send. To illustrate using RLNC technique, a node has two data packets to send; $P_{1}$ and $P_{2}$. The node can send both data packets simultaneously by choosing two coefficients $\left(c_{1}, c_{2}\right)$ from the finite field and performing the previous linear operation which will result in an encoded packet that contains both $P_{1}$ and $P_{2} ; X_{1}=c_{11} P_{1}+c_{12} P_{2}$. The actual summation occurs in the form of matrix multiplication by forming the coding coefficients $c$, and the data packets $\left(P_{1}, P_{2} \ldots P_{N}\right)$ as matrices. Thus, the matrix form of $c_{\jmath l} \times P_{l}$ expression can be written as $X=C \times B$. 


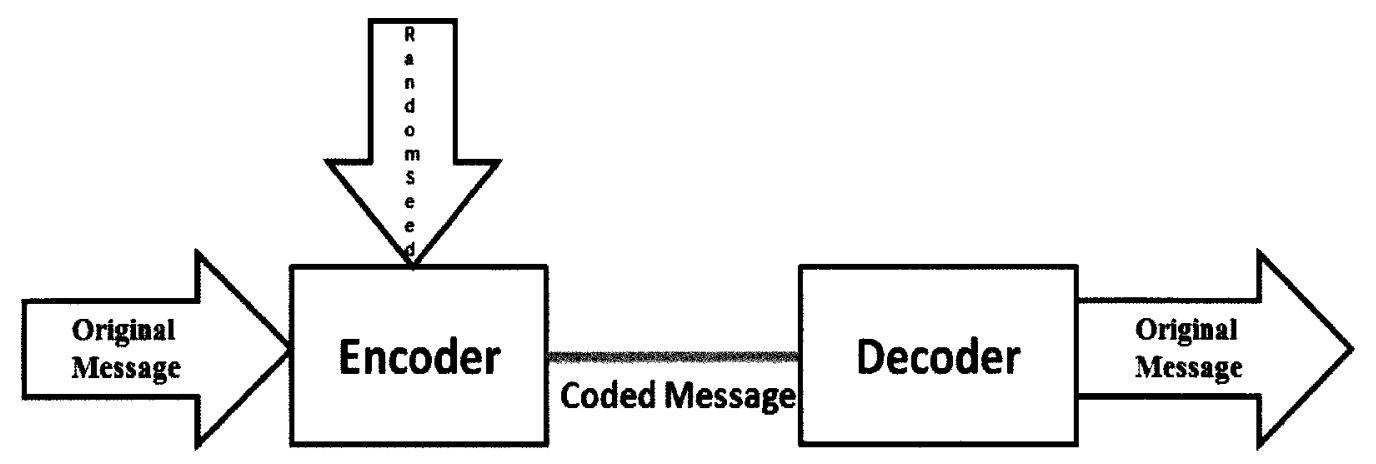

Figure 2.9: Random Linear Network Coding Diagram [22]

The construction form (Figure 2.10) of the new encoded packet $X_{J}$, which combines all data packets that the node wants to send, contains two parts; a header and a payload [22]. The header contains the coefficient vector (coding vector) which holds every coefficient that was chosen from GF and used to encode the data packets $\left(P_{1}, P_{2} \ldots P_{N}\right)$. Payload includes encoded data packets which are formed by the expression $\sum_{l=1}^{N} c_{l l} \times P_{l}$. The size of the new encoded packet $X$, is the summation of sizes of every coefficient in the header and sizes of the every encoded data packets in the payload.

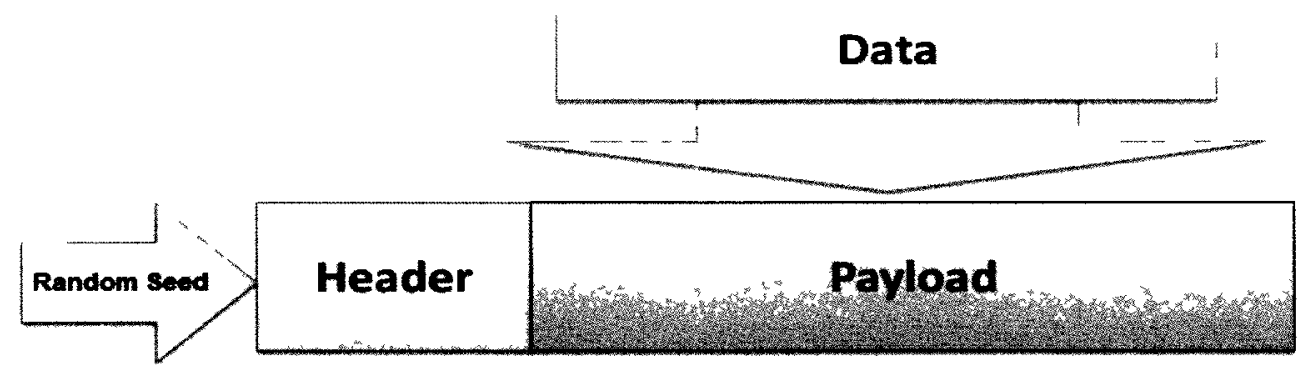

Figure 2.10: Encoded Packet Format [22]

The encoding procedure can also be referred to as the re-coding process under the condition in which encoding is proceeded in intermediate nodes in the network between 
several number of received encoded packets. However, the coding vector in the re-coding process is formed by arithmetic operations between the new coefficients which are chosen by the intermediate node from GF and the coefficients which were used to construct the received encoded packets. To illustrate, assume that an intermediate node received two packets $X_{1}=c_{11} P_{1}+c_{12} P_{2}$ and $X_{2}=c_{21} P_{1}+c_{22} P_{2}$. To perform the re-coding process the intermediate node chooses new coefficients $\left(d_{11}, d_{12}\right)$ from GF. After performing the re-coding process, the intermediate node constructs $X_{1}^{\prime}$ as followed;

$$
\begin{aligned}
& d_{11} X_{1}+d_{12} X_{2}=d_{11}\left(c_{11} P_{1}+c_{12} P_{2}\right)+d_{12}\left(c_{21} P_{1}+c_{22} P_{2}\right) \\
& =\left(d_{11} c_{11}\right) P_{1}+\left(d_{11} c_{12}\right) P_{2}+\left(d_{12} c_{21}\right) P_{1}+\left(d_{12} c_{22}\right) P_{2} \\
& =\left[\left(d_{11} c_{11}\right)+\left(d_{12} c_{21}\right)\right] P_{1}+\left[\left(d_{11} c_{12}\right)+\left(d_{12} c_{22}\right)\right] P_{2}
\end{aligned}
$$

where $\left[\left(d_{11} c_{11}\right)+\left(d_{12} c_{21}\right)\right]$ and $\left[\left(d_{11} c_{12}\right)+\left(d_{12} c_{22}\right)\right]$ are the new coefficients for the new encoded packet $X_{1}^{\prime}$.

As seen in Figure 2.9, the next step of the RLNC technique after the encoding process is the decoding process which is performed by the destination node after receiving encoded packets. The destination node applies a Gaussian Elimination method to decode linearly independent received packets. The destination node converts received packets into a matrix form and inserts the formed matrix row by row into its decoding matrix. The decoding matrix contains the destination's original packet and the encoded received packets. After that, the destination solves the linear system: $X=C^{-1} \times B$, where $B$ is the recovered data packets matrix, $C^{-1}$ is the inverse coefficient matrix and $X$ is the encoded received packet matrix. If the destination node receives an innovative packet which increases the rank of a decoding matrix, then the innovative packet will be inserted as the last row of the decoding matrix. On the other hand, if the destination node receives 
a non-innovative packet, then the innovative packet will be inserted and reduced to row of zeros by the Gaussian Elimination method and ignored.

\subsection{Benefits of Network Coding on Wireless Networks}

$\mathrm{NC}$ techniques can offer many benefits for wireless networks, such as increasing the overall throughput, decreasing the energy consumption of wireless devices, guaranteeing reliable transmissions, minimizing the delay, and etc [23][24].

Throughput and bandwidth: NC techniques can increase the overall network throughput by decreasing the number of transmission in the network over the time. NC decreases the number of transmission by combining several data packets into one encoded packet. In other words, NC techniques prevent wasting wireless networks bandwidth. [25][26][28][29] and [30] have showed the improvement of network throughput and bandwidth by using NC techniques.

Energy Consumption: wasting energy is considered one of the main issues in ad hoc wireless mesh networks. As shown in Figure 2.11, NC can solve energy consumption issues by combining several data packets into one encoded packet and send the new encoded packet in one transmission, unlike regular transmission where each data packet is sent separately and in a dedicated transmission which would waste more energy and drain quickly the battery life of wireless devices. [31] has discussed a scheme that reduces the energy consumption in wireless nodes. 


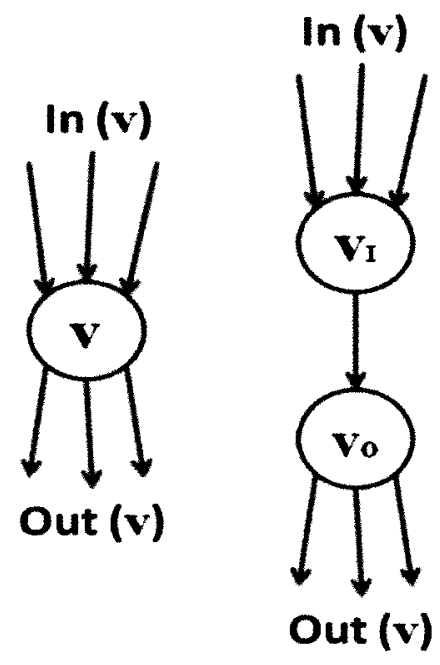

Figure 2.11: Regular Transmission vs. NC transmission

Transmissions Reliability: NC can ensure higher reliability mainly in the case of lossy networks, where Forward Error Correction (FEC) and Automatic Repeat reQuest (ARQ) algorithms do not perform well. In NC, the loss of one encoded packets does not essentially need to perform the retransmission mechanism to recover original data packets. The lost encoded packet can be received from any node which will allow the decoding process to be successfully preformed to recover all original data packets. Some schemes have been implemented in [32] and [33] which improve the network reliability. Based on this benefit, a paper was submitted in NetCod 2011 - The 2011 International Symposium on Network Coding Conference held in Beijing, China in July 2011. The title of the paper is "QoS and Protection of Wireless Relay Nodes Failure Using Network Coding".

Minimize Delay: Jain and Chou proved that NC can minimize the delay. As shown in Figure 2.12, the optimal multicast mechanism needs 3 extra time slots in order for all destinations to receive $a$ and $b$ data packets. On the other hand, the NC coding mechanism needs only 2 extra time slots for all destinations to receive $a$ and $b$ data 
packets. Some schemes have been discussed in [8][9][34] which reduce and minimize the delay by using NC.
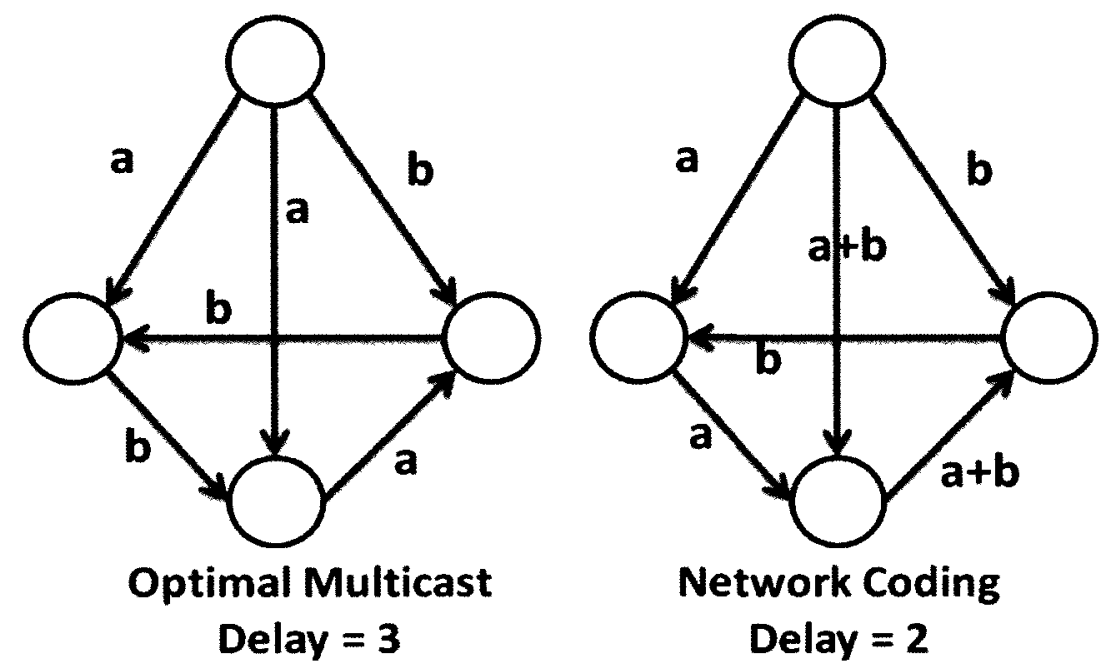

Figure 2.12: Delay [24]

Security: NC can reduce the complexity level by providing secured networks especially in RLNC. For example, RLNC provides a simple and a powerful way to develop security networks by encrypting only the source coefficients. This procedure can reduce the number of cryptographic operations necessary for a private transmission. [35] showed a scheme that increases the network security by using NC.

Chapter 3 will discuss more schemes, which have been theoretically evaluated or practically implemented in the literature, and show the benefits of $\mathrm{NC}$. 


\section{CHAPTER 3 - RELATED WORK ON MULTIMEDIA APPLICATION WITH NETWORK CODING AND NETWORK CODING BENEFITS}

This chapter discusses some related work that has been conducted using NC techniques for different multimedia applications. It also discusses some related work that shows the main benefits of the network coding techniques in improving the overall performance of wired and wireless networks.

\subsection{Multimedia and NC}

This section discusses some related work that has been conducted using NC techniques for different multimedia applications, such as Video Streaming.

\subsubsection{Peer-to-Peer Streaming With Hierarchical Network Coding}

In [25], the author studied a solution to prevent bandwidth consumption of internet resources using multicast protocols however; these multicast protocols have major limitations. The limitations lie within providing users the required bandwidth to support their traffic as it is too challenging for intermediate node to solve. Moreover, multicast protocols cannot send required packets to the receiver simultaneously. The author designed a solution by using the Video-on-Demand (VoD) technique which depends on unicast protocols. It is a client server solution and therefore this technique is not scalable. To solve the scalability problem, the author created the Content Delivery Network (CDN) which redirects users not to overload servers. The author also used Peerto-Peer (P2P) systems to dominate the bandwidth consumption. The author then supported the system with RLNC in sharing the content to increase the throughput by minimizing overheads but increasing the complexity of the system. However, he did not 
mention how the overhead would be minimized, neither shown any simulation result to prove the improvement impact of the design, but he mentions that he will evaluate the system performance by uplink sharing model.

\subsubsection{Network Coding for Peer-Assisted Multimedia Streaming}

In [26], the authors designed a solution for Peer-Assisted multimedia streaming using the NC technique to avoid several problems like increasing play back quality, initializing buffering delay, decreasing bandwidth consumption and scalability. The author supported Peer-Assisted streaming protocol with two techniques to prevent these problems; these are Random Push and RLNC. With the new proposed modification, the streaming content is divided into segments. Each segment holds a number of data blocks which contain the streaming content. Peers choose segments randomly and encode data blocks of chosen segments. This design has not been experimented but theoretically proves that it prevents the Peer Assisted multimedia streaming problems. Also, authors did not mention if they will testify their design and they did not mention the topology model that will be used to experiment the design.

\subsubsection{Network Coding for Wireless Video Communication}

In [27], the author described two scenarios for improving the capacity (Video Quality and Bandwidth) of wireless video communications. The first scenario is called NC-based Scheduler at a Wireless Base Station, where the author used the XOR technique to encode packets at base stations. The design successfully decreases bandwidth consumption but increases the delay which reduces video quality due to the waiting time in the queue at the receiver side which is not acceptable for video streaming. To solve this problem, the author decided to use Markova Decision Process with NC to 
improve the video quality. The second scenario that the author described is called $N C$ based Flow Mixing at a Wireless Base Station. The scenario uses Reed Solomon codes to mix different flows at the base station. This scenario has a high complexity level and sometimes sends non-desired packets to non-interested receivers. However, it performs better than the previous scenario in lossy channels.

The author in [27] has not shown any improvement of the capacity for wireless video communications. He was not able to provide simulation, experimental results or even a description of a model both scenarios by explaining a model to be used in their experiments.

\subsubsection{An Approach of Scalable MPEG-4 Video Bitstreams with Network Coding for P2P} Swarming System

The authors in [36] present an approach to increase the performance and scalability of the P2P swarming system. The authors supported the P2P swarming system with MPEG-4 fine granularity scalable (FGS) with NC technique. They mentioned the reason for not using the $\mathrm{NC}$ technique alone is due to the no scalability of NC protocols without providing evidence or referring to a previous work. However, he mentioned that $\mathrm{NC}$ can increase the availability of video by increasing the bandwidth resources, decreasing blocking scheduling problem of the P2P Swarming System (by allowing all nodes to produce encoded packets) and producing encoded blocks that are useful for all receivers. The MPEG-4-FGS coding uses two layer of bitstream to present the video sequences; base layer bitstream and enhancement layer bitstream. In this approach, they applied NC in base layer bitstream to provide high nodes availability. The enhancement layer helps to improve the quality of the video streaming. Thus, the overall performance 
of video streaming networks is increased. They proved the improvement impact of their approach by performing experimental simulation using NS-2. However, they did not mention the type of network coding they used in base layer bitstream and how they applied it in this layer.

\subsubsection{Minimizing Delay for Video Conference with Network Coding}

The paper [34] discussed a main issue of video conferencing which is the end to end network delay. Moreover, the authors highlighted another issue of video conferencing which is the high consumption of bandwidth. The paper discussed the benefit of network coding in minimizing the delay by providing an example shown in Figure 3.1.

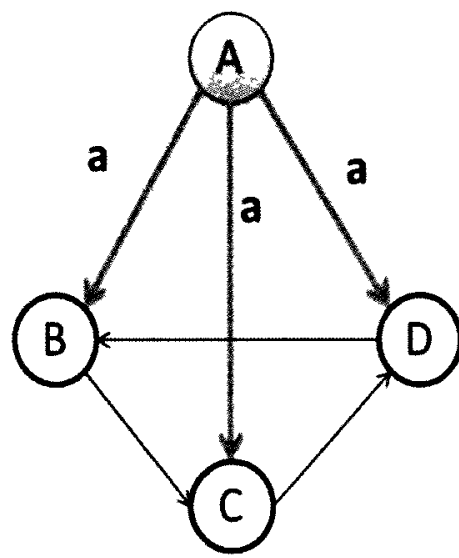

(a) Multicast

Throughput $=1$, Hop $=1$

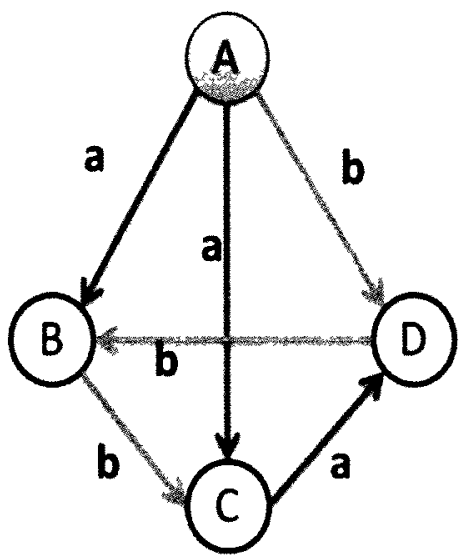

(b) Multicast

Throughput=2, Hop $=3$

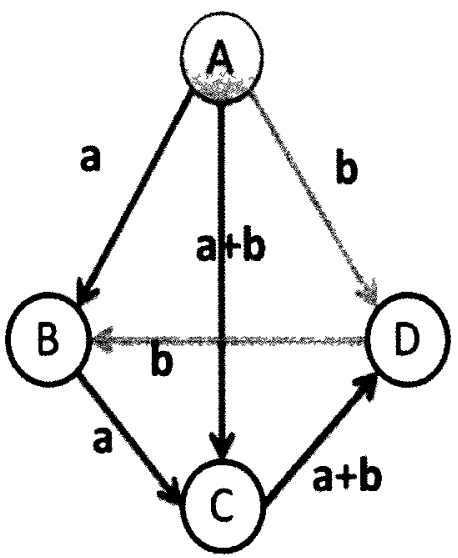

(c) Network Coding

Throughput=2, Hop $=2$

Figure 3.1: NC and Delay [34]

The main contribution of the paper is to compare XOR-NC with the multicast protocol to prove that XOR-NC could improve the performance of video conferencing by minimizing the delay under the same throughput achievement. The authors proved their contribution by providing simulation results which were based on the number of receivers 
with the throughput remaining as a static factor using PlanetLab testbed. Also, they only considered static video conferencing networks.

\subsubsection{Priory Ordering \& Packetization for Scalable Video Multicast with Network} Coding

The authors in [37] proposed a protocol to improve the robustness and flexibility of video multicasting mechanism over IP networks using RLNC. The proposed model is divided into three parts; source which performs scalable video coding (SVC) and packetization, relay which combines packets that are sent by the source using RLNC and forward the encoded packets to the destination, and destination which has the ability to solve the received combination of the encoded packet and recover the original messages. They compared the proposed model with SNR based and default SVC methods. The results proved that the proposed method improves the overall performance of the network. The results were based on H.264 video traffic. The authors tested standard sequences of mother\&daughter, foreman and football sequence of size $352 \times 288,30$ frames per second and 300 frames in total.

\subsubsection{Secure Network Coding for Multi-Resolution Wireless Video Streaming}

The paper [35] introduced an RLNC protocol called Secure Practical Network Coding (SPOC). The main purpose of modeling SPOC was to serve privately wireless users who have different subscription quality with video streaming. The authors determined that the quality of videos using the regular broadcast scenario could be corrupted due to high packet loss. They also determined that the wireless network environment has security issues due to the overhearing mechanism. To demonstrate the problem, they used the topology shown in Figure 3.2. In Figure 3.2, a source S streams 
video to destinations; $\mathrm{A}, \mathrm{B}$ and $\mathrm{C}$ through relay nodes $\mathrm{R} 1, \mathrm{R} 2$ and $\mathrm{R} 3$ wirelessly. The destinations subscribed for special video quality service, therefore a mechanism must be developed to guarantee secured and a reliable transmission over the wireless medium.

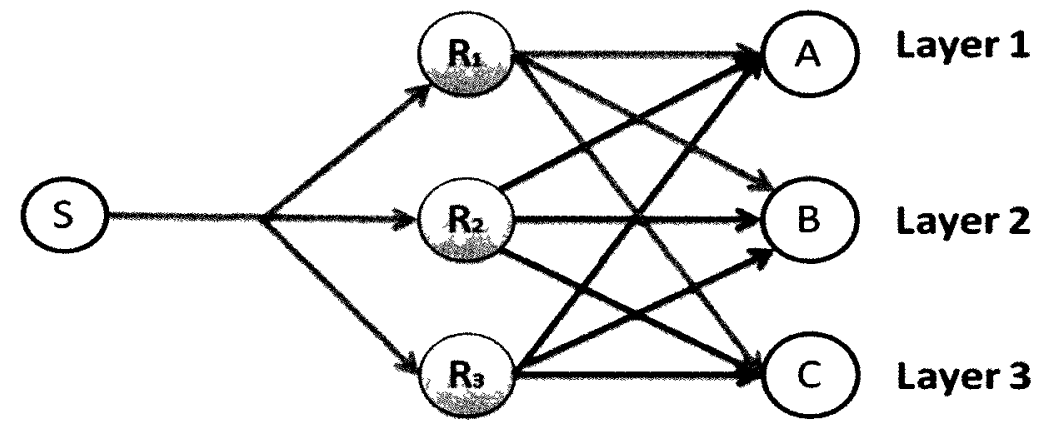

Figure 3.2: SPOC Topology

Figure 3.3 shows SPOC protocol processes; the source sends the video to a multilayer video process where video is divided into several layers. All video layers will be sent to a network encoder to perform the RLNC technique and produce one encoded packet including all video layers. Before the encoded packet is transmitted, an SPOC encrypts source coefficients are used.

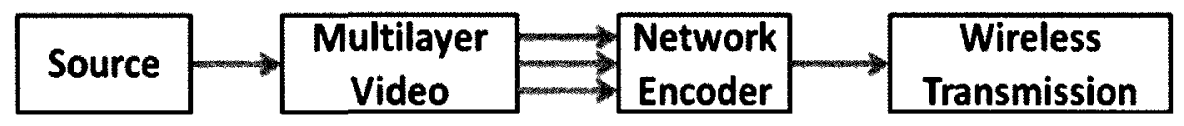

Figure 3.3: SPOC

The authors used ns-2 to compare the proposed protocol with two other protocols; SPOC without Multilayering process and SPOC without RLNC. They proved that the proposed protocol improves the security level in the Multi-Resolution wireless video streaming networks from an eavesdropper attacks. However, they did not consider mobility scenarios in their simulation where eavesdropper is more active. 


\subsubsection{Experimental Measurement for VoIP with Network coding in IEEE 802.11}

The paper [28] shows an implementation of a design based on Voice over IP (VoIP) communication under multicast IEEE 802.11 networks. The main contribution of the paper is to increase the performance and QoS with current coast of VoIP communications. The authors used Session Initiation Protocol (SIP) with NC; where SIP Server is equipped with an encoder which combines linearly two received messages by the two nodes who are communicating with each other over IP. Figure 3.4 shows the procedure of establishing, exchanging and ending a call between two nodes.

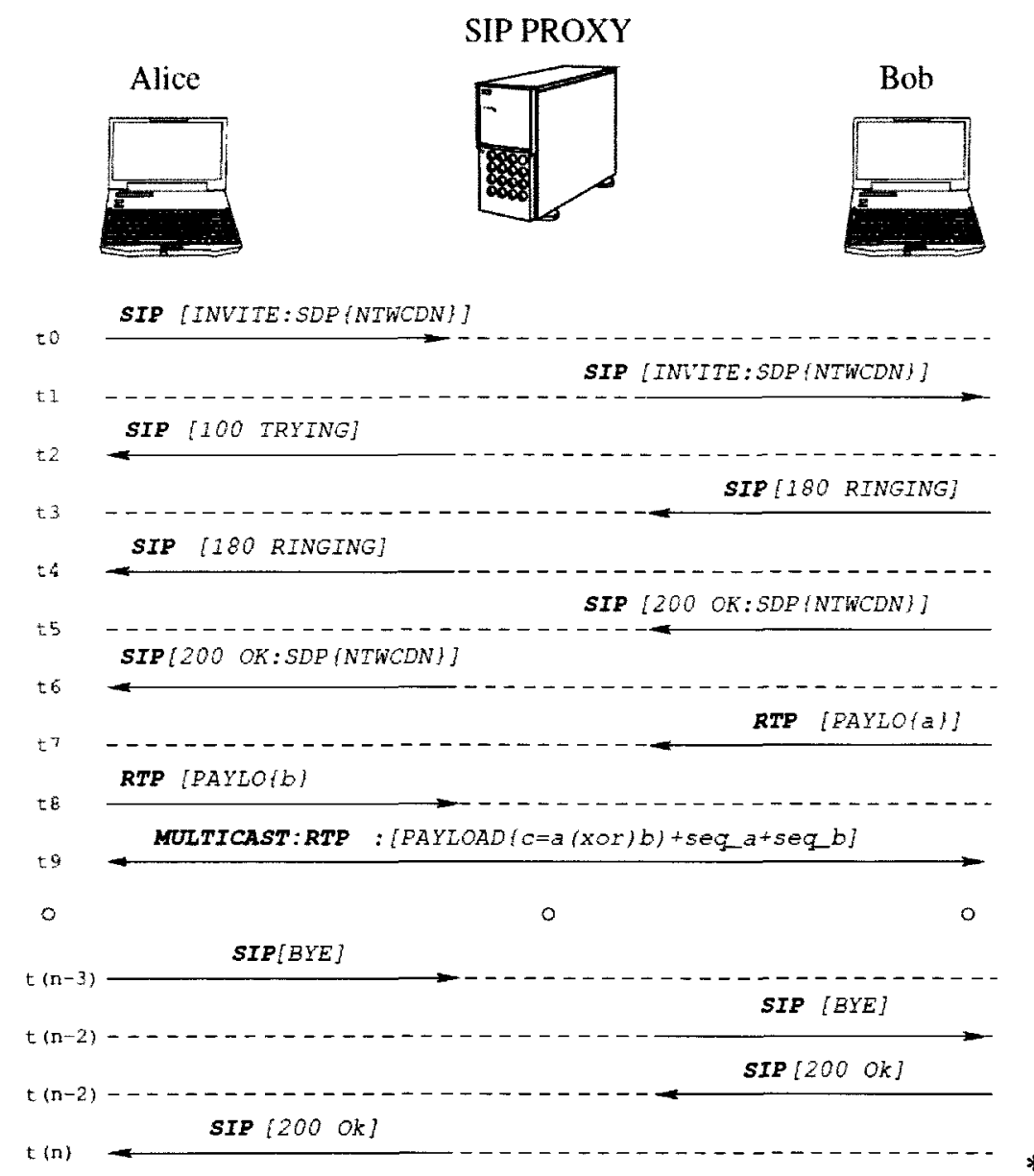

Figure 3.4: SIP with NC 
The authors compared the proposed protocol with the traditional VoIP phone calls protocol. They showed in their result that the proposed protocol reaches higher QoS than the traditional protocol by maximizing the SIP server downlink throughput. On the other hand, the results showed that the proposed protocol capacity is 6 calls (pairs of node) to gain higher QoS. Also, their paper does not consider a conference call scenarios or mobility scenarios.

\subsubsection{CODECAST: A NETWORK-CODING-BASED AD HOC MULTICAST PROTOCOL}

This paper [29] introduced a multicast RLNC protocol called CodeCast which is used for video surveillance. The protocol basically collects transmitted video images from different cameras that monitor industrial environment. The protocol also tries to solve delay and packet delivery ratio for a single source multi-receivers networks.

The application generates a different number of packets which have the same size, and encodes them into one encoded packet. The authors gave the name "blocks" to the encoded packets. The block is a number of packets to be encoded in the block and contains two main parameter; blockid and blocksize. The blocksize varies from block to block depending on the delay constraints which are calculated from the frame generation rate of the applications. The receiver nodes have a timer called blocktimer which is used by these nodes to start decoding and recovering blocks when all packets of a block are received.

As mentioned in the literature, nodes which use RLNC should be able to decode the block even if the number of received packets is smaller than the blocksize. The way this can be done is by performing partial decoding of the received packets. The protocol is not considering this type of decoding and the paper does not talk about it. 
The authors showed that end-to-end delay increases by increasing the blocksize as more frames are needed to perform the encoding operation, but the authors only presented their result using two block sizes 4 and 8 . Moreover, they have shown that by increasing the blocksize, the network throughput will increase. They compared the proposed protocol with the On Demand Multicast Routing Protocol (ODMRP) with a small overhead using QualNet. Many literatures have claimed that ODMRP is one of the best multicast routing protocols in mobile networking. However, the results showed that CodeCast perform better than ODMRP regardless of blocksize, node speed or drop probability.

3.1.10 Reliable Wireless Broadcast with Random Network Coding for Real-time Applications

The authors of [32] introduced a multisource wireless broadcast protocol based on RLNC. The main goal of the proposed protocol is to be used in multi-player video gaming, especially in wireless broadcast networks called Network Coded Piggy-Back (NCPB). The procedure of the proposed protocol is;

- Initialization: A number of nodes which would like to join the game negotiate with each other to obtain IDs and exchange coding vector that is used during the game session.

- Encoding/transmission: done as discussed in the literature.

- Receiving /decoding: done as discussed in the literature.

However, the authors did not talk about a way of creating and generating generations at the source node. The authors used IEEE 802.11 broadcast, Piggy-Back Retransmission (PBR) and Multi Point Relay (MPR) Network Coded Piggy-Back 
(NCPB) to compare the proposed protocol with in terms of packet delivery ratio (PDR) and delay using QualNet simulator. The results showed that both the PDR and delay are suitable for gaming environment and better than other protocols regardless of the number of nodes which are joining the game session.

\subsection{The Benefits of Network Coding}

This section discusses some related work that has been implemented in the literature on the main benefits of $\mathrm{NC}$ techniques.

\subsubsection{A Proactive Network Coding Strategy for Pervasive Wireless Networking}

The paper [8] introduced a broadcast proactive protocol which solves various problems for reactive NC protocols, which in return solves the problem of wireless networks in distrusting efficiently information across the network. The problems of reactive $\mathrm{NC}$ include;

- A new combination of a packet is only generated when the received packets are innovated.

- Receiving innovation packets in one area at the same time may cause all nodes in the same area to generate and send encoded packets at the same time. Thus, the probability of collision in the network increases.

- The dependency on forward factor which depends on the number of node neighbors. It is a factor that allows a node to send a new encoded packet all over the network.

Basically, in the proposed protocol mechanism a node sends an encoded packet when it receives a number of innovated packets, otherwise the node remains inactive by activating the stopping condition mechanism. There are two type of stopping conditions 
which specify the type of proactive NC protocol; weak and strong stopping conditions. A stopping condition protocol allows the source and the receivers to exchange messages which inform the source if receivers have successfully encoded its packets so that the source can turn into an inactive mode. The difference between weak and strong stopping conditions is that the overheads of encoded packets in weak stopping condition are smaller compared to strong stopping condition. The difference between the two conditions mainly exists due to the fact that nodes in weak stopping condition do not need to know all traffic information of all nodes in the network, where in strong stopping condition nodes should collect enough information about their neighbors by assuming the exchanged data traffic between the nodes. The authors compared both protocols; weak and strong proactive $\mathrm{NC}$, in terms of delay, PDR and overhead size with different transmission rates using NS-2. Also, they compared proactive NC with reactive probabilistic network coding. The authors states that weak and strong proactive NC can successfully be used in actual networks. They also stated that proactive NC performs better in large networks by having a smaller overhead and a shorter delay. However, authors did not consider mobility scenarios in their study.

\subsubsection{AN Efficient Operation of Wireless Packet Networks Using Network Coding}

The paper [31] discussed a theoretical proof of the improvement that RLNC can cause for lossy and lossless wireless networks using the butterfly topology. They showed the benefit of using $\mathrm{NC}$ in improving the performance of wireless networks and saving the nodes' energy. The paper showed that there is a gain improvement in lossless wireless networks only in case of multi-source networks. However, NC showed promising gain improvement in lossy networks in the case of single and multiple source networks. 


\subsubsection{On the Scaling Law of Network Coding Gains in Wireless Networks}

The paper [38] discussed a major wireless network factor which is Delay. Delay is a major factor in many applications in the real world, such as Voice and Video. The paper studied the effect of RLNC on two types of networks, elastic and inelastic. The authors define elastic networks as a network where users get services without delay constraints, where inelastic networks are a network with delay constraints and users prefer not to receive a service if the delay exceeds the upper bound constraint.

The analysis showed that NC minimizes the delay for both types of networks in the case of a single hop scenario and assumes a Poisson arrival only differs as following;

- Elastic networks: the performance of elastic networks improves as the file size increases.

- Inelastic networks: number of receivers in inelastic networks increases under the same delay constraints. In other words, the throughput of inelastic networks increases.

Furthermore, the paper tried to generalize the study to multiple hop networks by grouping nodes into layers, while assuming that there is no communication between nodes at the same layer in the networks and nodes are not allowed to forward packets unless all nodes in the layer have received the packets.

The authors did not explain how the layered topology should be created and how broadcasting or multicasting packets could be specified to the required layer, so all nodes in the layer can receive and forward the packets. Also, their model is not a practical solution in cases of mobility where layering will not be a good solution. 


\subsubsection{Network Coding Performance for Reliable Multicast}

The paper [33] studied the benefits of NC for wireless networks compared to the traditional error control techniques, such as ARQ and rate-less coding. The authors preformed this study based on a tree-based reliable multicast networks and specified the study on the end-to-end ARQ, rate-less coding, link-by-link ARQ and NC technique. The paper showed a positive effect of $\mathrm{NC}$ over end-to-end ARQ. In other words, $\mathrm{NC}$ reduces the number of retransmission in a network. Similarly, rate-less coding and link-by-link ARQ have shown almost the same improvement. However, when the authors preformed the experiments to test the end-to-end delay, they assumed that the networks have reliable transmissions for acknowledgments which are used in NC to inform the source that the receiver have successfully decoded and recovered the original information. They also assumed that the number of children of each node is constant, which is not a practical assumption especially when the nodes are in a mobile environment.

\subsubsection{On the Performance of Random Linear Network Coding in Relay Networks}

The authors in [39] studied the reliability performance of RLNC in a singlesource-single-receiver unicast relay network. They compared the reliability performances of two ARQ schemes; Enhanced ARQ (ARQ-E) and Single Path Routing ARQ (ARQSPR) based on complexity and overhead. They defined the reliability gain as the expected number of channels used per data bit received at the receiver. The experimental result showed ARQ-E, which is a high complex protocol in terms of scheduling, has almost the same reliability performance of RLNC. On the other hand, ARQ-SPR could reach the same level of reliability performance when the overhead per transmitted packet is negligible. However, the authors in this study only considered a single-source-single 
receiver scenario which is not a practical consideration in the real world where there are of multiple number transmitters and receivers.

\subsubsection{Wireless Network Coding with Improved Opportunistic Listening}

The paper [30] introduced a new usage for NC which improves the throughput of wireless networks. The paper discussed the use of NC over carrier sense multiple access (CSMA) schemes. The authors provided a new algorithm based on IEEE 802.11 MAC protocol and called it (NC-MAC). Basically, the new algorithm takes the advantages of the wireless environments to improve the network performance. In other words, the proposed algorithm allows nodes to decode overheard transmitted packets; acknowledgments or data, so that all the nodes have a full knowledge of the correct coding opportunities. The authors compared the proposed algorithm with regular NC algorithm. Their results have shown that the proposed algorithm has better network performance in terms of throughput when compared with the regular NC algorithm. However, they used static topologies in the simulations so the results do not demonstrate real life implications. Also, they used XOR-NC which depends on previous knowledge of information in order to decode encoded received packets.

\subsubsection{Broadcasting in Multihop Mobile Tactical Networks: To Network Code or Not}

The paper [7] provided a comparison study between the different types of broadcast protocols that are widely used in wireless networks to discover a suitable protocol for multi-hop tactical networks. The authors selected three broadcast protocols for their studies; flooding, Simplified Multicast Forwarding (SMF) and Partial Dominant Pruning (PDP). They also implemented XOR and Reed Solomon NC techniques with SMF and PDP. The authors showed that adding NC to SMF and PDP for multi-hop 
tactical networks improves the performance of the networks in terms of PDR. However, this resulted in an issue where $\mathrm{NC}$ increases the latency of delivering and decoding packets. Further explanation will be discussed in chapter 4 about the SMF and PDP as they will be used in the thesis.

\subsubsection{On MAC Scheduling and Packet Combination Strategies for Practical Random}

Network

The paper [9] introduced RLNC technique and showed the technique's benefits over broadcast wireless networks. The authors implemented RLNC based on CSMA to show the MAC layer impacts on NC performance. They used IEEE $802.11 \mathrm{~b}$, IEEE $802.11 \mathrm{~b}$ with pseudo broadcast, IEEE 802.11 with pseudo broadcast and RTS/CTS and Ideal MAC with the proposed algorithm to perform their study. They compared all four protocols in terms of PDR, delay, collision ratio and overhead size, and showed the positive impact of $\mathrm{NC}$ on the network performance. However, they did not consider mobility scenarios in their studies. Furthermore, the RLNC implementation will be discussed more in Chapter 4 of thesis.

\subsection{Discussion and analysis}

As discussed previously and from the analytical or practical works that have been conducted, NC is a promising and a potential technique to be used in several applications, especially multimedia applications, such as video and voice. Also, the literature has shown that NC has the potential to increase wireless network capacity. Based on the previous discussed literature, the thesis has made a comparison between the different broadcast protocols over static and mobile wireless networks based on the multimedia 
traffic. The following highlights the important points of network coding in the area of multimedia applications and $\mathrm{NC}$ benefits based on the literature review:

- Broadcast protocols are suitable solutions to prevent bandwidth consumption of the internet resources, where bandwidth consumption is a major issue in wireless networks and multimedia applications. The literature shows that the benefits thought of using multicast protocols to solve the problem.

- The previous works have theoretically shown that RLNC improves the network throughput and saves nodes' energy in lossly network for both one source and multiple source scenarios. As in real life, the lossly networks are more active.

- The delay is a major issue in multimedia applications. The survey showed that RLNC improves the performance of the network by minimizing the delay.

- The survey showed that RLNC has almost the same performance of ARQ-SPR with small overhead, ARQ-E, rate-less coding and link-by-link ARQ in static and low density networks in terms of reliability.

- Previous studies proved theoretically that implementing NC on top of MAC layer protocols improves the throughput of static wireless networks.

- The survey showed implementing some NC techniques, such as reed Solomon and XOR on top of optimized broadcast protocol improves the PDR of multi-hop tactical networks. In [7], they used SMF and PDP as optimized broadcast protocols in wireless networks.

- Another study in [9] suggest that implementing RLNC on top of IEEE $802.11 \mathrm{~b}$ shows promising results for multimedia applications to improve PDR, delay, collision ratio and overhead size for low density static wireless networks. 
- Many proposed unicast algorithms have been theoretically studied building up RLNC on top of multimedia transfer technique. [25] has showed a unicast protocol using RLNC on top of several multimedia techniques, such as CDN to minimize the overhead of the transmitted packet which decreases the bandwidth consumption. [26] has showed the implemented RLNC with Random Push on top of Peer-Assisted streaming protocol. [7] uses RS and XOR-NC in base stations with single or multiple types of multimedia traffic. [28] shows an integration study between NC and VoIP protocol. The integration was between the NC technique and SIP which shows improvement in QoS for voice traffic.

- Scalability and throughput are the main issues in wireless multimedia networks. XOR-NC shows promising results in implementing NC with P2P swarming system which is used to transmit MPEG-4 video traffic. CodeCast is an example of an implemented protocol using RLNC and shows an improvement in terms of throughput in video surveillance network.

- As mentioned before, delay is the major issue in multimedia applications and XOR-NC has shown a high performance in minimizing the delay with video conference networks. RLNC is also seen in multi-player video gaming, especially in wireless broadcast networks (NCPB) and shows an improvement in PDR and end-to-end delay.

- Robustness and flexibility are the main issues in the video multicast techniques. RLNC has provided a better network performance in terms of scalability and flexibility with H.264 video traffic. 
- RLNC has proved more promising results in security and multimedia application quality RLNC, as SPOC was implemented in [35]. RLNC improves the security level in Multi-Resolution wireless video streaming networks from an eavesdropper attack.

- Most of the proposed or implemented algorithms in multimedia wireless networks are either related to unicast or multicast scenarios. In other words, there is not much work done on studying or implementing algorithms on broadcast scenario over wireless networks, especially wireless multimedia networks.

- Most of the experiments and the results were based on static networks. However, mobile networks are the actual representation of the real life scenarios and networks; where nodes move all around.

Based on the previous highlights, the simulations in this thesis will be performed using schemes from [7] and [9] using multimedia traffic with ad hoc wireless mesh networks. The simulations results will be compared in terms of PDR, latency and jitter. 


\section{CHAPTER 4 - PROTOCOLS AND WIRELESS MODEL UNDER STUDY}

This chapter discusses the concept of SMF, PDP and RLNC protocols which are studied in the thesis. SMF and PDP are considered as one of the most optimized broadcast protocols for Mobile Ad-hoc Networks (MANETs) and wireless mesh networks (WMNs) [7]. However, RLNC is a new promising paradigm to improve the performance of wireless networks [8][9]. The chapter also describes the network topology and the mobility model which are used in the thesis.

\subsection{Simplified Multicast Forwarding (SMF) Protocol}

SMF Protocol is an optimized multicast/broadcast protocol which provides a forwarding plan for packets from source to destination. The SMF protocol uses the Duplicate Packet Detection (DPD) mechanism to maintain multicast/broadcast forwarding packets. The key benefit of SMF protocol is the improvement of the performance of relays in dynamic networks [40].

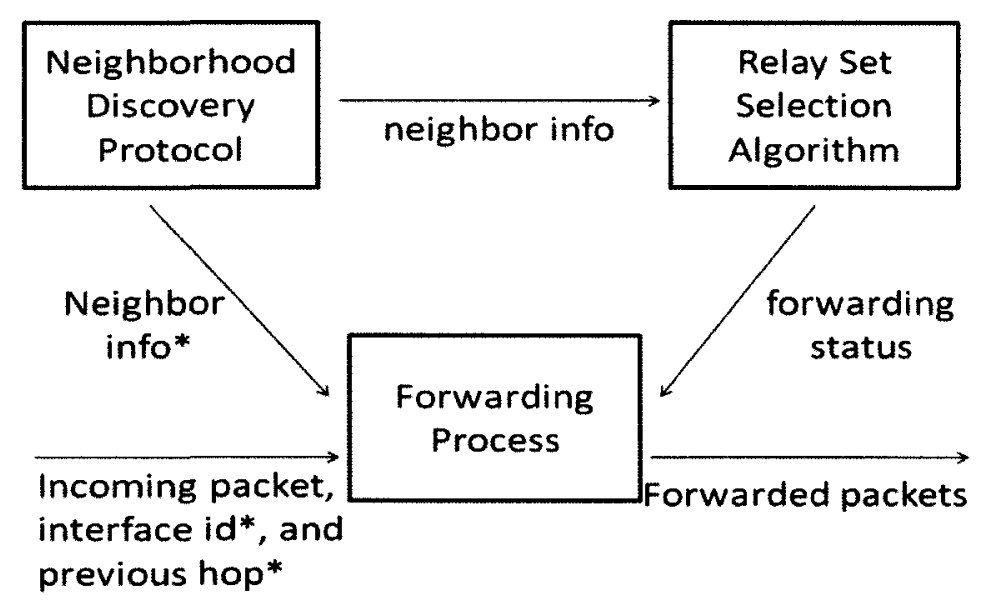

Figure 4.1: SMF Components

As shown in Figure 4.1, SMF protocol consists of three main components: 
1. Relay Set Selection: the input of this component is the neighbors' information which is collected by the Neighborhood Discover protocol. Neighbors' information demonstrates the relays' statuses which are important for the Forwarding Process in the network

2. Neighborhood Discovery: this component is a protocol to discover and collect all information about the nodes in the network. Neighborhood Discovery protocol performs two-hop neighborhood discovery. This information is used by the other two components of SMF protocol.

3. Forwarding Process: this component is responsible for forwarding packets based on the neighbors' information, forwarding status and other information that is contained in the incoming packet header.

Basically, the SMF node uses HELLO messages to collect information about the 2-hop neighbors in the network. Based on the collected information, SMF node selects a subset of the 1-hop Multi-Point Relay (MPR) nodes. Finally, the node broadcasts packets to this subset which floods received packets to all 2-hop nodes. The selection of the subset and forwarding the packets in SMF protocol are based on the collected information and stored information in the incoming packet header.

Broadcast protocols usually have a high ratio of received duplicated packets at the destination side. Due to the wireless medium characteristics, packets may get lost in the medium which causes a retransmission mechanism to occur for recovering these packets (which also increases the ratio of duplicated packets to be received at the destination). Thus, SMF has a duplicated packet detection mechanism to avoid receiving any repetitive 
packets. This mechanism uses IP address Identification "ID" field to detect any duplication where each packet has a unique "ID" field [7][40].

In the current thesis, three different versions of SMF protocol are used:

1. SMF without NC: un-modified SMF protocol

2. SMF-XOR: SMF protocol with the XOR-NC technique which is explained in 2.2.2.1

3. SMF-RS: SMF protocol with the RS-NC technique which is explained in 2.2 .2

\subsection{Partial Dominant Pruning (PDP) Protocol}

Dominant Pruning (DP) is a broadcast protocol which can minimize the number of un-needed transmissions. Similar to the case of SMF, DP uses HELLO messages to collect information about 2-hop neighbors in the network. Based on the collected information and the PDP algorithm, the source node selects a set of nodes to broadcast the sent packet without the need for all source's neighbors. PDP algorithm is considered as one of the optimized broadcast protocol for MANETs and wireless mesh networks.

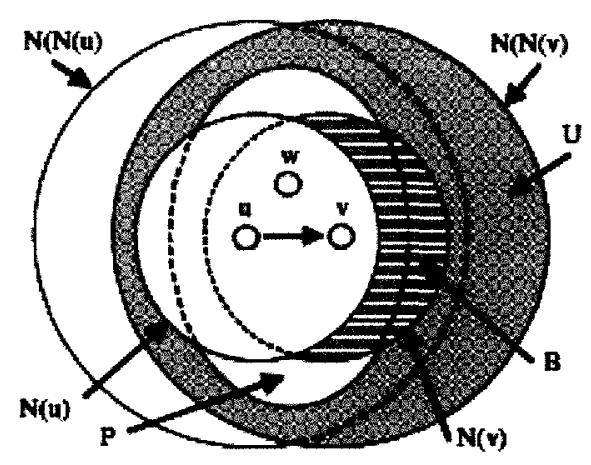

Figure 4.2: PDP Protocol [41] 
Assuming that $G=(V, E)$ where $G$ is a simple graph, that represents wireless network, contains $\mathrm{V}$ number of nodes and $\mathrm{E}$ number of links between the wireless nodes. Assume that, $N(u)$ is the list of neighbors of node " $u$ " and $N(N(u))$ is the list of 2-hops away neighbors of "u". As shown in Figure 4.2, $\{u\} \subseteq N(u) \subseteq N(N(u))$ and if $u \in N(v)$ then $N(u) \subseteq N(N(v))$ where $\mathrm{v}$ is a node in the network. Also assume that " $u$ " and " $v$ " are neighbors where " $u$ " is the source and " $v$ " is the receiver. In PDP algorithm, the source broadcasts packets without providing information about its neighbors. As a result, the destination cannot separate $N(N(u))$ from $N(N(v))$. PDP, besides excluding $N(u)$ and $N(v)$, can exclude $N(N(u) \cap N(v))$ nodes from $\mathrm{N}(\mathrm{N}(\mathrm{v}))$ so the destination " $v$ " uses the 2hop away neighbors $N(N(v))-N(u)-N(v)-N(v) \cap N(N(u))$ as the forwarding nodes of the broadcasted packets from the source " $u$ ". Figure 4.3 shows the PDP algorithm where $\mathrm{F}$ is the forward node list size [7][41].

1. Node $\boldsymbol{V}$ uses $N(N(u)), N(u)$ and $N(v)$ to obtain

$$
\begin{aligned}
& \qquad \begin{aligned}
P & =N(N(u) \cap N(v)), \\
U & =N(N(v))-N(u)-N(v)-P,
\end{aligned} \text { and } \\
& B=N(v)-N(u)
\end{aligned}
$$

2. Node $\boldsymbol{V}$ then calls the selection process to determine $F$.

\section{Figure 4.3: PDP Algorithm [41]}

In this thesis we used three different version of PDP protocol;

1. PDP without NC: un-modified PDP protocol

2. PDP-XOR: PDP protocol with the XOR-NC technique which is explained in 2.2 .2 .1 
3. PDP-RS: PDP protocol with the RS-NC technique which is explained in 2.2 .2

\subsection{Random Linear Network Coding Protocol}

The RLNC concept was discussed in 2.2.2.3. This sub-section discusses the MAC layer Protocol which is implemented with RLNC technique [8][9].

The protocol is built on top of the MAC layer protocol based on Carrier Sense Multiple Access (CSMA) which is affected by collisions, interference, and random scheduling of the packets which is suitable for wireless networks. The MAC layer protocol (used in this thesis) is based on broadcast transmissions. The protocol does not use any acknowledge mechanism where acknowledgement is not needed in most of the multimedia streaming transmissions. Moreover, the MAC layer protocol used has the same specifications of IEEE $802.1 \mathrm{lb}$ where maximum data rate is $11 \mathrm{Mbit} / \mathrm{s}$ using frequency of $2.5 \mathrm{GHz}$. However, IEEE $802.11 \mathrm{~b}$ cannot support larger packet size and high data rate, such as HDTV and SDTV traffic. As a result, I increased the data rate from $11 \mathrm{Mbit} / \mathrm{s}$ to $24 \mathrm{Mbit} / \mathrm{s}$ was increased in this thesis so that several types of multimedia traffic can be supported.

The MAC protocol is also supported by the RLNC technique to increase the performance of wireless networks in terms of delay and PDR. RLNC is designed with a parameter called Forwarding Factor which is the ratio between the number of packets transmitted and the number of innovative packet received at each node. In other words, it is the average number of packets that every node in the network can broadcast. The factor essentially helps the nodes to make the decision of creating a new random combination if the node receives an innovation packet. For example, if the value of the Forwarding 
Factor is 1 , then the node waits for one innovative packet to create a random combination of packets.

There is another factor is considered in the RLNC technique which is used in the MAC protocol. This factor is the NC Timer (T). The NC Timer gets activated for each innovation packet that is received at each node in the network. When the NC timer is expired, the node makes a decision to create then broadcast the new encoded packet. NC timer is a uniform random variable in [0, Tmax]. The main advantage of the NC Timer is that it enhances the progress of delivering encoded packets by reducing the noninnovative packets transmissions. Thus, the number of transmissions will be reduced. The reduction of the number of transmissions and random choice of NC timer value reduces the probability of collisions in the MAC layer. The only disadvantage of the NC timer is that it increases the overall delay of the network. As a result, the choice of NC timer value shall be mainly considered based on the performance of the network. [9] selects $\mathrm{T}=$ $20 \mathrm{~ms}$ as a value for the $\mathrm{NC}$ timer.

\subsection{Network Topology}

The multimedia network in this thesis utilizes ad hoc wireless mesh networks. A $\mathrm{WMN}$ is a special type of ad hoc network. A WMN is a decentralized, dynamic selforganized and self-configured network where nodes can establish connections with other nodes. Nodes in an ad hoc wireless mesh network are a mix of source, relay, and destination. In other words, each node can be a source, a receiver, and also can act as a relay to forward packets to nodes that are not directly connected to the source [42][48].

Figure 4.4 shows two types of mesh networks; Infrastructural Mesh Network and Client WMNs. The Infrastructural Mesh Network simply connects all routers (Base 
Stations/Sources) in the networks. Client WMNs basically connects a set of clients (receivers) together. The mix between both types of mesh networks is called Hybrid WMN which is used in our thesis.

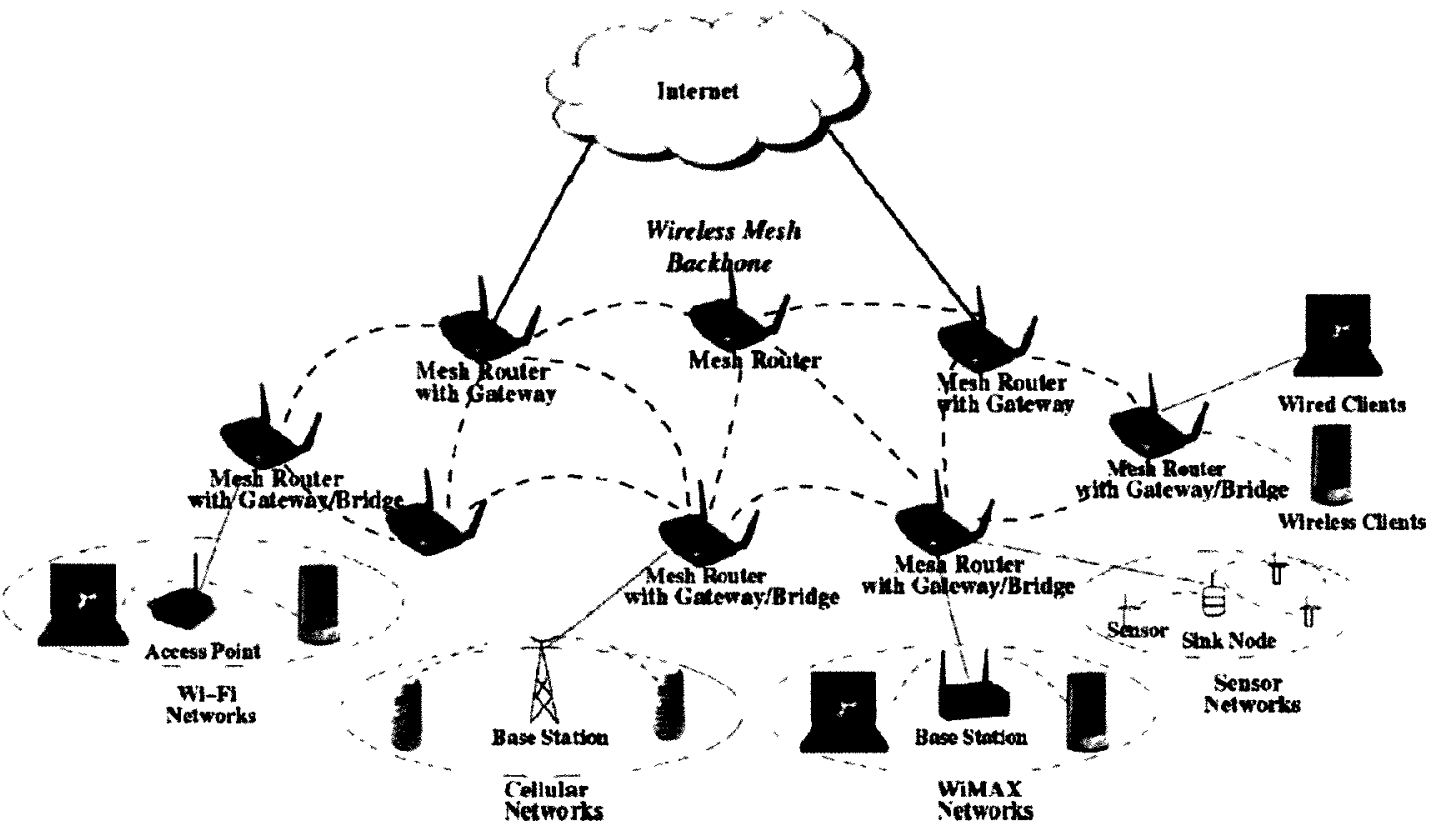

Figure 4.4: Wireless Mesh Network [42]

The reasons for using ad hoc wireless mesh networks to establish multimedia broadcast communications in our thesis are:

1. The network supports multi-hop mechanism.

2. The network has self-configuring characteristics.

3. The network can be easily integrated with existing wireless networks.

4. The network supports mobility.

5. The network supports peer-to-peer communication and the access to the internet. An ad hoc wireless mesh network can be considered as a MANET by converting the static characteristics of the nodes to dynamic characteristics. In other words, a 
WMN can be a MANET when a mobility model is added to the network. A commonly used mobility model that reflects real world mobility scenarios is the Random Waypoint mobility model. Random Waypoint mobility model was used in our study to testify the impact of mobility on NC protocols [43][48].

\subsection{Mobility Model}

Johnson and Maltz [44] were the first to propose the Random Waypoint Mobility model. Figure 4.5 shows an example of the movement of a wireless node using the Random Waypoint Mobility model. The Random Waypoint Mobility model became the most popular model in evaluating MANET and showing the impact of mobility on the different types of routing protocols because it is simple to use and widely available. Thus, Random Waypoint is used in our thesis to study the impacts of mobility on NC. In Random Waypoint model, the mobile nodes are moving randomly and without any boundaries; different parameters, such as direction and speed of nodes are chosen randomly [44].

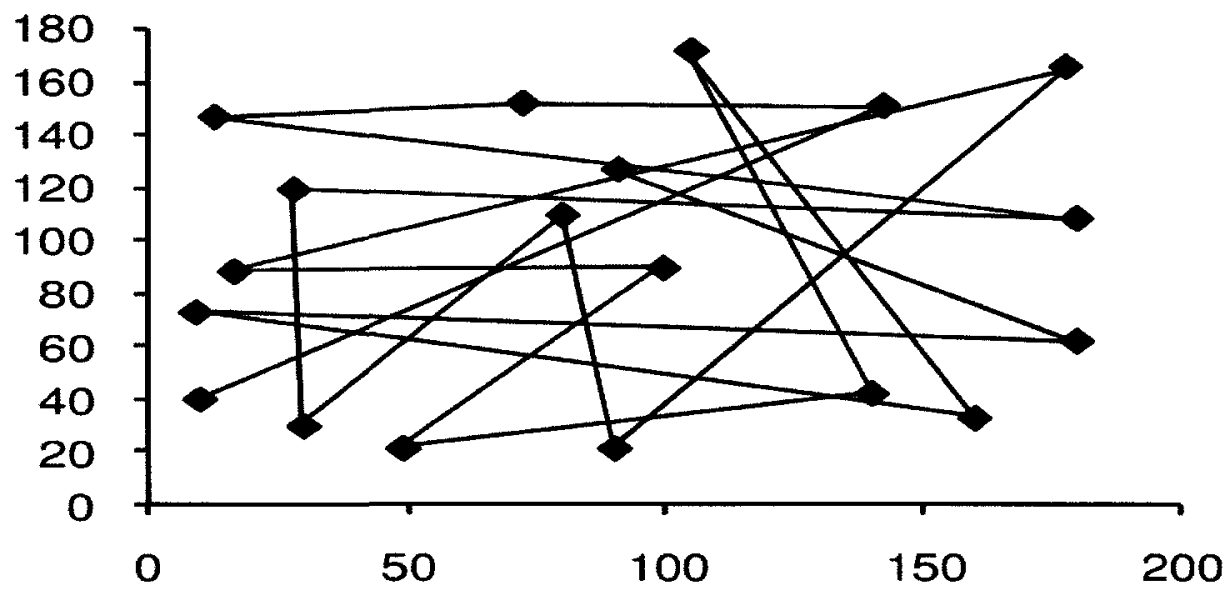

Figure 4.5: Random Waypoint Mobility Model [44] 
The Random Waypoint Model uses pause times for changing between speeds and direction of the mobile node. For example, the mobile node starts the scenario in a randomly chosen location until the expiration of the pause time then the mobile node randomly chooses another location and the speed needed to reach the new location. The speed is uniformly distributed between [0, MAXSPEED]. The node travels to the new location with the chosen speed. When the mobile node reaches the destination, the node takes a break of moving by activating the pause time. 


\section{CHAPTER 5 - SIMULATION DESIGN AND IMPLEMENTATION}

In this chapter, the following will be explained: the setup design and implementation in Network Simulator 2 (NS-2) for analyzing the multimedia traffic, such as video and voice streaming, HDTV and SDTV in MANETs and WMNs with different types of broadcast protocols with or without NC techniques. The simulation was performed using NS-2.

\subsection{Simulation Goals}

The main goals of the simulations preformed in this thesis include analyzing the behavior of multimedia traffic in ad hoc wireless mesh networks with different types of broadcast protocols with or without NC technique. Performance criteria evaluated include calculating latency, jitter and PDR by considering static and mobile scenarios for ad hoc wireless mesh networks.

A number of simulations carried out in this thesis fall into two main categories: one is with static scenarios where nodes are stable and not moving around base station(s) and the other with mobility scenarios where all nodes, except base station(s), are moving around. Simulations results are obtained by increasing/decreasing the number of base stations and changing of network scenarios.

This thesis considers four types of multimedia traffic: video streaming, voice streaming, HDTV and SDTV. The multimedia traffic is continually generated by the base station(s) and sent out to several nodes. Furthermore, all nodes act as intermediate nodes which forward received packets to the nodes that are out of the base station(s) range. 


\subsection{Simulation Parameters}

NS-2 parameters consist of three main parts; Global variables, MAC layer parameters and Physical layer parameters.

\subsubsection{Global Variables}

Certain variables are declared and initialized at the beginning of each simulation using NS-2 rather than hard-coding them in the code. Thus, variables happen to be simply accessible and simulation can be easily managed by changing their values. Moreover, the code is now more understandable with this approach. Table 5.1 shows the global variables in NS-2 Tcl simulation files with a brief explanation of the use of each variable.

Table 5.1: Global Variable of simulation

\begin{tabular}{|c|c|c|}
\hline Global Variable & Values & Description \\
\hline $\operatorname{val}($ chan) & Channel/WirelessChannel & Channel type \\
\hline $\operatorname{val}($ prop) & Propagation/TwoRayGround & $\begin{array}{c}\text { Radio propagation } \\
\text { model }\end{array}$ \\
\hline $\operatorname{val}($ netif) & Phy/WirelessPhy & Network interface type \\
\hline $\operatorname{val}(\operatorname{mac})$ & $\mathrm{Mac} / 80211$ & MAC type \\
\hline $\operatorname{val}(\mathrm{i} f q)$ & Queue/DropTail/Priqueue & Interface queue type \\
\hline $\operatorname{val}(11)$ & $\mathrm{LL}$ & Link layer type \\
\hline $\operatorname{val}(\mathrm{ant})$ & Antenna/OmniAntenna & Antenna model \\
\hline $\operatorname{val(ifqlen)}$ & 50 & $\begin{array}{l}\text { Maximum packets in } \\
\text { IFQ }\end{array}$ \\
\hline $\operatorname{val}(r p)$ & $\begin{array}{l}\text { RLNC or CODECAST (with } \\
\text { and without NC) }\end{array}$ & Routing protocol \\
\hline $\operatorname{val}(\mathrm{nn})$ & 10 & $\begin{array}{c}\text { Number of wireless } \\
\text { nods }\end{array}$ \\
\hline val (nn_bsnodes) & $1,2,3$ or 4 & $\begin{array}{c}\text { Number of base } \\
\text { stations }\end{array}$ \\
\hline $\operatorname{val}(\mathrm{x})$ & 720 or 1000 & $\mathrm{X}$ grid dimension \\
\hline $\operatorname{val}(\mathrm{y})$ & 720 or 1000 & $\mathrm{Y}$ grid dimension \\
\hline val (runtime) & 100 & Length of simulation \\
\hline
\end{tabular}




\subsubsection{MAC Layer Parameters}

The simulations use a 802.11 in the MAC layer and the MAC layer is configured for $24 \mathrm{Mbps}$. Table 5.2 shows the MAC layer variables in the Tcl simulation files with brief explanation of the use of each variable.

Table 5.2: MAC Layer Variable of simulation

\begin{tabular}{|c|c|c|}
\hline MAC Layer Parameter & Value & Description \\
\hline SlotTime_ & 0.000020 & $\begin{array}{c}\text { Sets slot time for back-off } \\
\text { window }\end{array}$ \\
\hline PreambleLength_ & 0.000010 & $\begin{array}{c}\text { Sets short inter-frame space } \\
\text { bets length of preamble in } \\
\text { bit }\end{array}$ \\
\hline PLCPHeaderLength_ & 144 & $\begin{array}{c}\text { Sets Physical Layer } \\
\text { Convergence Protocol } \\
\text { header length in bit }\end{array}$ \\
\hline PLCPDataRate_ & 48 & $\begin{array}{c}\text { Sets Physical Layer } \\
\text { Convergence Protocol rate } \\
\text { to 6 } \text { 10^6 Mbp }\end{array}$ \\
\hline basicRate & $6.0 e 6$ & Bandwidth rate of MAC \\
\hline dataRate & $24.0 \mathrm{e} 6$ & Data tra-nsfer rate for traffic \\
\hline RTSThreshold & $24.0 \mathrm{e} 6$ & Sets RTS Threshold \\
\hline ShortRetryLimit & 3000 & Sets long retries \\
\hline LongRetryLimit & 7 & Sets short retries \\
\hline
\end{tabular}

5.2.3 Physical Layer Parameters

The simulation has the following (shown in table 5.3 and 5.4) parameters to configure the physical layer and the antenna specifications.

Table 5.3: Physical Layer Variable of simulation

\begin{tabular}{|c|c|c|}
\hline Physical Layer Variable & Value & Description \\
\hline CSThresh_ & $3.16 \mathrm{e}-14$ & $\begin{array}{c}\text { Set Carrier sense range } \\
\text { where sensitivity }=-105 \\
\text { dbm }\end{array}$ \\
\hline RXThresh_ & $3.16 \mathrm{e}-14$ & Set Receive rang \\
\hline CPThresh_ & 10 & Capture threshold \\
\hline Pt_ & $1.838 \mathrm{e}-5$ & $\begin{array}{c}\text { Power needed to have } 240, \\
\text { of distance }\end{array}$ \\
\hline L & 1.0 & System loss factor \\
\hline freq_ & $2.4 \mathrm{e} 9$ & $\begin{array}{c}\text { Frequency of network, set } \\
\text { to 2.4 GHz }\end{array}$ \\
\hline
\end{tabular}


Table 5.4: Antenna Variable of simulation

\begin{tabular}{|c|c|c|}
\hline Antenna variable & Values & Description \\
\hline $\mathrm{Z}$ & 1.5 & Antenna height $=1.5$ meters \\
\hline $\mathrm{Gt}$ & 1.0 & Antenna gain of transmitter \\
\hline $\mathrm{Gr}$ & 1.0 & Antenna gain of receiver \\
\hline
\end{tabular}

\subsection{Network Topology}

Next stage is creating the topology which is used in the simulation for this thesis. First, an NS object was created (see Appendix - Figure A.1 for the creation code of NS object). $n s_{-}$variable is now used throughout the code to execute several functions and operations in the simulations.

Second, the simulation code creates a file to trace every exchanged packet between the different nodes. After that, the file is used to calculate overall delay, jitter and PDR of the network (see Appendix - Figure A.2 for the creation code of the trace file).

Third, the simulation code creates topology and topography (see Appendix Figure A.3 for the code). The first line creates a new topography variable called topo. The topo variable is used to keep track of the mobile-nodes movement in the given boundary. Second line specifies the boundaries of the area where the nodes are moving in. In the next line, a General Operations Director (God) variable is created to store information about the network's mobile nodes and the shortest path from one node to another. The number of mobile nodes is used to be an argument to this God variable.

In our topology, all communications between nodes and base station(s) are wireless throughout certain broadcast protocols which are used in our simulations. The 
simulation codes create number wireless nodes (see Appendix - Figure A.4 for the creation code) and specify the location of each node in the network area by calling it a mobility file which was created previously by the Bonnmotion scenario generator. Also, simulation codes create a base station(s) to broadcast multimedia traffic which is used in the simulation.

In NS-2, every mobile node needs to be configured before it is created. These options are configured once. The "applicable to all mobile nodes" in the Tcl script is created. These options are configured in the following way in NS-2 simulation (see Appendix - Figure A.5).

Next step is to create a multimedia traffic (see Appendix - Figure A.6 which shows an example of generating an audio streaming traffic for the RLNC protocol by the base station(s) to be broadcasted to all nodes and how all the nodes are assigned to receive audio streaming traffic from the base station(s)).

In order to create a base station, first of all a User Datagram Protocol (UDP) agent is created and attached to all wireless node. Then, a number of multimedia wireless nodes are set to receive broadcasted encoded packets from the base station(s). In this code, number of nodes is set to "10". For receiving traffic, a NULL agent is created and then attached to the base station. In order to send traffic from the base station to wireless nodes, the UDP agent of the base station must be connected to a respective NULL agent of wireless node in the RLNC code. The UDP agent of base station is connected to the NULL agent of wireless node. Similarly, UDP agent of base station must be connected to all other wireless nodes NULL agents. However, in PDP and SMF the base station broadcasts their packets using a special broadcast address and port i.e. "699" as shown in 
Appendix - Figure A.7. Also, each UDP agent is attached to CBR agent which specifies the packet size and the data rate as shown in Table 5.5.

Table 5.5: Traffic Specifications

\begin{tabular}{|c|c|c|c|c|}
\hline Application & Data Rate (Mbps) & Protocol & MSDU size (Bytes) & Max Delay (ms) \\
\hline SDTV $^{\mathbf{a}}$ & $4-5$ & UDP & 1500 & 200 \\
\hline HDTV $^{\mathbf{a}}$ & $19.2-24$ & UDP & 1500 & 200 \\
\hline $\begin{array}{c}\text { Video } \\
\text { Streaming }\end{array}$ & $0.1-4$ & UDP & 512 & 200 \\
\hline $\begin{array}{c}\text { Audio } \\
\text { Streaming }\end{array}$ & $0.064-0.256$ & UDP & 208 & 200 \\
\hline
\end{tabular}

a. values specify HDTV, SDTV and video streaming [46]

b. values specify a MPEG audio streaming [45]

Last, an important step includes scheduling events for base station(s) and wireless nodes. It consists of two steps;

1. "start" time for traffic agent.

2. "stop" time of traffic agent.

See Appendix - Figure A.8 for the code.

\subsection{Protocols' Specifications}

The final stage is to specify the protocols which were used in the simulations done for the current thesis. The seven protocols that were used in the simulations were as following; PDP, PDP/XOR, PDP/RS, SMF, SMF/XOR, SMF/RS and RLNC.

The table (Table 5.6) below shows parameters and variables that should be specified in order to have PDP and SMF with and without NC. 
See Appendix - Figure A.9 for a code example for PDP and Appendix - Figure A.10 and Figure A.11 for codes for the RLNC specification to be used in our thesis.

Table 5.6: PDP and SMF Specifications

\begin{tabular}{|c|c|c|c|c|c|c|}
\hline Variables & \multicolumn{7}{|c|}{ Value } \\
\hline hello_int_ & 1 & 1 & 1 & 1 & 1 & 1 \\
\hline PDP_ & true & True & true & false & false & false \\
\hline coding_ & false & True & true & false & true & true \\
\hline RS_coding_ & false & False & true & false & false & true \\
\hline Protocol & PDP & PDP/XOR & PDP/RS & SMF & SMF/XOR & SMF/RS \\
\hline
\end{tabular}




\section{CHAPTER 6 - SIMULATIONS AND EVALUATION}

The chapter explains the simulation setups employed to study the effect with and without NC techniques on the multimedia traffic of ad hoc wireless mesh networks. Different simulations were carried out with varying multimedia applications and number of mobile nodes. Results were obtained from ad hoc wireless mesh networks having different multimedia applications with seven broadcast protocols:

1. SMF Protocol without NC

2. SMF with XOR-NC Protocol

3. SMF with RS-NC Protocol

4. PDP Protocol without NC

5. PDP with XOR-NC Protocol

6. PDP with RS-NC Protocol

7. RLNC Protocol

\subsection{General Simulation Setup}

The Base Station(s) (BS) is/are placed in a $720 \mathrm{~m} \times 720 \mathrm{~m}$ or $1000 \mathrm{~m} \times 1000 \mathrm{~m}$ area where the BS(s) with antenna range of $230 \mathrm{~m}$ is/are geographically centered and evenly distributed in the simulation area. The location of BS(s) is shown in Table 6.1 and Figure 6.1. The simulations are divided into two parts: mobility scenario and static scenario. The static scenario is called "scenario\#1" where the mobile wireless nodes remain in the same locations around 1-4 BS(s). The wireless nodes randomly surround the BS(s); where ten wireless nodes surround the $\mathrm{BS}(\mathrm{s})$ in $720 \mathrm{~m} \times 720 \mathrm{~m}$ area or hundred wireless nodes surround the BS(s) in $1000 \mathrm{~m} \times 1000 \mathrm{~m}$ area. The mobility scenario is called "scenario $\# 2$ " 
where 2 BSs are randomly surrounded by ten wireless nodes in $720 \mathrm{~m} \times 720 \mathrm{~m}$ area or hundred wireless nodes $1000 \mathrm{~m} \times 1000 \mathrm{~m}$ area that move individually around the whole simulation area based on the Random Waypoint mobility model, with speed selected between $1-10 \mathrm{~m} / \mathrm{s}$ and 0 pause time. Multimedia traffic is generated and sent by the BS(s) to the mobile nodes where mobile nodes are receivers in both static and mobile scenarios. Moreover, mobile nodes may act as relays and broadcast traffic to other nodes which are out-of-range of the $\mathrm{BS}(\mathrm{s})$. Also, a BS may act as a relay when it receives a new traffic from another BS.

Table 6.1: System Parameters

\begin{tabular}{|c|c|c|c|c|}
\hline \multirow{3}{*}{$\begin{array}{l}\text { Parameters } \\
\text { Number of BS } \\
\end{array}$} & \multicolumn{4}{|c|}{ Routing } \\
\hline & $S M F$ & $P D P$ & $M A C$ & rotocol \\
\hline & 1 & 2 & 3 & 4 \\
\hline Location of BSs $(\mathrm{x}, \mathrm{y})$ & $1:(360,360)$ & $\begin{array}{l}1:(180,360) \\
2:(540,360)\end{array}$ & $\begin{array}{l}1:(180,180) \\
2:(360,540) \\
3:(540,180)\end{array}$ & $\begin{array}{l}1:(180,180) \\
2:(180,540) \\
3:(540,180) \\
4:(540,540)\end{array}$ \\
\hline
\end{tabular}

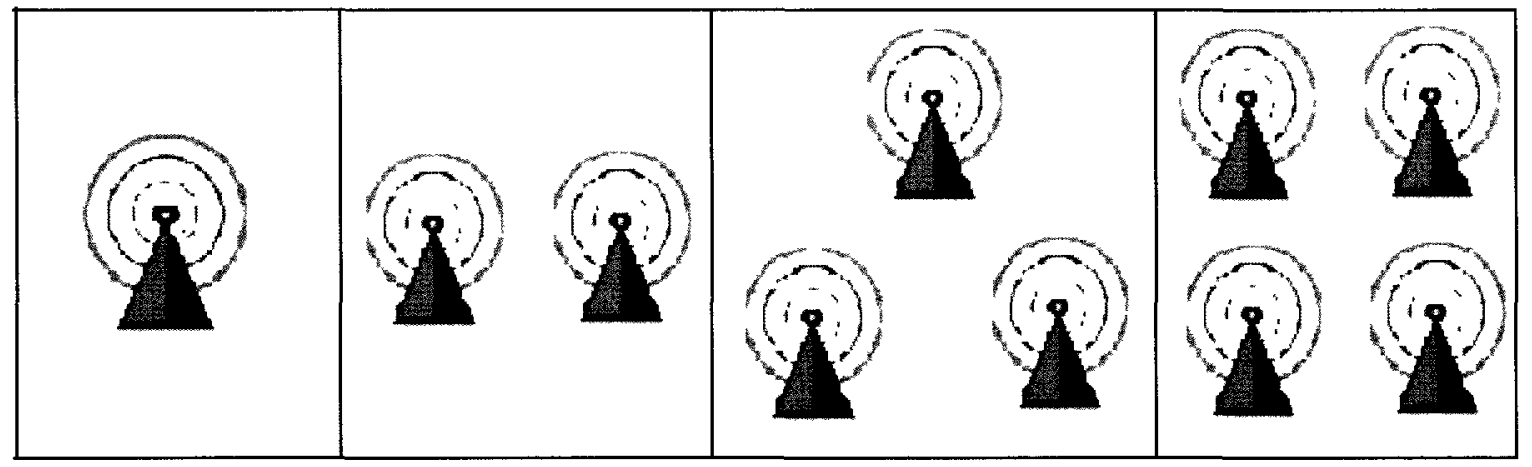

Figure 6.1: Base Station Arrangements 


\subsection{Performance Parameters}

The following performance parameters for each scenario for all simulation were calculated in order to differentiate the performance difference for the multimedia traffic.

- Packet Delivery Ratio

- Average Delay/ Latency

- Jitter

Where all performance results are averaged over ten different runs and each simulation time is 100 seconds.

\section{Packet Delivery Ratio (PDR)}

PDR for broadcast protocols is defined as the ratio of the total number of packets received to the number of packets meant to be received. This can be calculated by counting the total number of packets received by all nodes and dividing by the number of packets sent by each source multiplied by the number of receivers, as shown in below equation where $N$ is the number of nodes in the network and " $i$ " is the number of received packets.

$$
P D R=\frac{\sum_{i=1}^{N} \operatorname{Re} \text { ceivedPackets }{ }_{\iota}}{\text { NumberOfSourcePackets } \times \text { NumberOf } \operatorname{Re} \text { ceivers }}
$$

In case of $\mathrm{NC}$, packets are considered to be received packet when the receiving node successfully decodes the received packets and recover the desired information.

\section{Average End-to-End Delay/Latency}

The average latency of a packet is defined as the average amount of time elapsed between the source sending a packet and all receivers decoding the packet and is shown 
in the equation below where $N$ is the number of receiving nodes for the broadcasted packet:

$$
\text { Latency }=\frac{\sum_{i=1}^{N} \text { TimeDecoded }_{\imath}}{N}-\text { TimeSent }
$$

The average latency for all the packets in the network can then be described as the sum of all latencies for each packet divided by the number of sent packets shown in the equation below where $J$ is the number of sent packets.

$$
\text { AveLatency }=\frac{\sum_{i=1}^{J} \text { Latency }_{i}}{J}
$$

At the source, different factors add latency, such as the procedures of encoding packets using one of the $\mathrm{NC}$ techniques. On the wireless network level, packet is queued in different routers, forwarded to different nodes; all these factors increase the latency. On the receiving nodes, corresponding packets are needed to be decoded which also adds to end-to-end delay.

Jitter

Jitter is known as the variance in the latency between packets from the same data flow. Jitter can negatively impact the real time applications, such as audio and video streaming. Jitter can be created through different reasons; variance in Serialization Delays which is caused by the variance in the packet sizes, variance in per-packet Queue Delay which is caused by packet spacing from multiple sources at a single interface (destination), or packets taking different routes from source to destination because of routing issues. A smaller jitter shows a higher quality flow. 
Jitter is calculated by the below equation of variance:

$$
\text { Jitter }=\frac{1}{J-1} \sum_{i=1}^{J}\left(X_{\imath}-X\right)^{2}
$$

Where $J$ is the number of received packets, $X_{t}$ is the received packet latency and $X$ is the average latency.

\subsection{Simulations Results and Evaluation}

This part of thesis report includes results from simulations carried out and their evaluation. Simulations topology and network traffic has been explained in earlier sections. The simulations were carried out with varying applications, number of BSs and speed of the mobile nodes. Each simulation result is the average of ten simulation runs with different node locations which are chosen randomly by Bonnmotion [47]. In addition, $95 \%$ confidence intervals are used for the simulation results.

For evaluating multimedia broadcast applications' quality, two performances associated main parameters need to be kept in mind, which are as follow:

- Latency tolerance for the multimedia clients was practically established to be as shown in table 5.5 in Chapter 5.

- Multimedia clients are reasonably happy with having the highest PDR which provides higher voice and video quality.

\subsubsection{0-Wireless-Node Scenarios}

First set of simulations were established with ten wireless nodes (Multimedia Clients) placed around BS(s) in order to evaluate the performance difference of several multimedia broadcast applications measured at each wireless node over the ad hoc 
wireless mesh network. The first set of simulations is divided into two sections; static scenarios and mobility scenarios.

\subsubsection{Static Scenarios}

The following figures contain results obtained from different simulations with different multimedia broadcast applications (Video Streaming, Voice Streaming, SDTV and HDTV traffic) and with varying number of BSs. Since each simulation repeated ten times with different location of the wireless nodes, as a result average of PDR, latency and jitter are shown in the following figures with their confidence intervals.

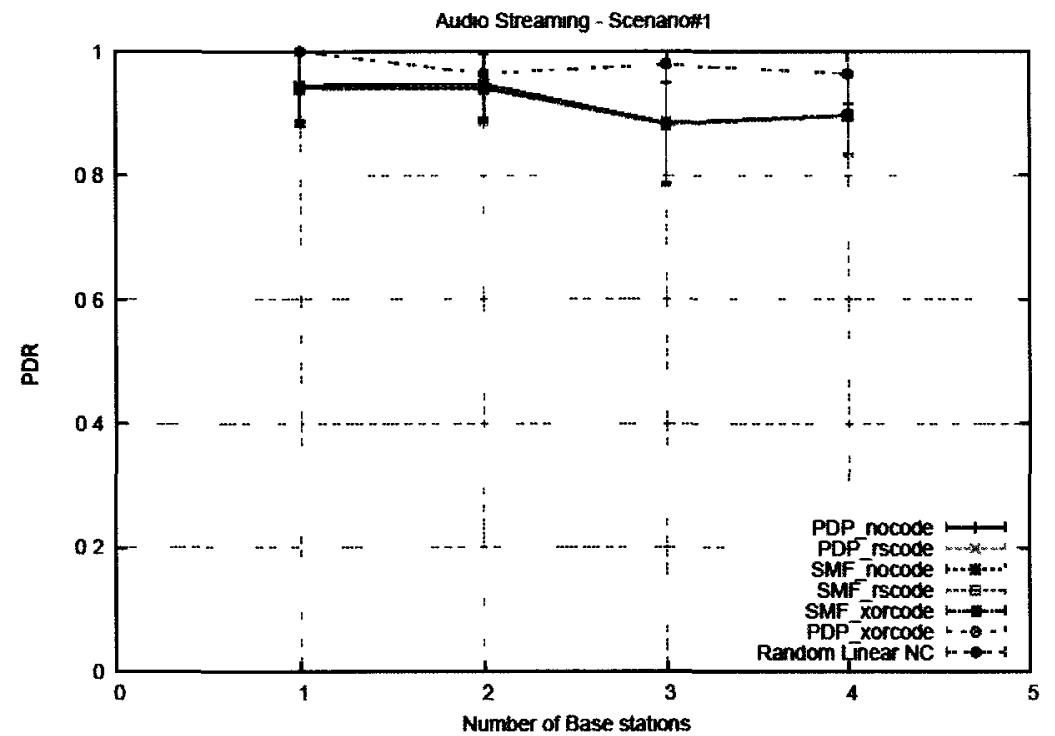

Figure 6.2: PDR for Audio Streaming (Static Scenario - Low Density) 


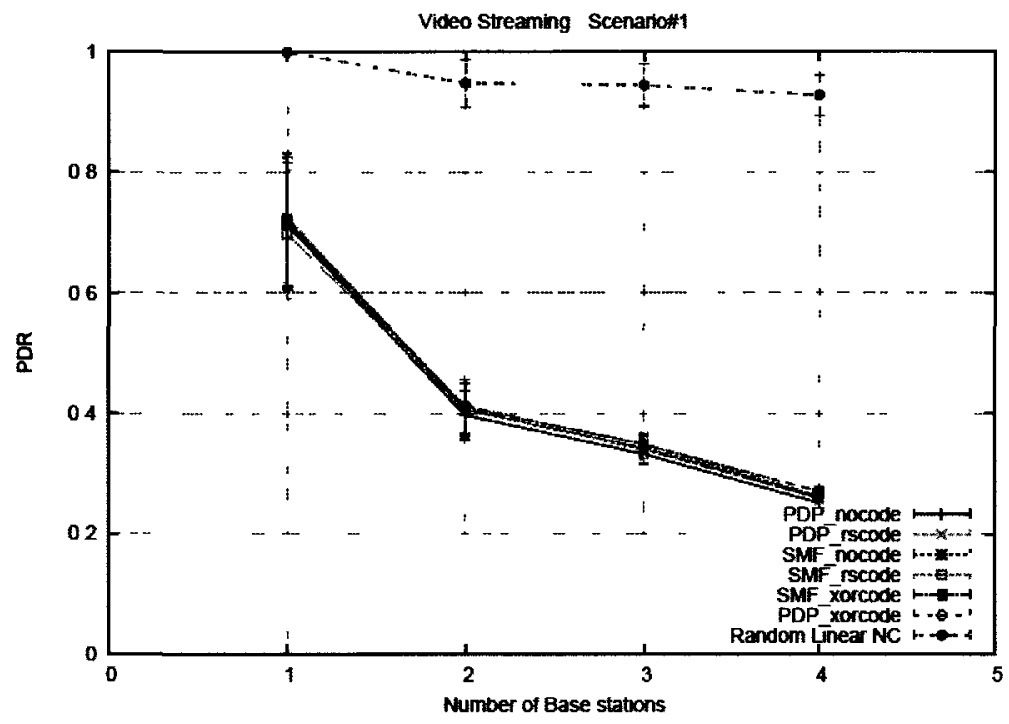

Figure 6.3: PDR for Video Streaming (Static Scenario - Low Density)

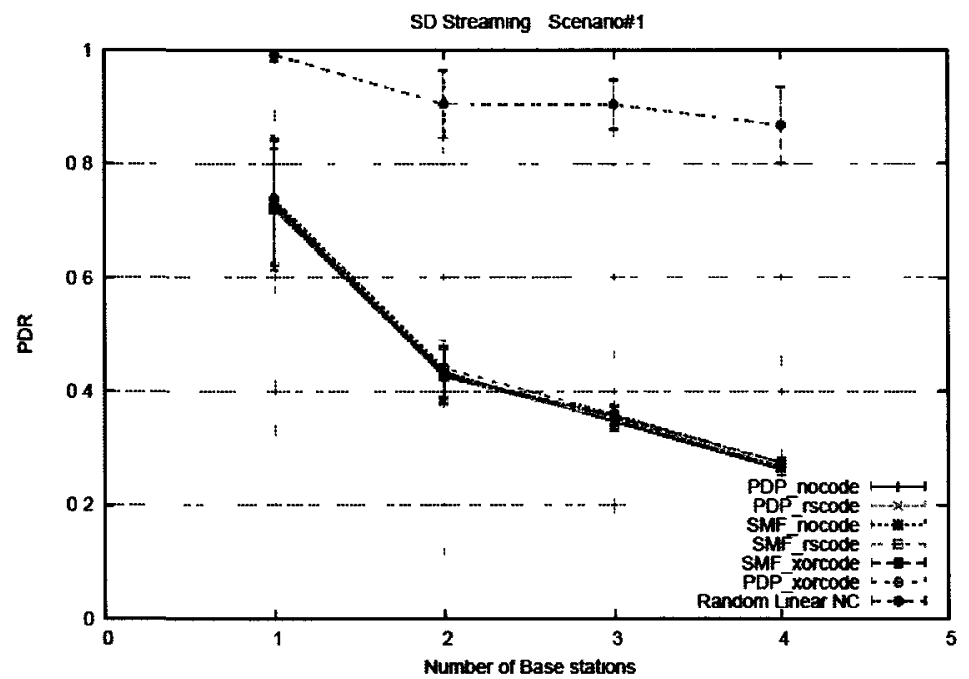

Figure 6.4: PDR for SDTV (Static Scenario - Low Density) 


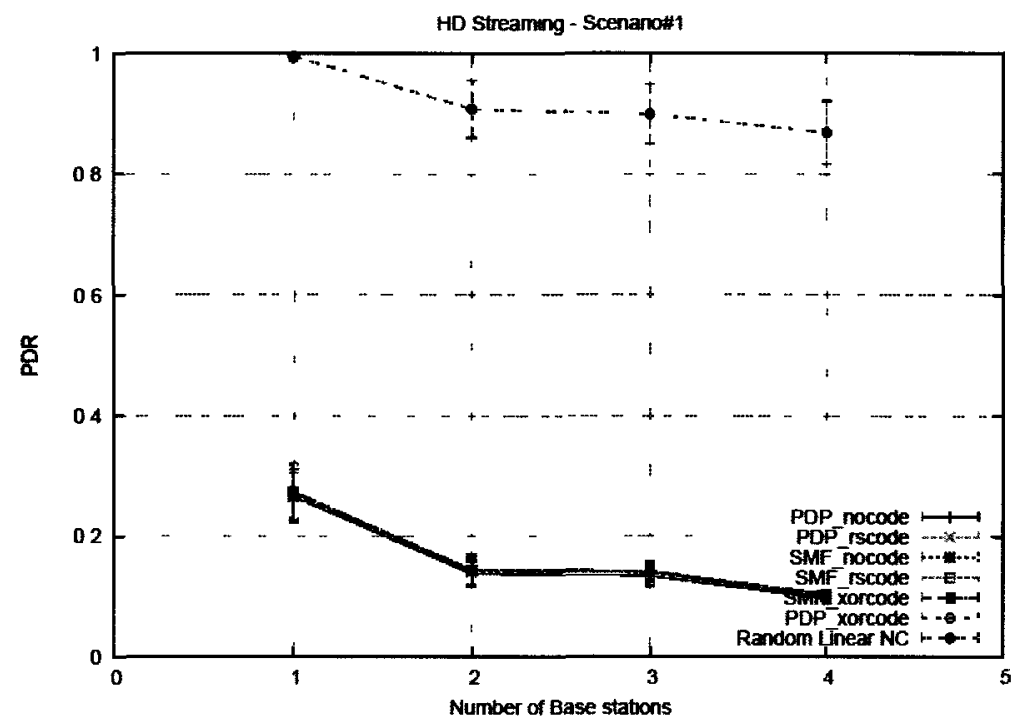

Figure 6.5: PDR for HDTV (Static Scenario - Low Density)

Figure 6.2, Figure 6.3, Figure 6.4 and Figure 6.5 show the PDR results for Scenario\#1 for several multimedia traffic; audio streaming, video streaming, SDTV and HDTV, respectively. From the results, it is clear that PDR for RLNC protocol is the highest for the tested multimedia broadcast applications. For example, in audio streaming applications the PDR of RLNC protocol decreases approximately from $100 \%$ to $96 \%$ as number of BSs (senders) increases, but still remains the highest compared to the PDR of the other protocols. The reason of this result is that SMF and PDP protocols without NC techniques flood the network with packets. As a result, increasing the number of BSs will increase delivering un-necessary packets which decrease the PDR of the whole network. Moreover, by increasing the number of BSs, the PDR of SMF and PDP protocols with $\mathrm{NC}$ techniques is negatively impacted as the receiver needs more information in order to decode the received encoded packets, where the needed information are not always 
available. On the other hand, the RLNC protocol is not mainly affected (slight deccrease) because the RLNC protocol depends on the coefficients (which are added in the headers of the encoded packets during the encoding procedure to decode the encoded packets) rather than the overhearing opportunity technique which is used in XOR and RS network coding techniques. So, the decoding procedure will not mainly be affected by the number of BSs.

Similarly, the PDR results of RLNC for other applications (e.g., video streaming, HDTV and SDTV) remain the highest. On the other hand, the PDR results of the rest of the protocols are overlapping and decrease by increasing number of BSs. Figure 6.3 shows that the PDR ranges for the tested protocols (excluding RLNC) are approximately between $26 \%$ and $71 \%$ for video streaming applications, as by increasing the number of BSs the PDR result decreases. Similarly, Figure 6.4 and Figure 6.5 show that the PDR ranges for the tested protocols (excluding RLNC) are approximate between $27 \%$ and $73 \%$ for SDTV applications, and between $10 \%$ and $26 \%$ for HDTV applications. The results show that by increasing the size of data packets and the data rates, the PDR of the RLNC remains the highest and stays above $80 \%$, where the PDR results of other tested protocols drop below $80 \%$ in video streaming and SDTV applications and below $30 \%$ in HDTV applications.

Figure 6.6, Figure 6.7, Figure 6.8 and Figure 6.9 show the latency results for Scenario\#1 for several multimedia broadcast applications. For example, in the audio streaming applications the latency values slightly increase for all tested protocols as number of BSs increases. However, the latency result of RLNC remains the lowest compared to all other values and is acceptable to audio streaming applications. The 
latency results of SMF and PDP without NC increase and remain as an acceptable value $(<200 \mathrm{~ms})$ till number of BSs more than 2 BSs. The reason of this result is that number of flooded packets in the network increases by increasing number of BSs, which increases the queue size at the receiver size (increases the waiting time for a packet to be delivered to the application layer). As a result of this, the latency increases.

Moreover, the latency results of SMF and PDP protocols with NC techniques is way higher and not acceptable ( $>200 \mathrm{~ms})$ for audio streaming applications due to the large size of the queue at the receiver when number of BSs increases and the needed time to encode and decode packets.

Similarly, the latency results of RLNC for the other applications (e.g., video streaming, HDTV and SDTV) remain the lowest. On the other hand, the latency results of the other tested protocols decreases as number of BSs increases and becomes more acceptable for multimedia applications. The reason of this is that the by using these multimedia broadcast applications which have higher data rate, the packets will be delivered faster than in case of audio streaming applications. Moreover, in case of SMF and PDP protocols with $\mathrm{NC}$ techniques the time of a receiver to receive the needed information in order to decode the received encoded packet is shorter compared to audio streaming applications. Furthermore, the latency of HDTV applications using RLNC appears to be the lowest among video streaming and SDTV applications. The reason of this result is that HDTV applications have a high data rate which allows packets to be delivered faster than any other applications to all other nodes. Also, the latency values of PDP protocols whether using NC technique or not is lower than SMF protocols with/without NC in HDTV applications. The reason of this is that the node needs to 
choose which neighbors should rebroadcast a data packet taking into account which neighbors have already received in PDP protocol. In SMF, the node wastes more time in indicating a set of nodes to deal with by sending a HELLO messages to its neighbors which also increases the size of the HELLO message.

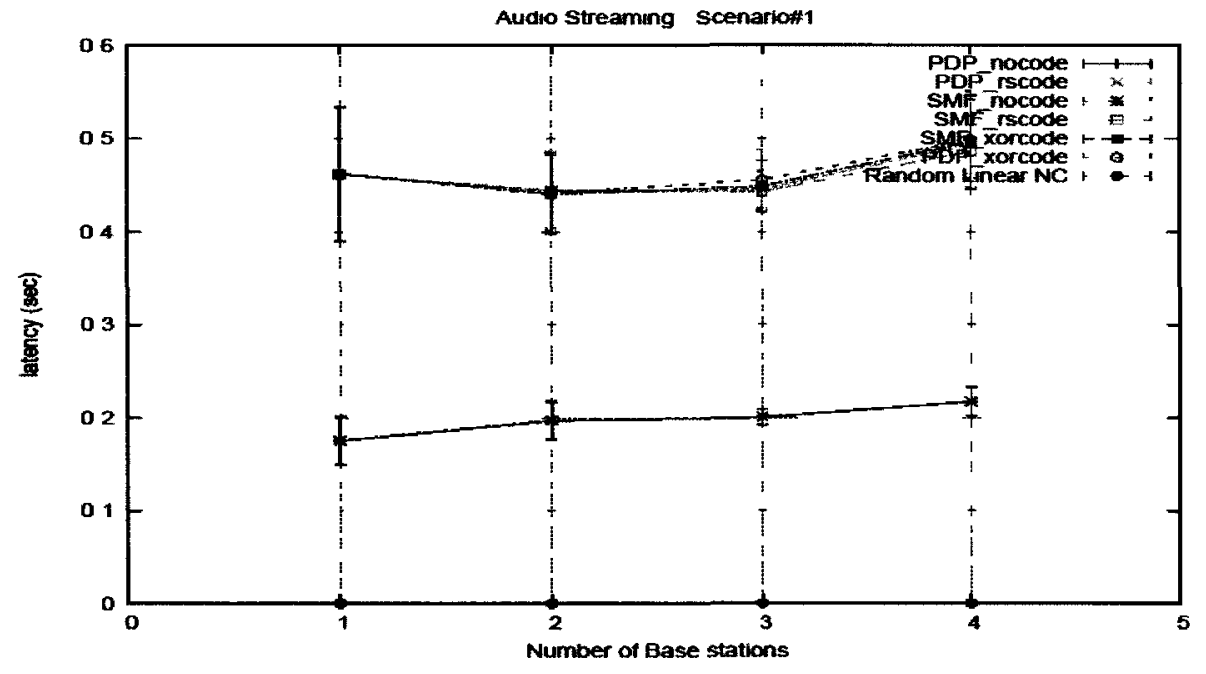

Figure 6.6: Latency for Audio Streaming (Static Scenario - Low Density) 


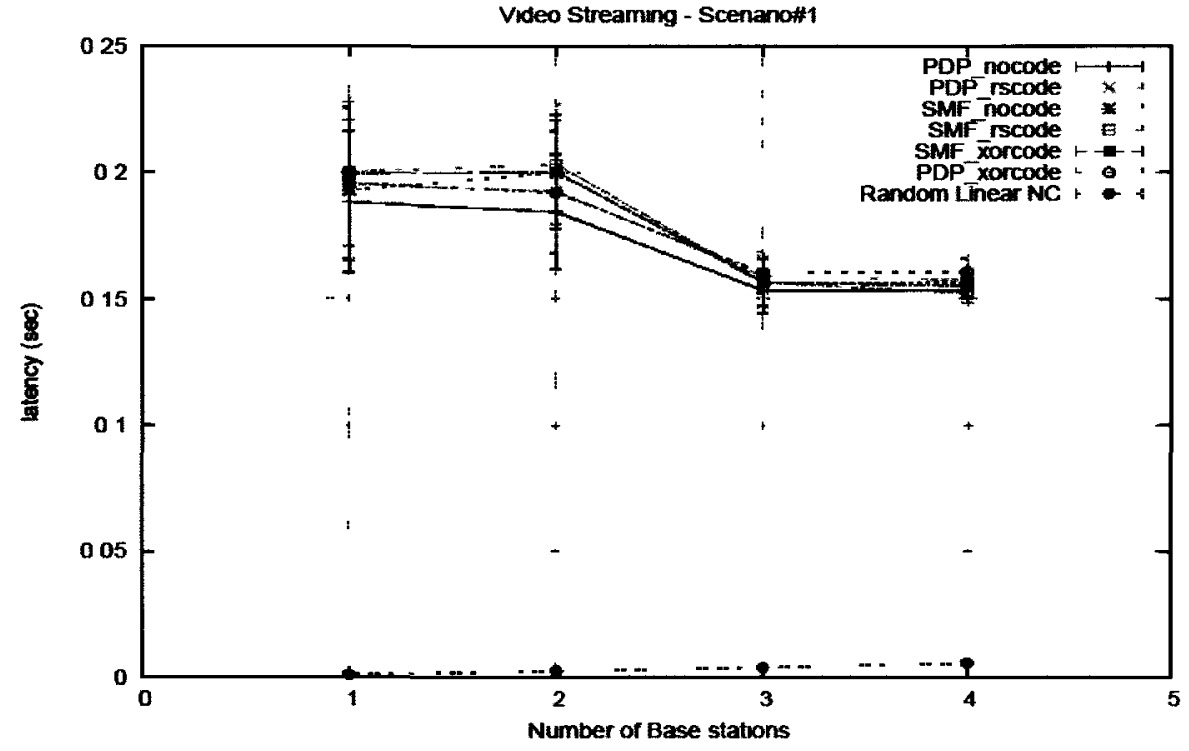

Figure 6.7: Latency for Video Streaming (Static Scenario - Low Density)

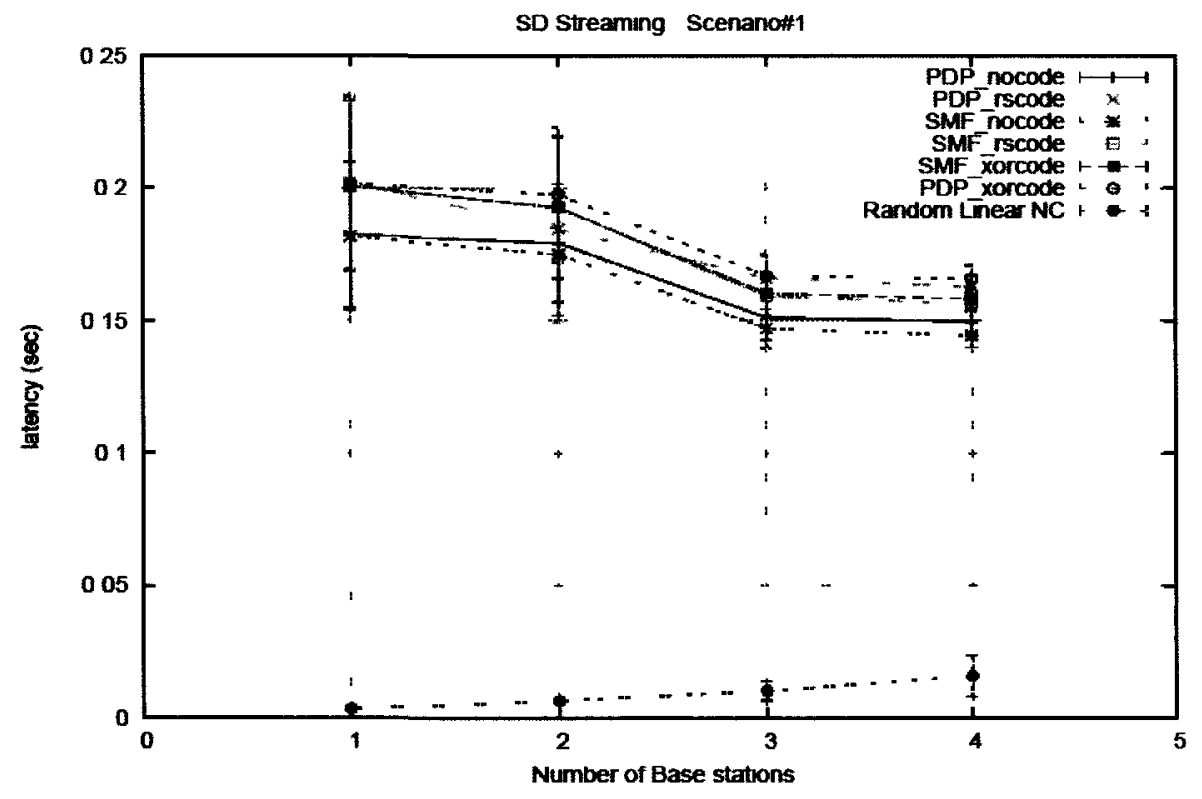

Figure 6.8: Latency for SDTV (Static Scenario - Low Density) 


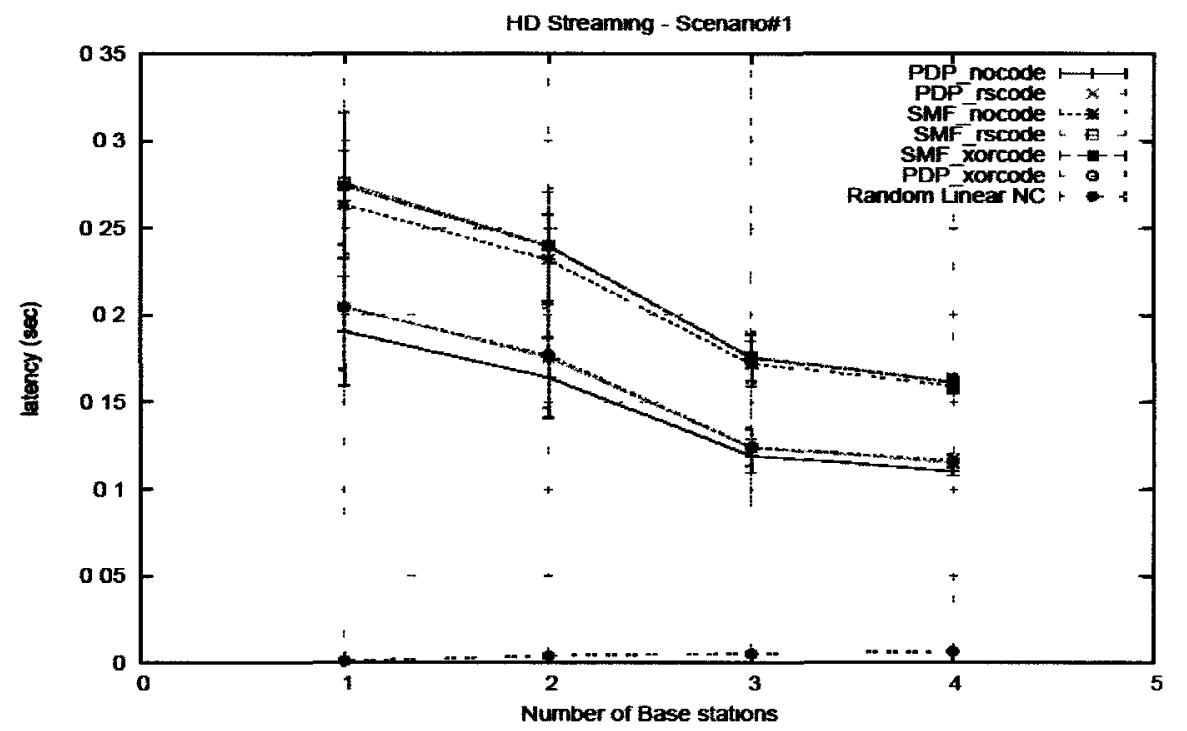

Figure 6.9: Latency for HDTV (Static Scenario - Low Density)

Figure 6.10, Figure 6.12, Figure 6.13 and Figure 6.14 show the jitter results for scenario\#1 for different multimedia broadcast applications. From the results, it is clear that the jitter values of SMF and PDP with NC are the highest compared to the other jitter values. The reason of this result is due to the dependency on the opportunistic listening mechanism which impacts the differences of the latency of the received packets, where all receiving nodes wait for overheard packets to successfully decode the received encoded packets. On the other hand, RLNC has the lowest jitter in all multimedia broadcast applications as the receiving nodes use the coefficients which are stored in the header to decode the received encoded packets. As a result, this will decrease the jitter of the network compared to the jitter values of all other tested protocols. Moreover, the jitter results of SMF and PDP without NC have also a low value but higher than RLNC. The reason of this result is that some packets may be queued in different intermediate nodes, 
or forwarded to different nodes; these factors have an impact on the latency which impacts the jitter.

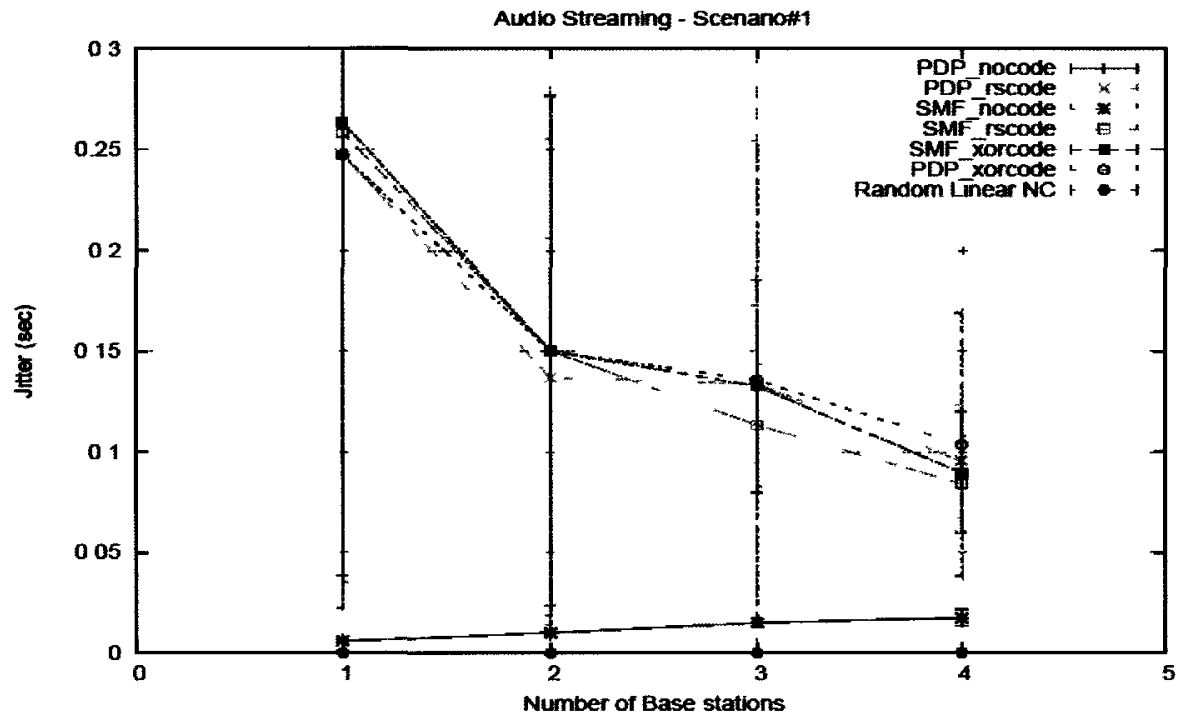

Figure 6.10: Jitter for Audio Streaming (Static Scenario - Low Density)

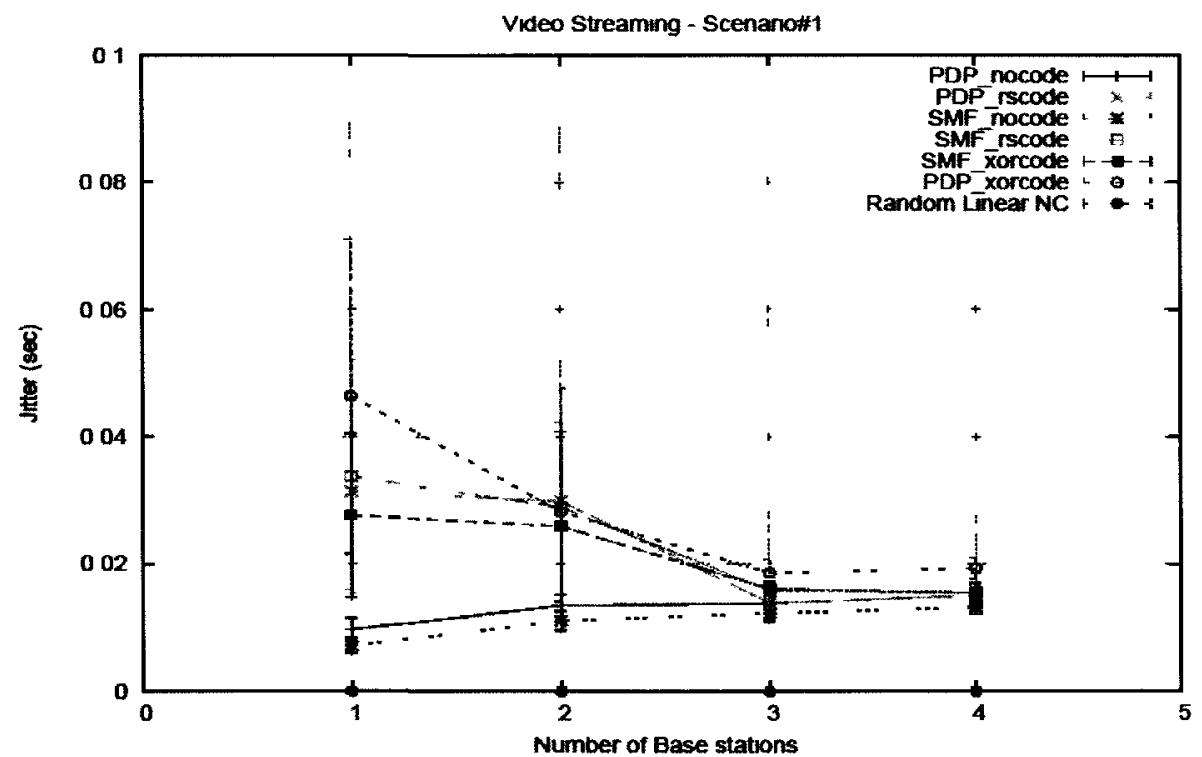

Figure 6.11: Jitter for Video Streaming (Static Scenario - Low Density) 


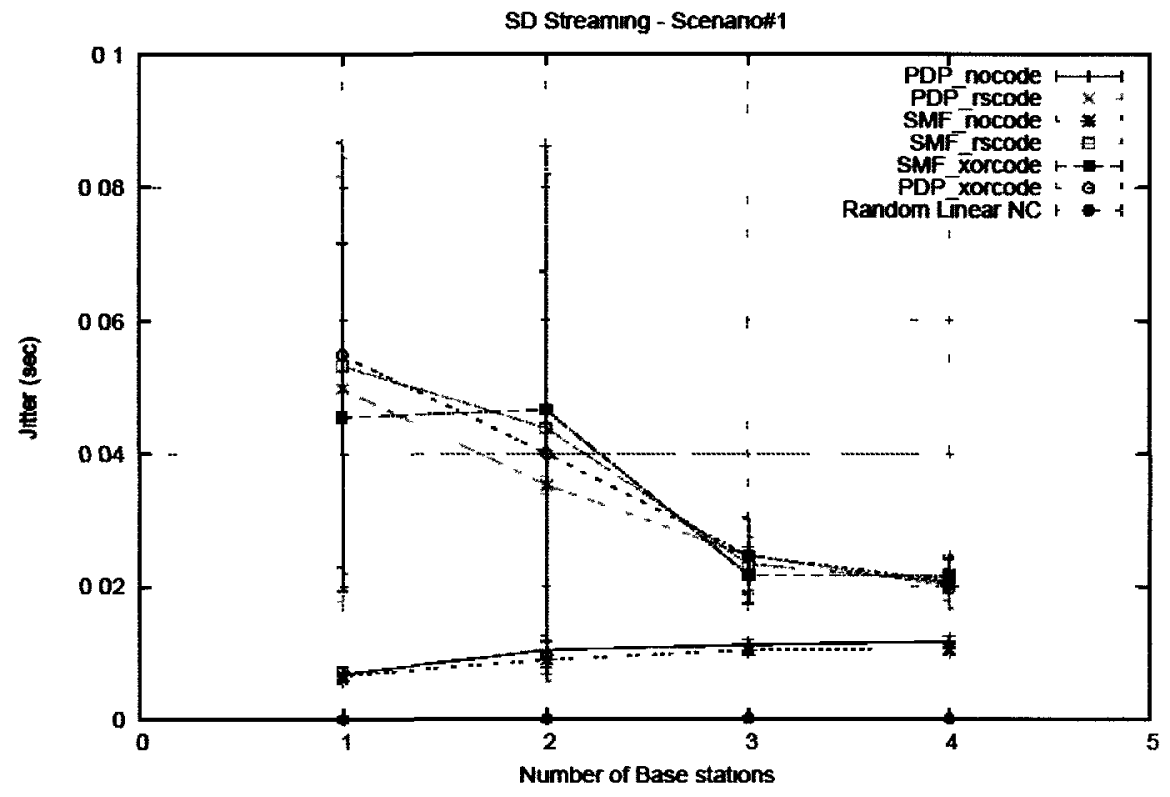

Figure 6.12: Jitter for SDTV (Static Scenario - Low Density)

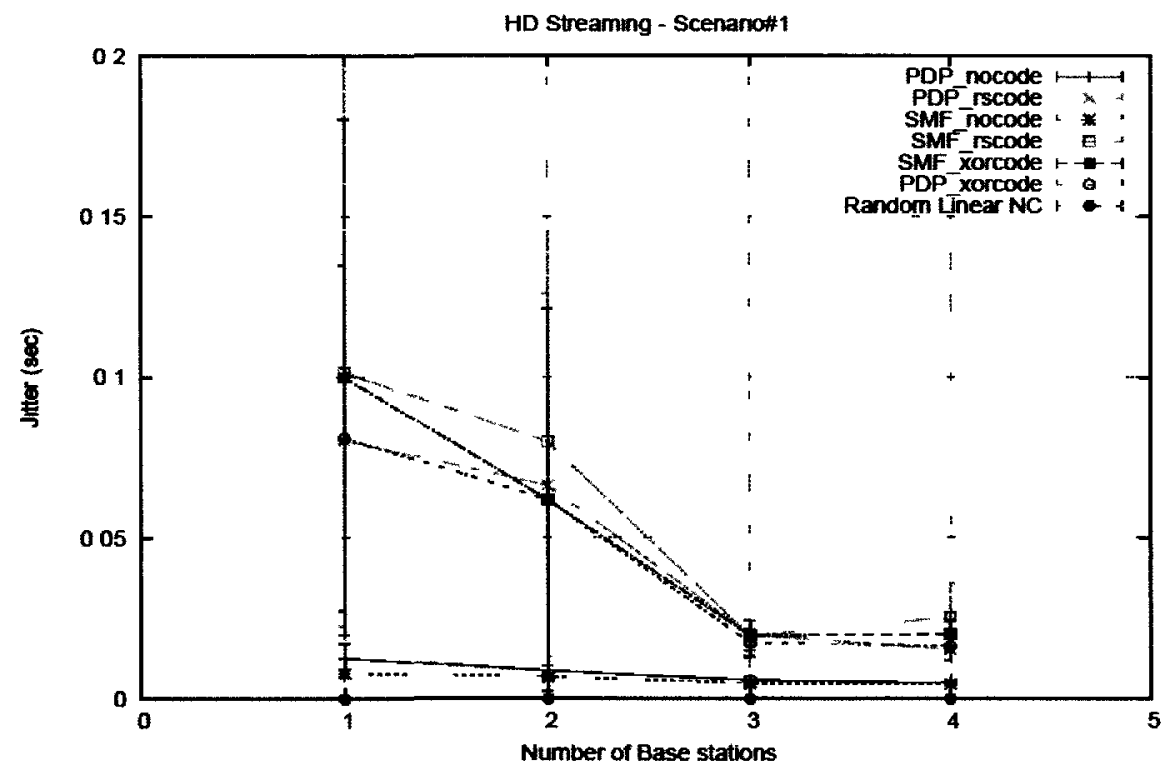

Figure 6.13: Jitter for HDTV (Static Scenario - Low Density) 
Based on the previous results, we conclude that the best protocol to be used for multimedia broadcast applications in this scenario is the RLNC. The reason of this is that RLNC protocol has the highest value of PDR and the lowest values of latency and jitter. As a result, the RLNC protocol improves the overall performance of the multimedia broadcast applications. Based on the previous results, we conclude that the best protocol to be used for multimedia broadcast applications in this scenario is the RLNC. The reason of this is that RLNC protocol has the highest value of PDR and the lowest values of latency and jitter. As a result, the RLNC protocol improves the overall performance of the multimedia broadcast applications. Moreover, the results showed that the PDP and SMF with/without NC perform badly with multimedia broadcast applications that have high data rates and large packet sizes. As a result, the rest of the results for video streaming, only SDTV and HDTV using RLNC are measured.

\subsubsection{Mobility Scenarios}

The following figures contain results obtained from different simulations with different multimedia applications with varying number of BSs. Since each simulation repeated ten times with different locations of the wireless nodes, as a result average of PDR, latency and jitter are shown in the following figures with their confidence intervals. The results for video streaming, SDTV, and HDTV applications will be only tested with RLNC as we proved that the best protocol to be used for multimedia broadcast applications is the RLNC in section 6.3.1.1 


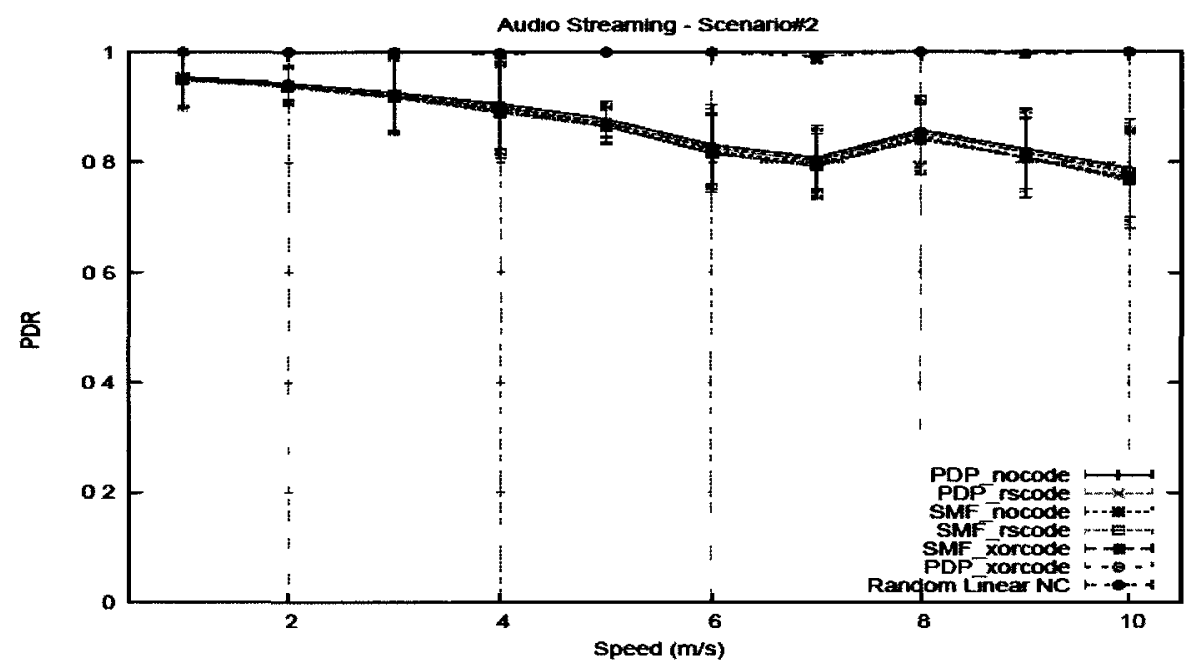

Figure 6.14: PDR for Audio Streaming (Mobile Scenario - Low Density)

Figure 6.14, Figure 6.15, Figure 6.16 and Figure 6.17 show the PDR results for Scenario\#2. From Figure 6.14, it is clear that PDR for RLNC protocol is the highest for audio streaming applications. The PDR of RLNC protocol approximately reaches $100 \%$ over various speeds. On the other hand, the PDR results of the rest of the tested protocols are overlapping and decrease by increasing the speed of mobile wireless nodes $(\approx 80 \%$ for $10 \mathrm{~m} / \mathrm{s}$ ). The reason is that SMF and PDP protocols without $\mathrm{NC}$ techniques depend on neighbors' information. As a result, increasing the speed of the wireless nodes will negatively affect the delivery of the packets as the wireless mobile nodes keep moving around in the simulation area. Similarly, SMF and PDP protocols with NC techniques depend on the opportunistic listening mechanism (to collect information to decode the encoded packets) which is also affected by the speed of the mobile wireless nodes. By increasing the speed, the opportunity of overhearing useful packets will decrease. Moreover, wireless mobile node (intermediate node) could move away from its neighbor 
area while it performs the encoding procedure which depends on opportunistic coding mechanism. As a result, the intermediate node could broadcast an encoded packet to its new neighbor area, and none of the neighbor could successfully decode this packet. Based on previous reasons, the decoding opportunities will decrease. This leads to a decline of the PDR. However, the RLNC protocol depends on the coefficients rather than the overhearing opportunity which are added in the headers of the encoded packets during the encoding procedure to decode the encoded packets. So, the decoding procedure will not be affected by the speed of the mobile wireless nodes.

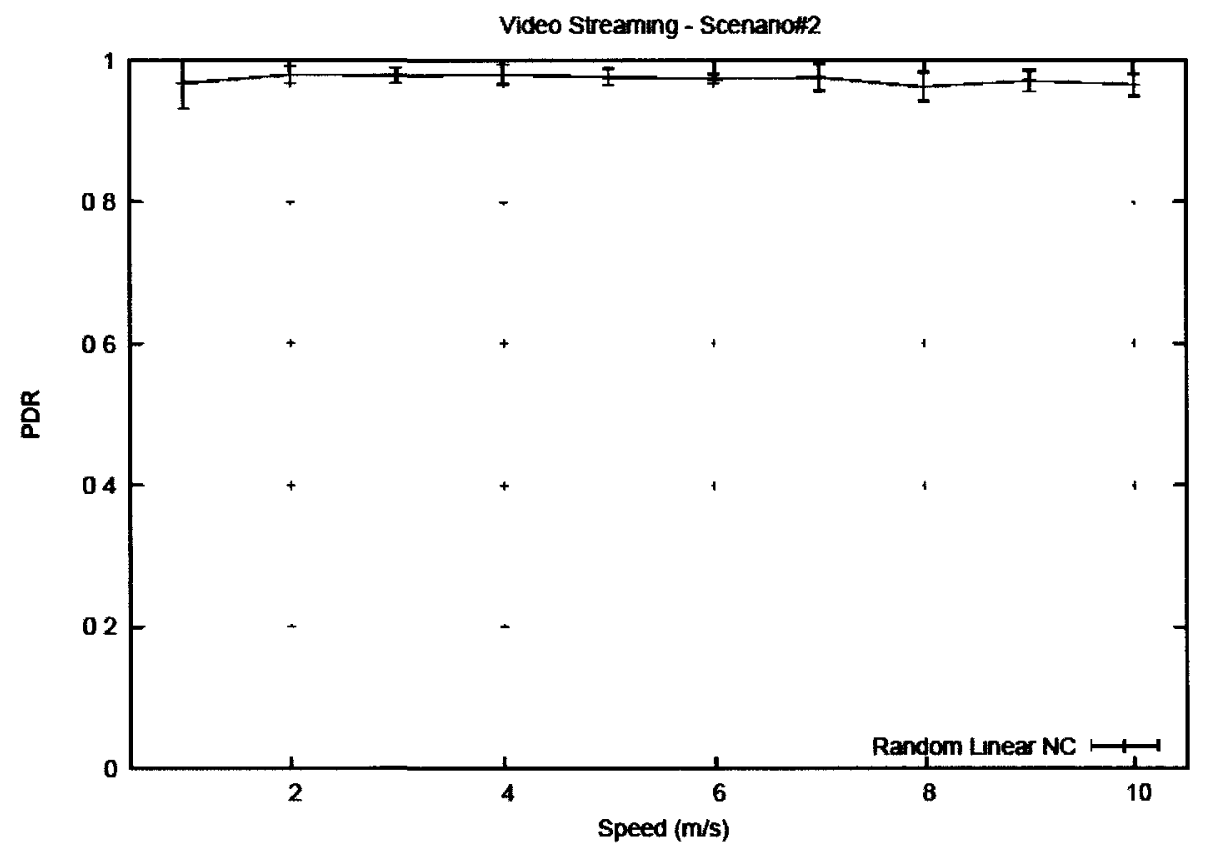

Figure 6.15: PDR for Video Streaming (Mobile Scenario - Low Density) 


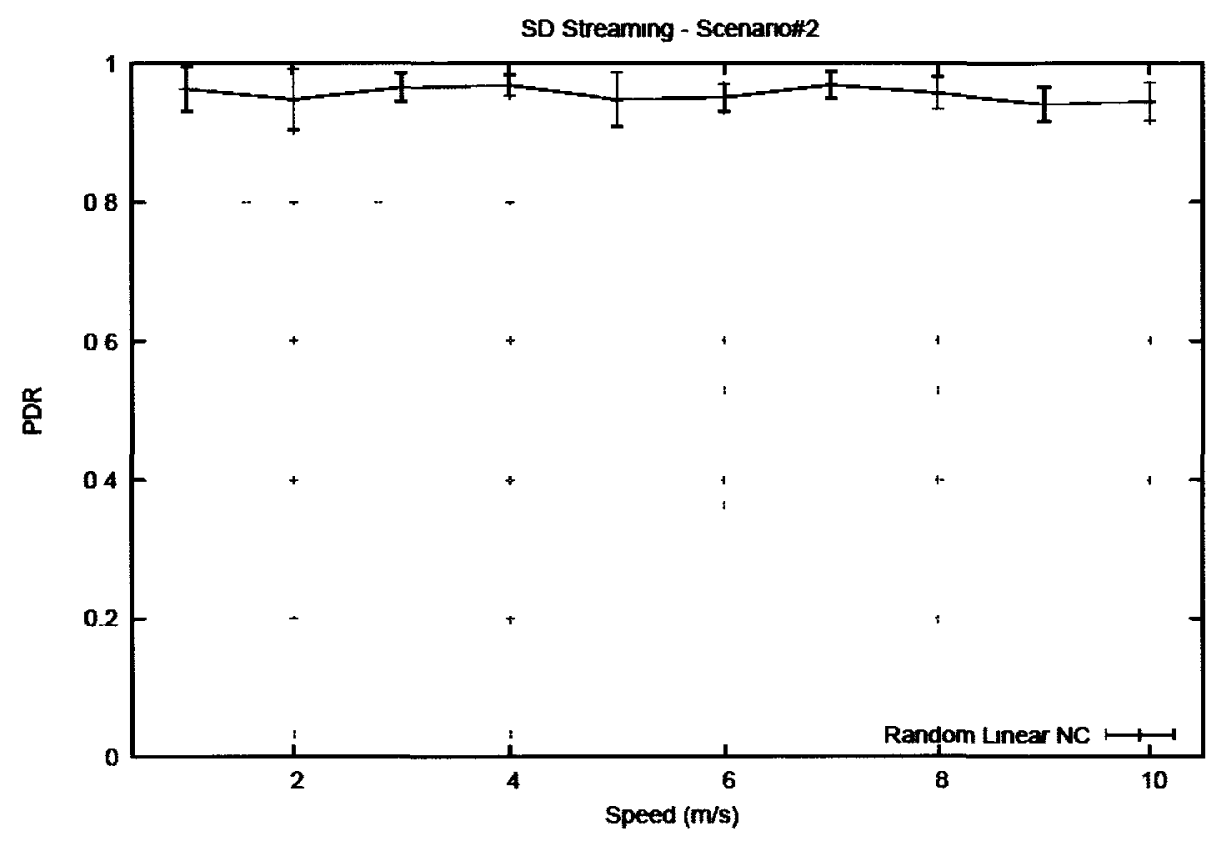

Figure 6.16: PDR for SDTV (Mobile Scenario - Low Density)

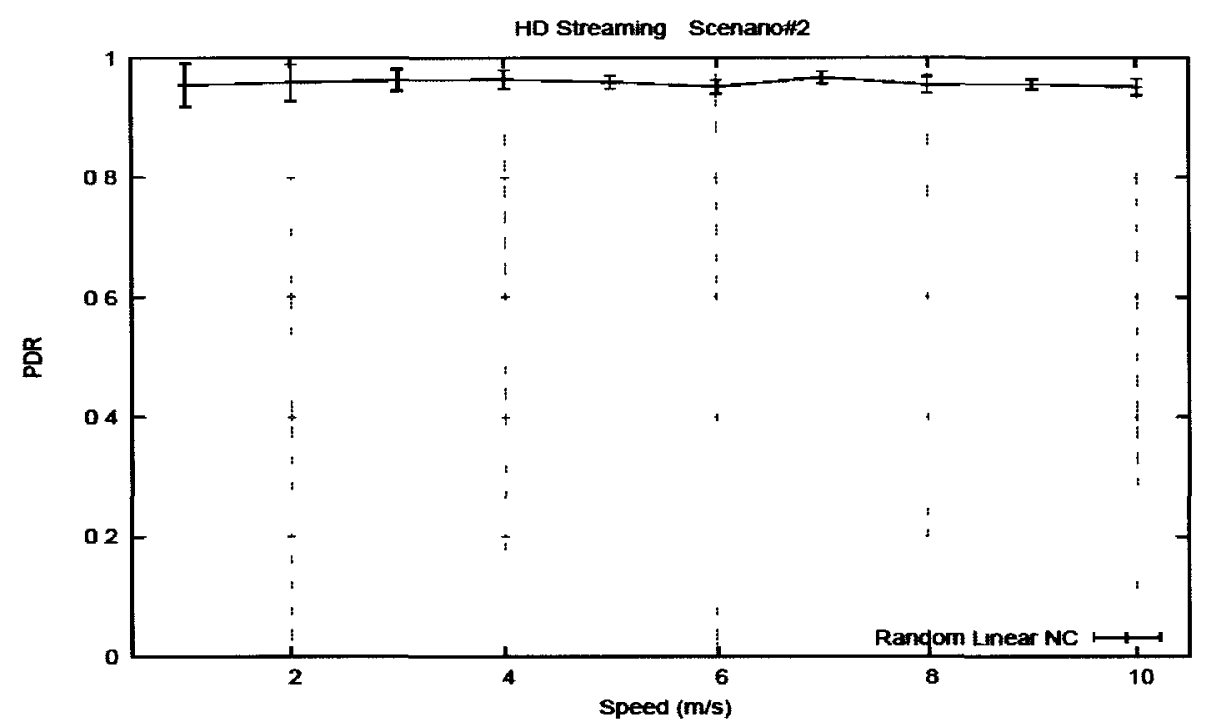

Figure 6.17: PDR for HDTV (Mobile Scenario - Low Density) 
Similarly, Figure 6.15, Figure 6.16 and Figure 6.17 show that the PDR results for video streaming, SDTV and HDTV applications (which have higher data rate and large picket size) are not impacted and remain maintaining steady and high PDR (greater than $90 \%)$

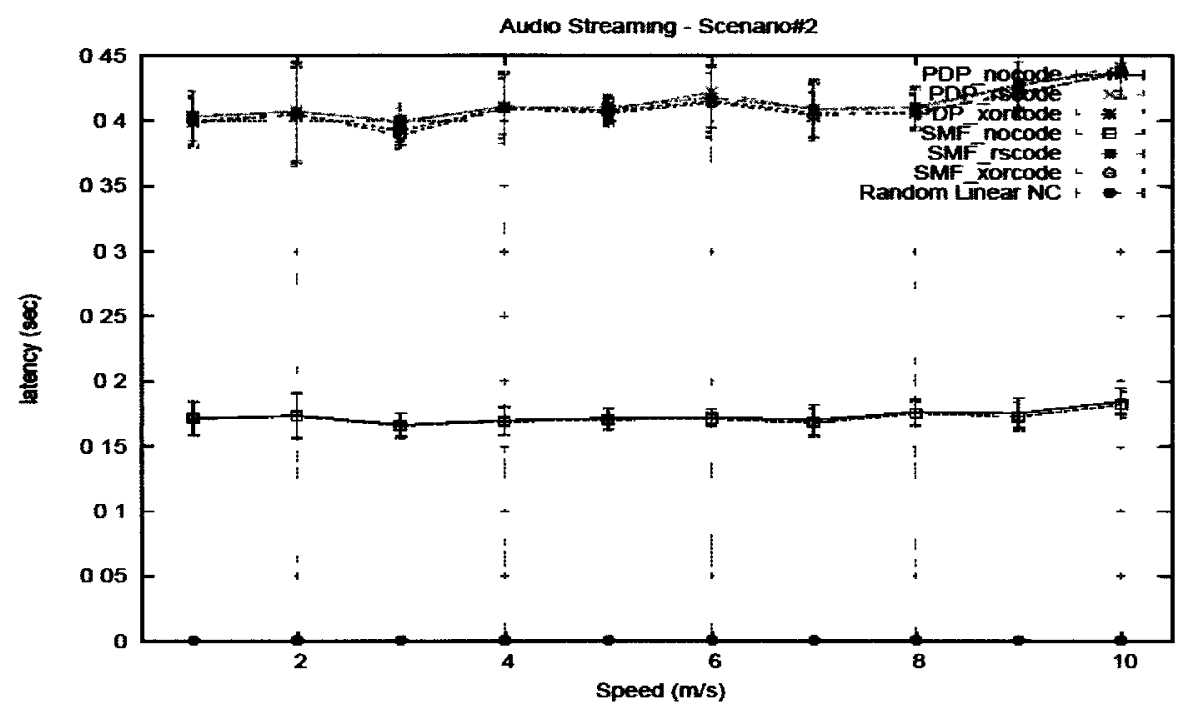

Figure 6.18: Latency for Audio Streaming (Mobile Scenario - Low Density)

Figure 6.18, Figure 6.19, Figure and Figure 8 show the latency results for Scenario\#2. From Figure 6.18, it is clear that the latency for RLNC protocol is the lowest and has an accepted value (below 200ms) for audio streaming applications. The latency values of the tested protocols are not affected by the speed of the wireless nodes. Using RLNC protocol, the sender (relays or BSs) encodes and mixes a large number of packets into one encoded packet and delivers it to the receiving node in a shorter delivery time. However, the latency results of PDP and SMF protocols without using NC techniques are much higher than the RLNC protocol's latency (but below $200 \mathrm{~ms}$ ). The reason of this result is that the packet is queued in different intermediate nodes (relays), or forwarded to 
different nodes; all these factors increase the latency. Due to the previous reasons and the time taken for encoding and decoding packets, the latency of SMF and PDP protocols with $\mathrm{NC}$ techniques are not accepted for audio streaming applications as they are higher than $200 \mathrm{~ms}$.

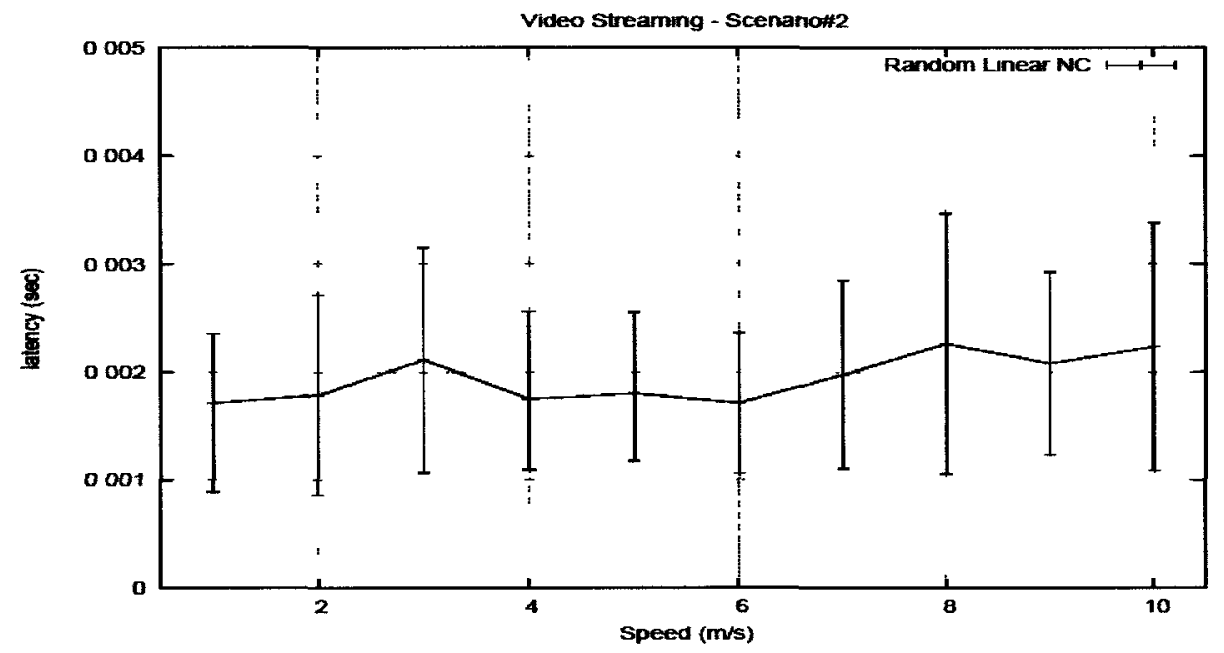

Figure 6.19: Latency for Video Streaming (Mobile Scenario - Low Density)

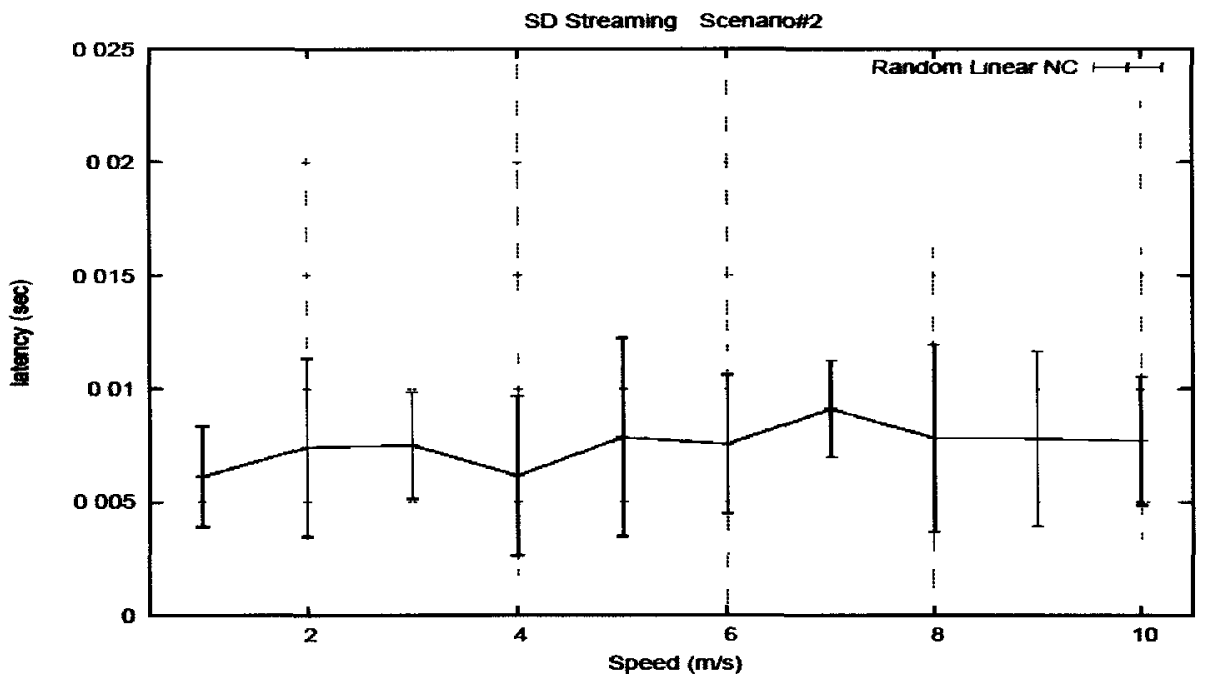

Figure 6.20: Latency for SDTV (Mobile Scenario - Low Density) 


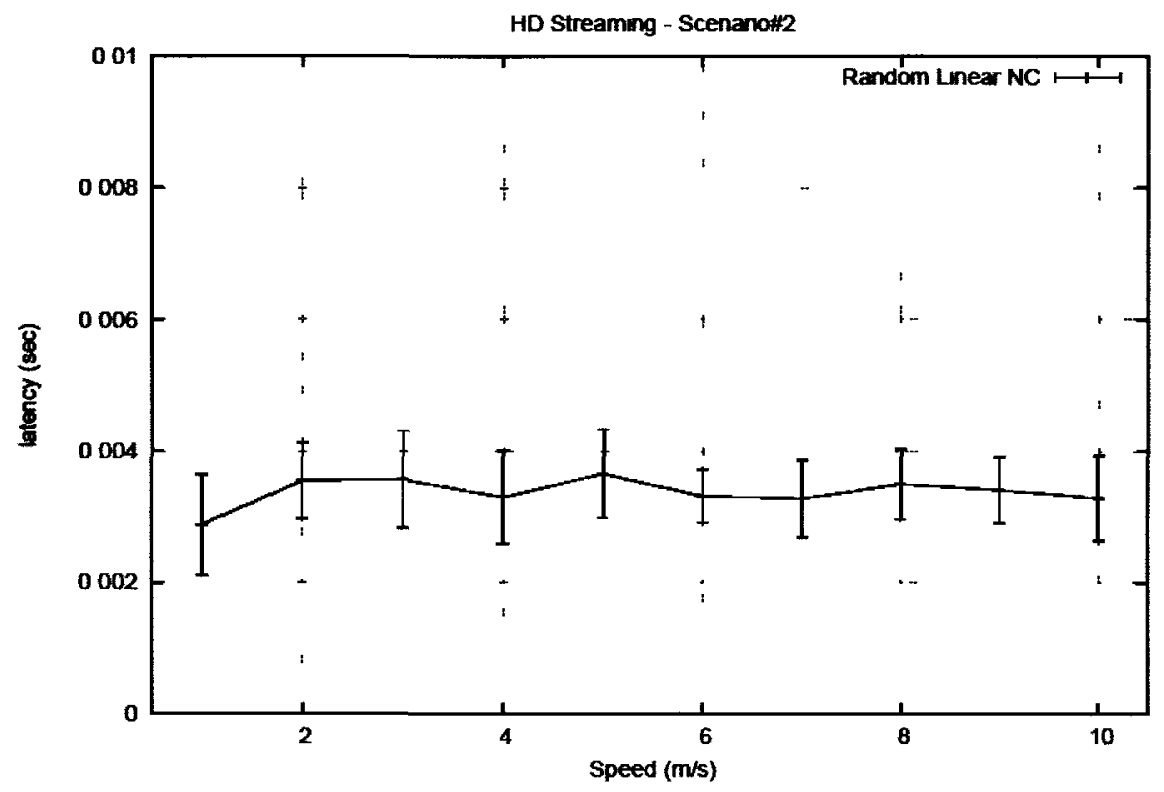

Figure 6.21: Latency for HDTV (Mobile Scenario - Low Density)

Similarly, Figure 6.19, Figure 6.20 and Figure 6.21 show that the latency results for video streaming, SDTV and HDTV applications (which have higher data rate and large picket size) remain below the acceptable value of the latency for multimedia applications (below $200 \mathrm{~ms}$ ).

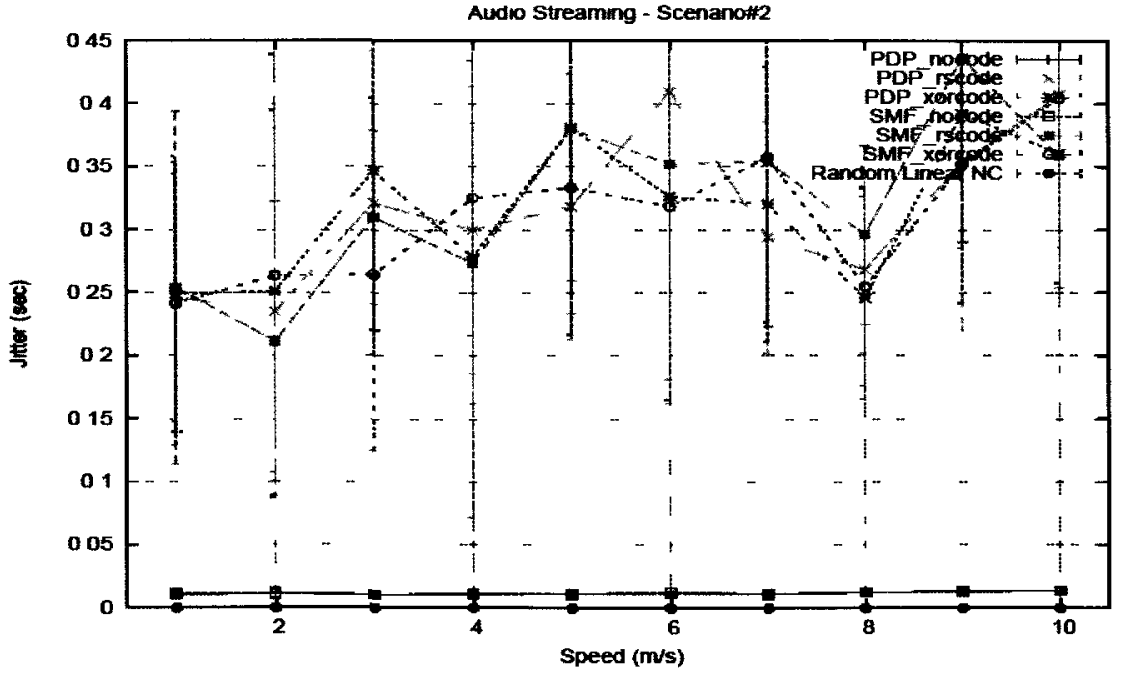

Figure 6.22: Jitter for Audio Streaming (Mobile Scenario - Low Density) 
Figure 6.22, Figure 6.23, Figure 6.24 and Figure 6.25 show the jitter results for scenario\#2 for different multimedia broadcast applications. From this result, it is clear that the jitter value of RLNC is very low. On the other hand, the jitter values of all tested protocols increases by increasing the speed. When the speed increases, the wireless nodes in the network change their locations faster. This leads to increase the variance of the delivery time of the packets, as every time receivers received packets from different neighbor.

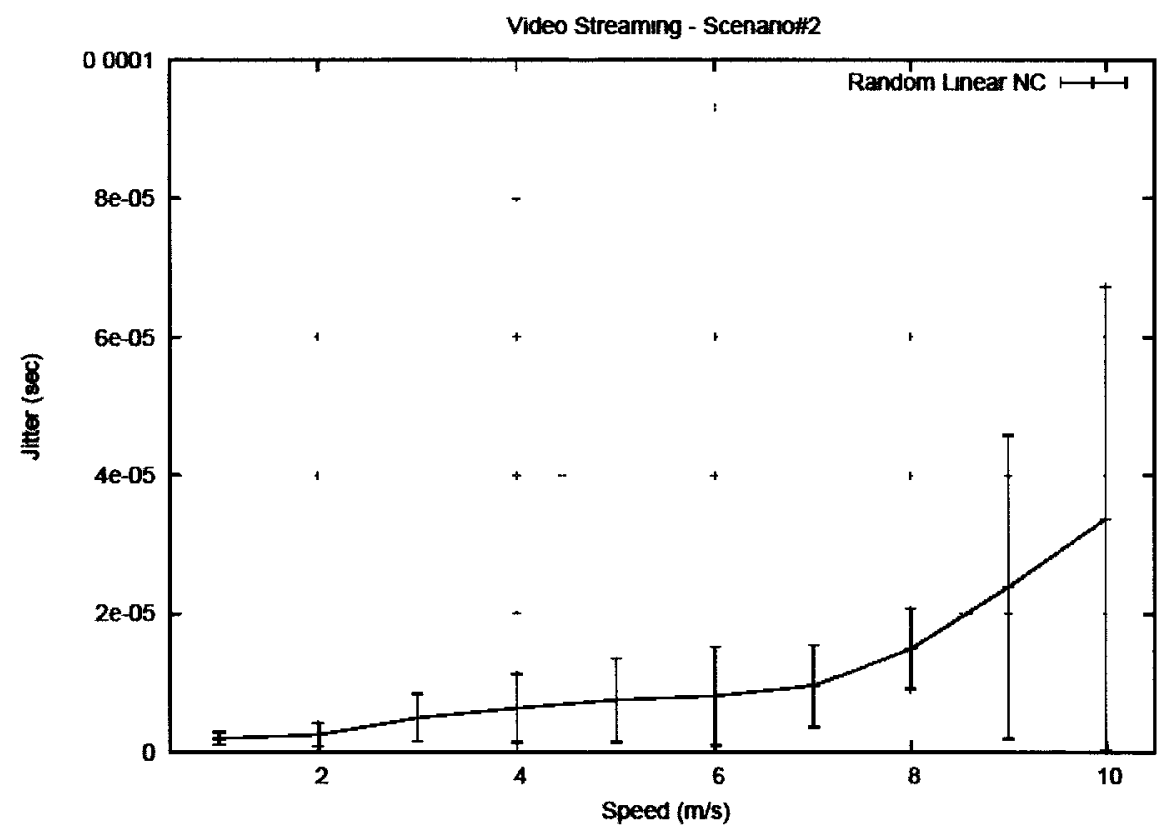

Figure 6.23: Jitter for Video Streaming (Mobile Scenario - Low Density) 


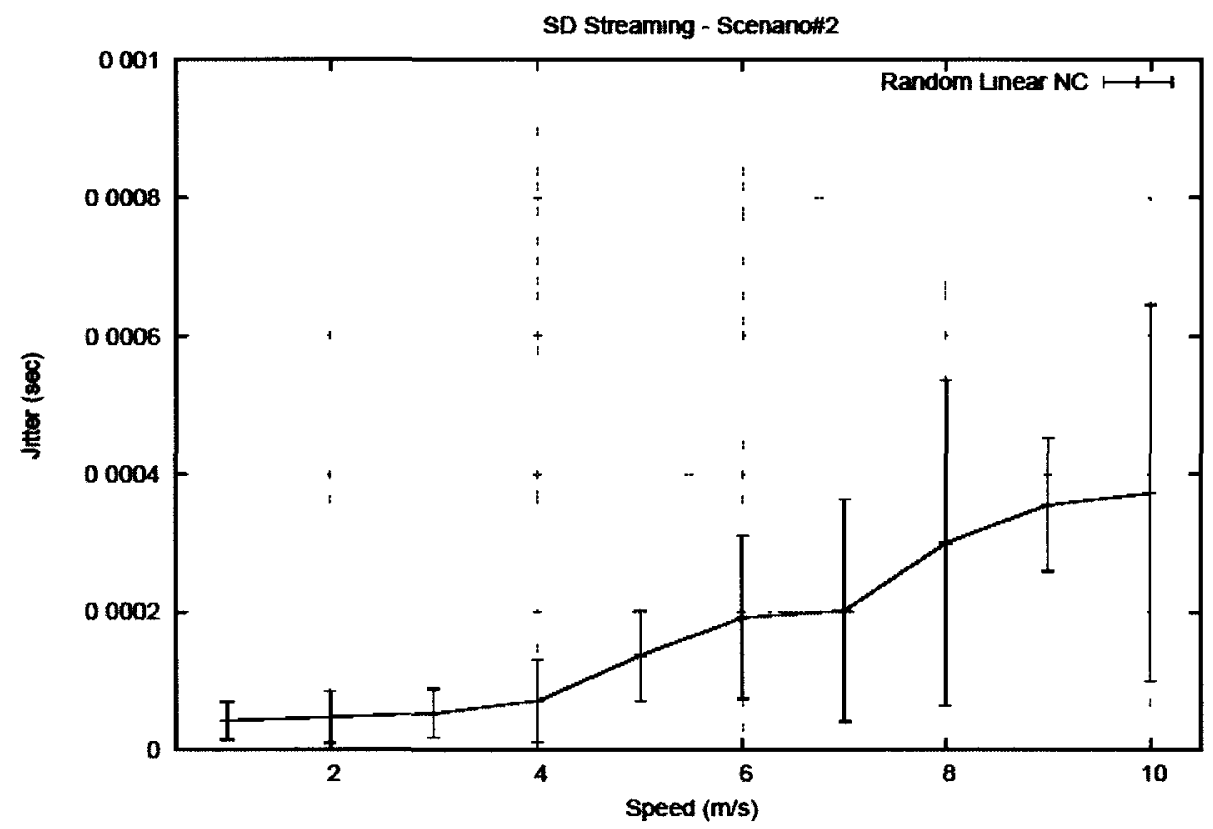

Figure 6.24: Jitter for SDTV (Mobile Scenario - Low Density)

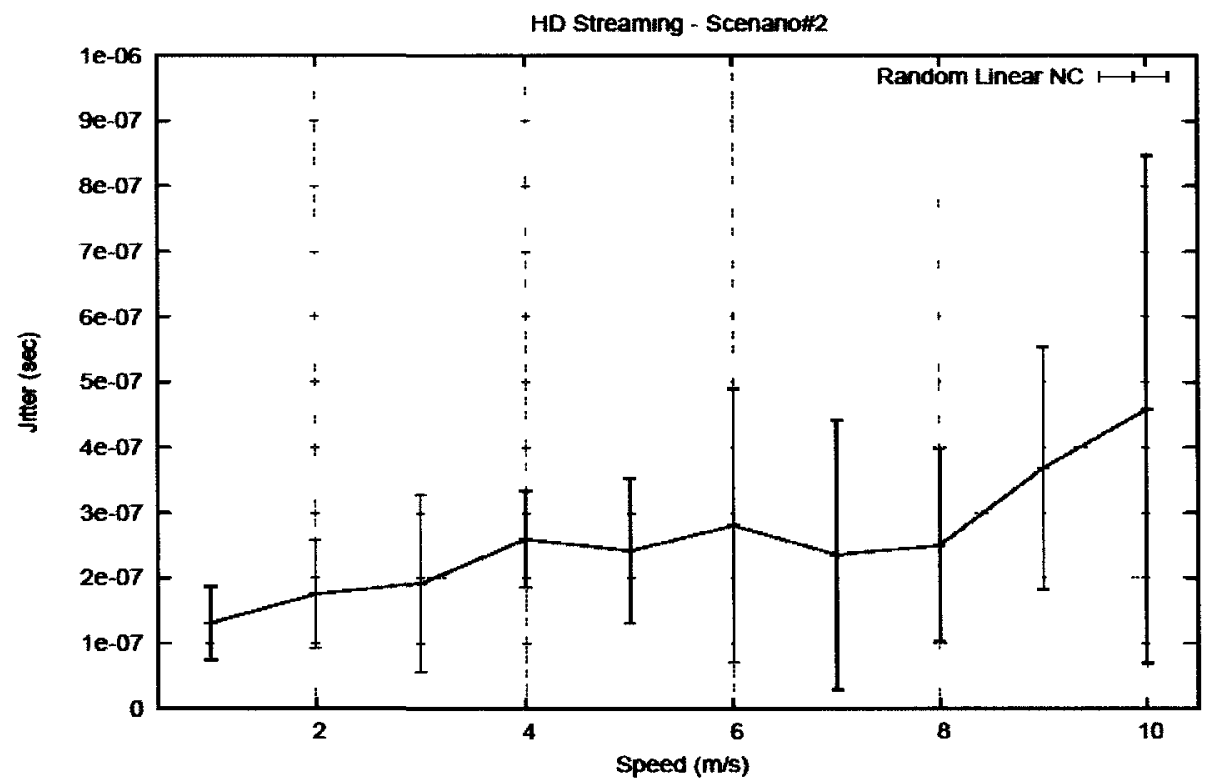

Figure 6.25: Jitter for HDTV (Mobile Scenario - Low Density) 
Based on the previous results, we conclude that the RLNC protocol remains with a good network performance with several multimedia applications; it has the highest value of PDR and the lowest values of latency and jitter.

\subsubsection{0-Wireless-Node Scenarios}

Second set of simulations were established with hundred wireless nodes (high density nodes) placed around BS(s) in order to evaluate the performance difference of several multimedia applications measured at each wireless node over the wireless network. In other words, this set of simulations tests the tested protocols with high density static and mobile nodes. The second set of simulations is divided into two sections; static scenarios and mobility scenarios.

\subsubsection{Static Scenarios}

The following figures contain results obtained from different simulations with different multimedia broadcast applications (Video Streaming, Voice Streaming, SDTV and HDTV traffic) and with varying number of BSs. Since each simulation repeated ten times with different location of the wireless nodes, as a result average of PDR, latency and jitter are shown in the following figures with their confidence intervals. The results for video streaming, SDTV, and HDTV applications will be only tested with RLNC as we proved that the best protocol to be used for multimedia broadcast applications is the RLNC in 6.3.1.1. 


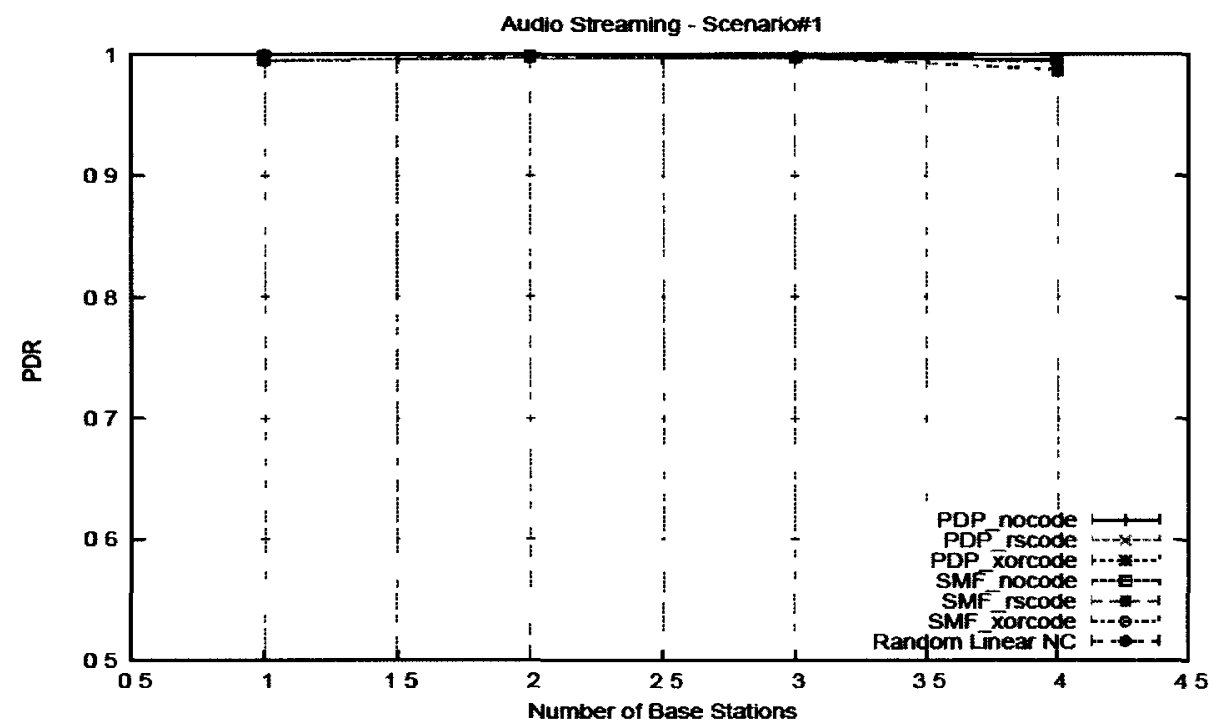

Figure 6.26: PDR for Audio Streaming (Static Scenario - High Density)

Figure 6.26, Figure 6.27, Figure 6.28 and Figure 6.29 show the PDR results for scenario\#1 for different multimedia broadcast applications. By increasing the node density from ten to hundred, all tested protocols show steady performance and there is no effect on the performance of the protocols (above 95\%) for audio streaming applications as shown in Figure 6.26. PDP and SMF protocols without NC are able to control the number of retransmissions by neighbor knowledge as a result there is no impact observed by increasing the node density. Adding to the previous reason, PDP and SMF protocols with NC have higher chances to receive information needed to decoded encoded received packets from the wireless nodes. As mentioned in 6.3.1.1, the RLNC protocol is not mainly affected because the RLNC protocol depends on the coefficients rather than the overhearing opportunity which are added in the headers of the encoded packets during the encoding procedure to decode the encoded packets. 


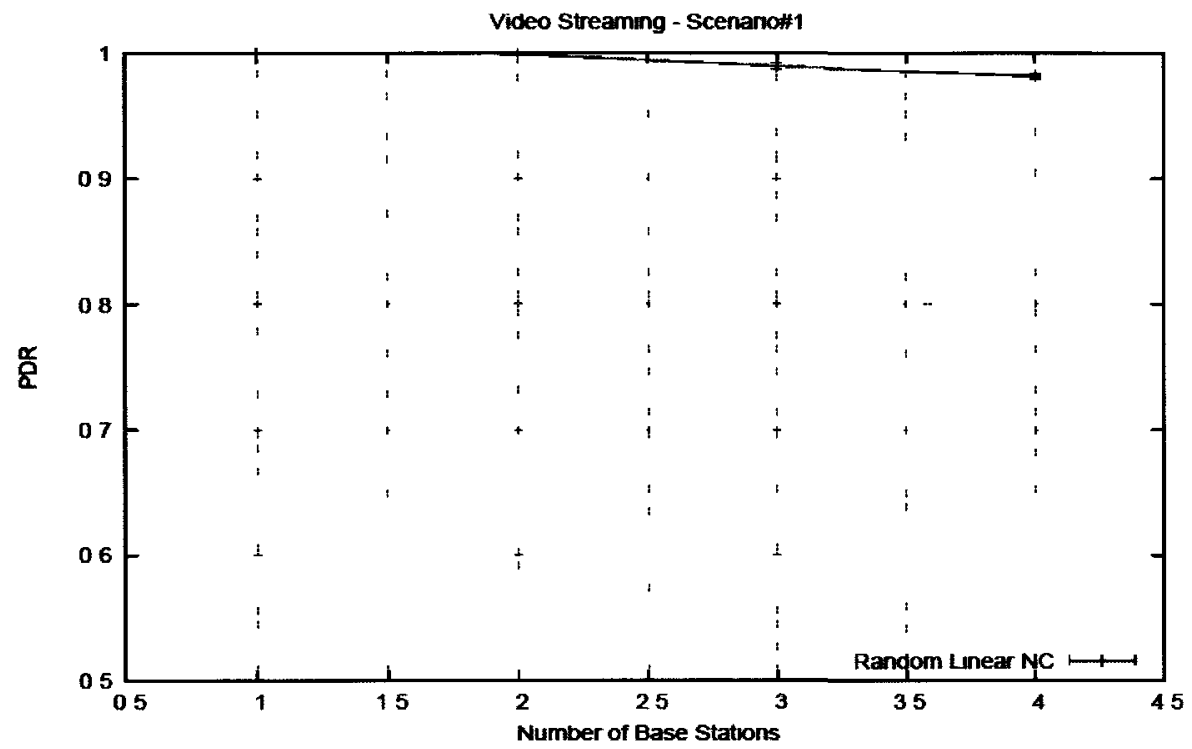

Figure 6.27: PDR for Video Streaming (Static Scenario - High Density)

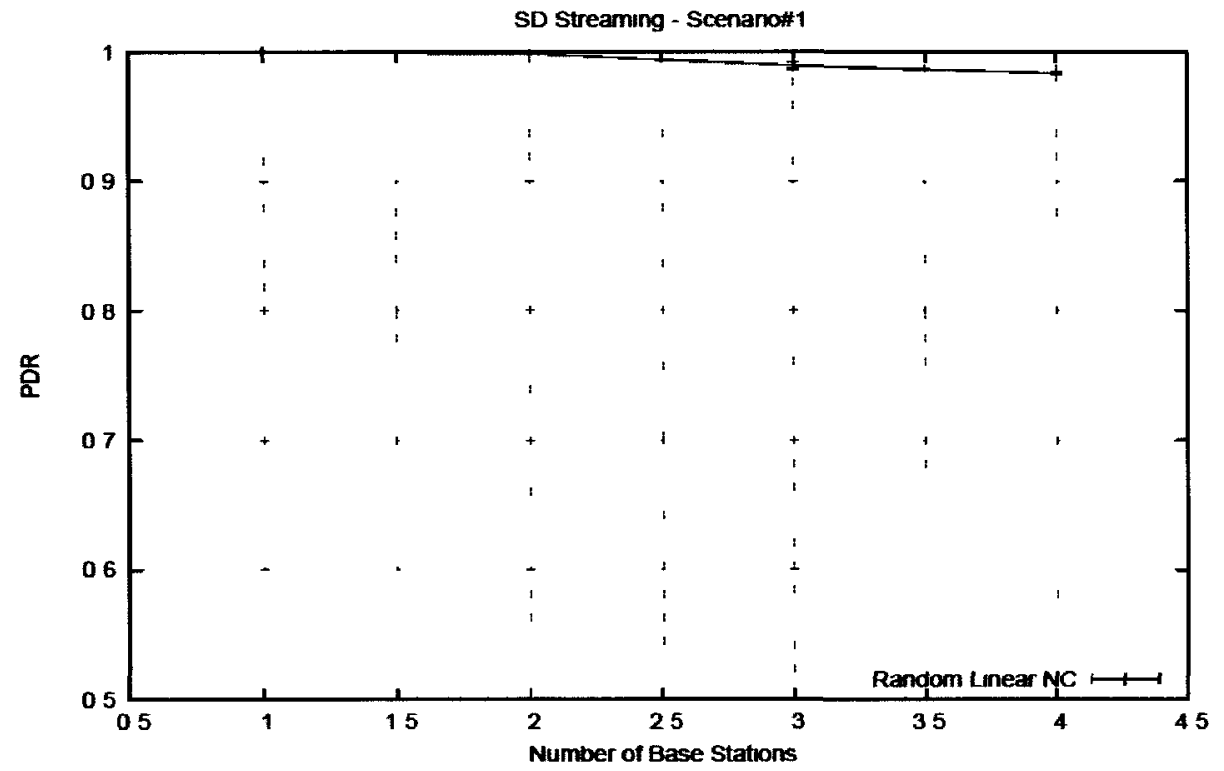

Figure 6.27: PDR for SDTV (Static Scenario - High Density) 


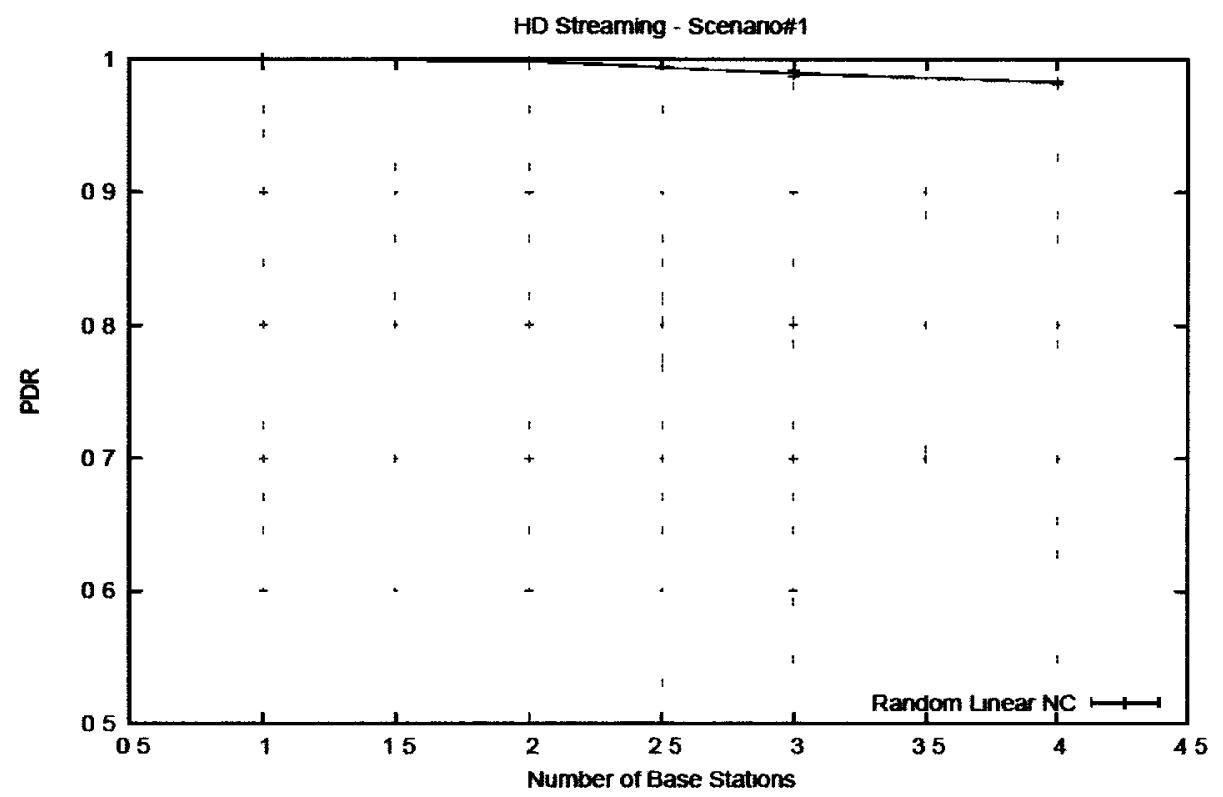

Figure 6.29: PDR for HDTV (Static Scenario - High Density)

Moreover, RLNC protocol performs very well in video streaming, SDTV and HDTV applications as shown in Figure 6.27, Figure 6.28 and Figure 6.29. The figures show that the PDR results of RLNC start slowly dropping with multimedia broadcast applications that have large packet sizes and higher data rates when number of BSs increases in a high density environment. The reason of this is that the wireless channel is always busy and many packets do not get the chance to be transmitted and are dropped at BS in high density node networks. However, the PDR of RLNC remains high and acceptable for multimedia broadcast applications.

Figure 6.30, Figure 6.31, Figure 6.32 and Figure 6.33 show the latency results for Scenario\#1 for several multimedia broadcast applications. As shown in Figure 6.30, in audio streaming applications the latency values for hundred wireless nodes networks are 
higher compared to the latency value for the same scenario of ten wireless nodes networks. However, the latency result of RLNC remains the lowest compared to all other latency values and is acceptable to audio streaming applications. The latency results of SMF and PDP with/without NC are high and not acceptable values for the audio streaming applications as they are higher than $200 \mathrm{~ms}$. The reason of this result is that in SMF and PDP without NC protocols the number of flooded packets in the high density nodes networks increases, which increases the queue size at the receiver. As a result of this, the latency increases. Moreover, in SMF and PDP with NC the latency is affected by two factors; the large size of the queue which increases in high density networks and the long needed time to encode and decode packets (number of encoded received packets will increase when number of wireless nodes increases). Both factors increase the overall latencies of both SMF and PDP with NC.

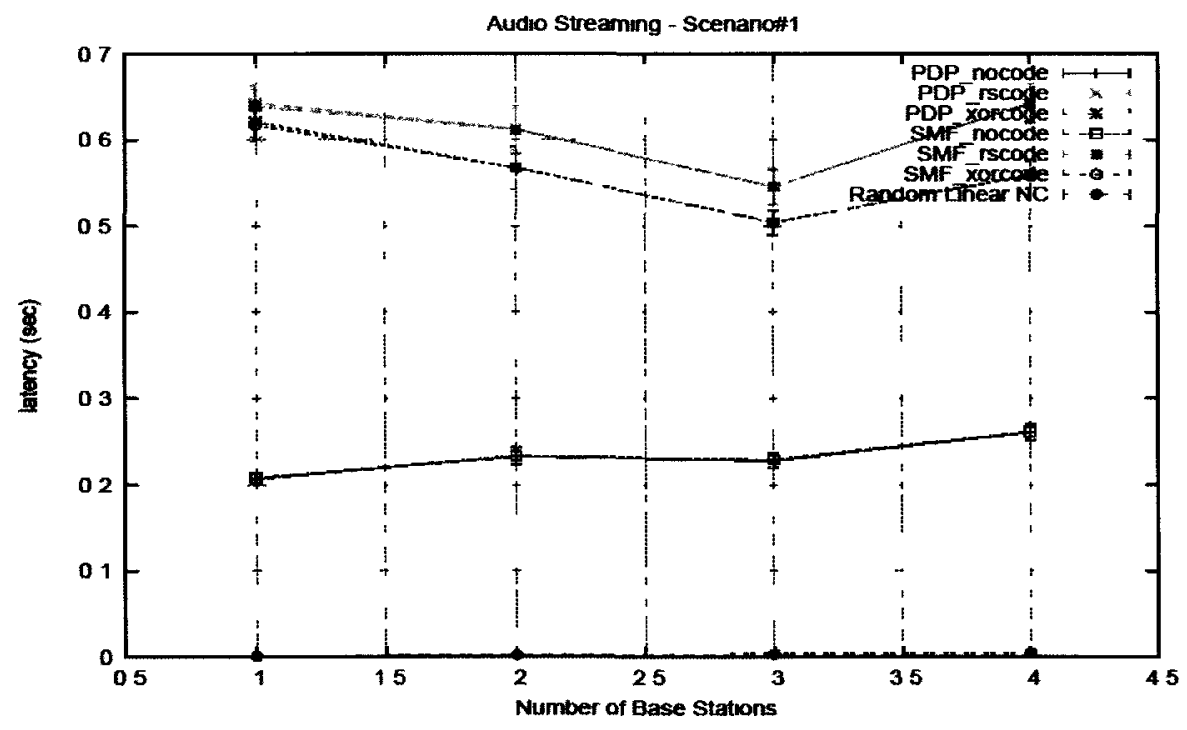

Figure 6.30: Latency for Audio Streaming (Static Scenario - High Density) 
Similarly, the latency results of RLNC remain as an acceptable value for video streaming, SDTV and HDTV applications. However, the figures show that the latency results linearly increases as number of BSs increases. The reason of this is that the by increasing number of BSs in high density networks, number of broadcasted packets will increases. Therefore, the needed time to deliver each packet to all wireless nodes will increase. Also, the latency values for hundred wireless node networks are higher compared to the latency values for the same scenario of ten wireless node networks. The reason of this is that number of encoded packets which are travelling in the network will increase, as number of wireless nodes (act like routers and have the ability to encode and decode packets) in the network increases. This lead to increase the overall latency in the network as the procedure of encoding and decoding requires more time.

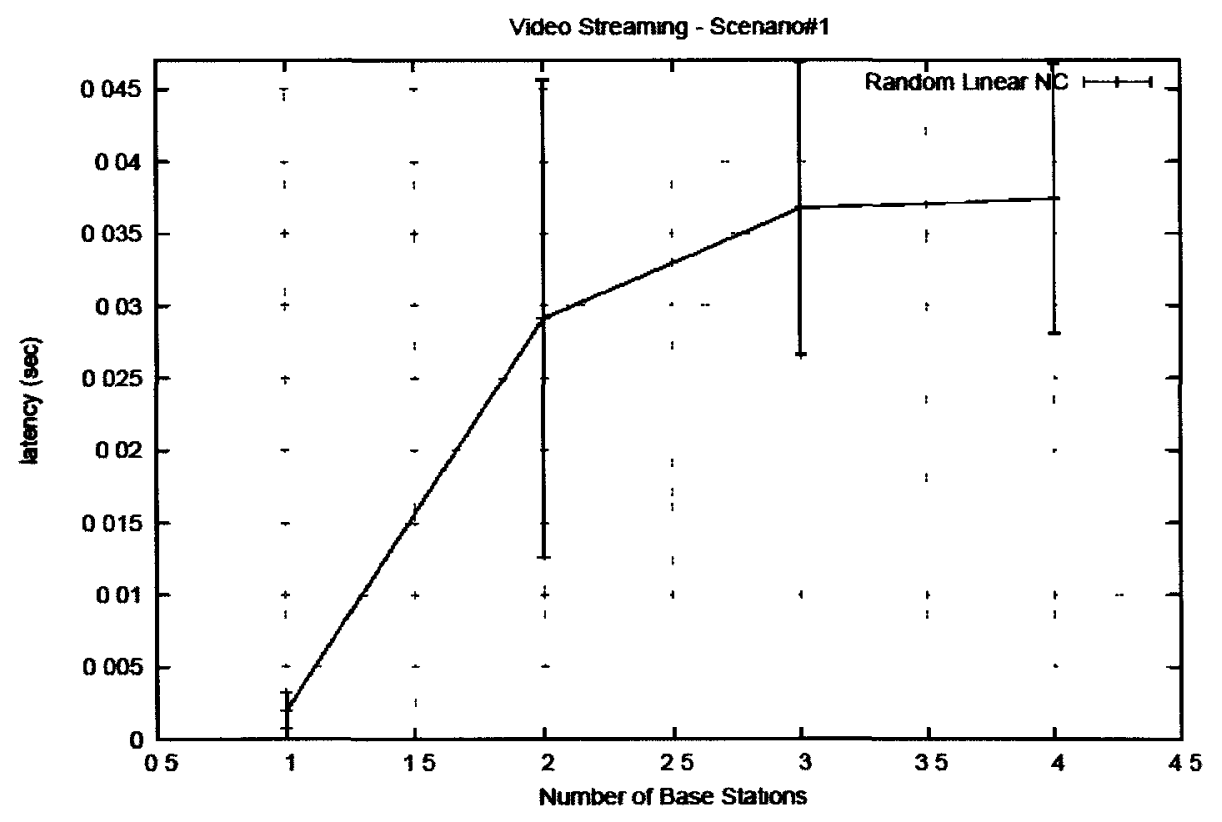

Figure 6.31: Latency for Video Streaming (Static Scenario - High Density) 


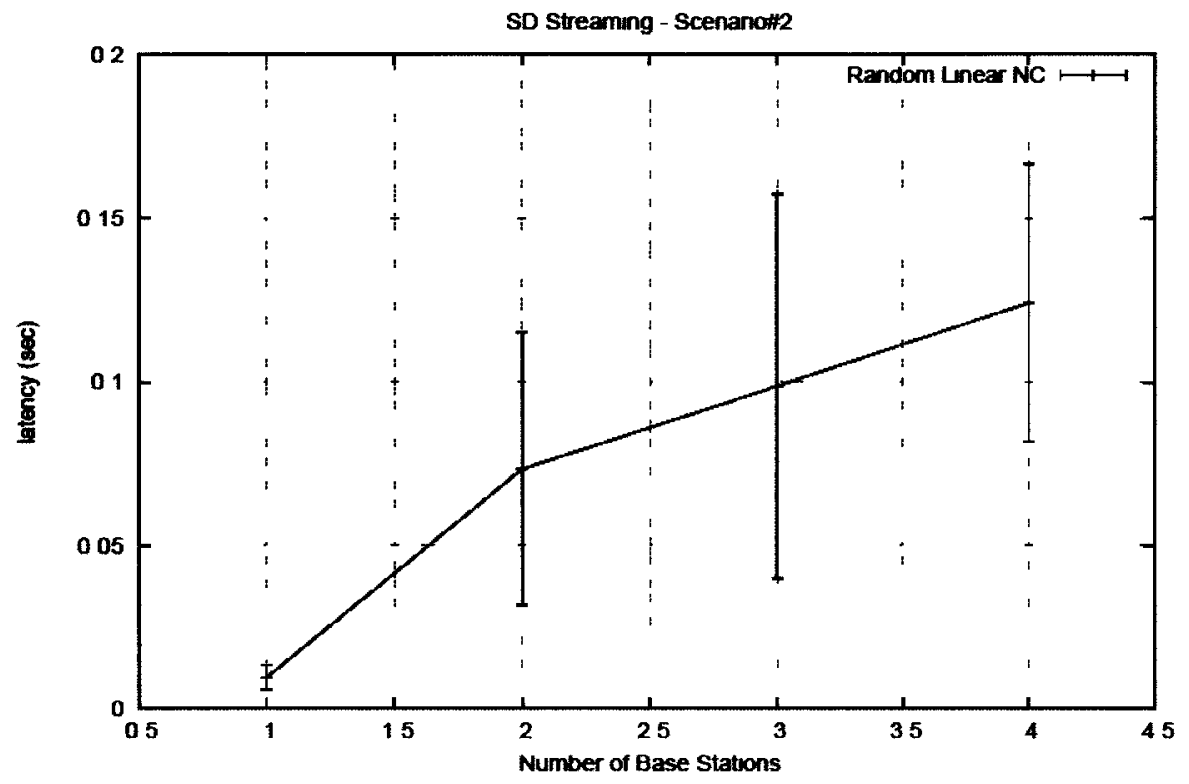

Figure 6.32: Latency for SDTV (Static Scenario - High Density)

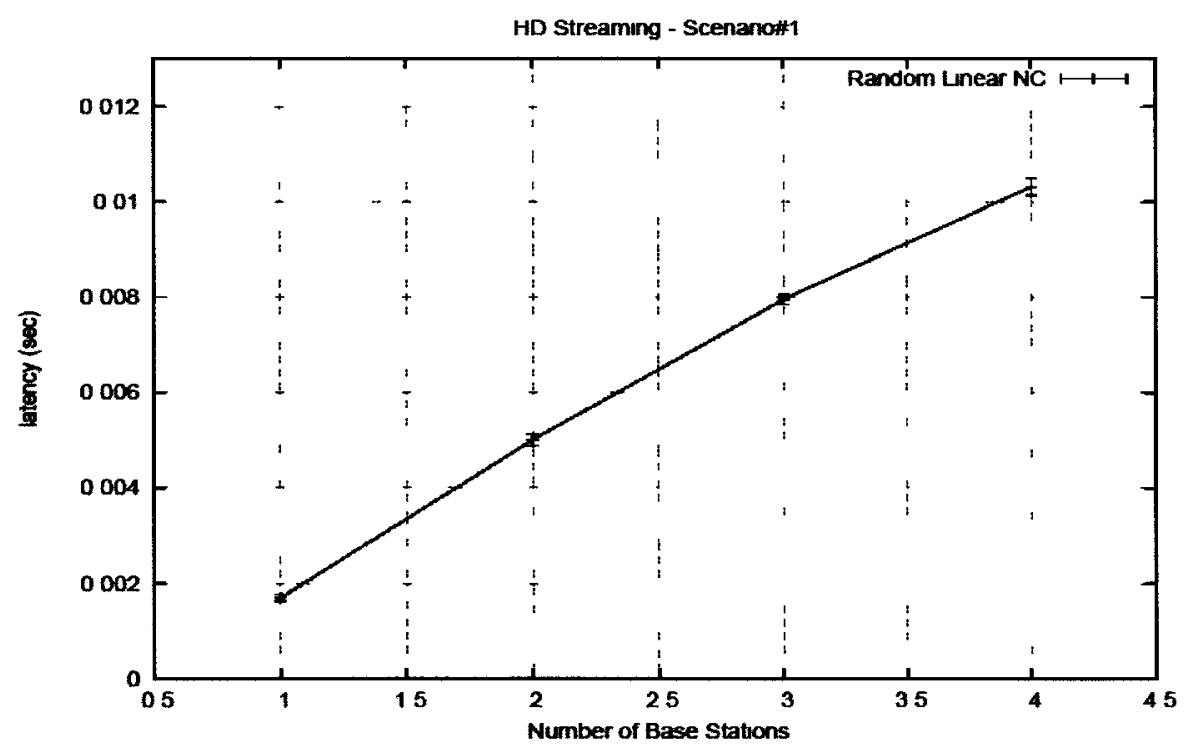

Figure 6.33: Latency for HDTV (Static Scenario - High Density) 
Figure 6.34, Figure 6.35, Figure 6.36 and Figure 6.37 show the jitter results for scenario\#1 for different multimedia broadcast applications. Excluding PDP-XOR and SMF-XOR, Figure 6.34 shows that number of wireless nodes does not impact the jitter values as the values of the jitter in high density networks are almost the same of the jitter values of low density networks. In general, it is clear that the jitter values of SMF and PDP with NC are the highest compared to the other jitter values. The reason of this result is due to the dependency on the opportunistic listening mechanism which impacts the differences of the latency of the received packets, where all receiving nodes wait for overheard packets to successfully decode the received encoded packets. However, PDP and SMF with XOR technique in the high destiny networks have lower jitter values compared to the values in the low density networks. The reason of this is that by increasing the density of the network, the opportunity of delivering information to the receivers increases which leads to decrease the latency (Figure 6.30) and the various times (jitter) of delivering/encoding/decoding packets. On the other hand, RLNC has the lowest jitter in both scenarios as the receiving nodes use the coefficients which are stored in the header to decode the received encoded packets. As a result, this will decrease the jitter of the network compared to the other tested protocols. Moreover, the jitter results of SMF and PDP without NC have also a low value but higher than RLNC. The reason of this result is that some packets may be queued in different intermediate nodes, or forwarded to different nodes; these factors have an impact on the latency which impacts the jitter. 


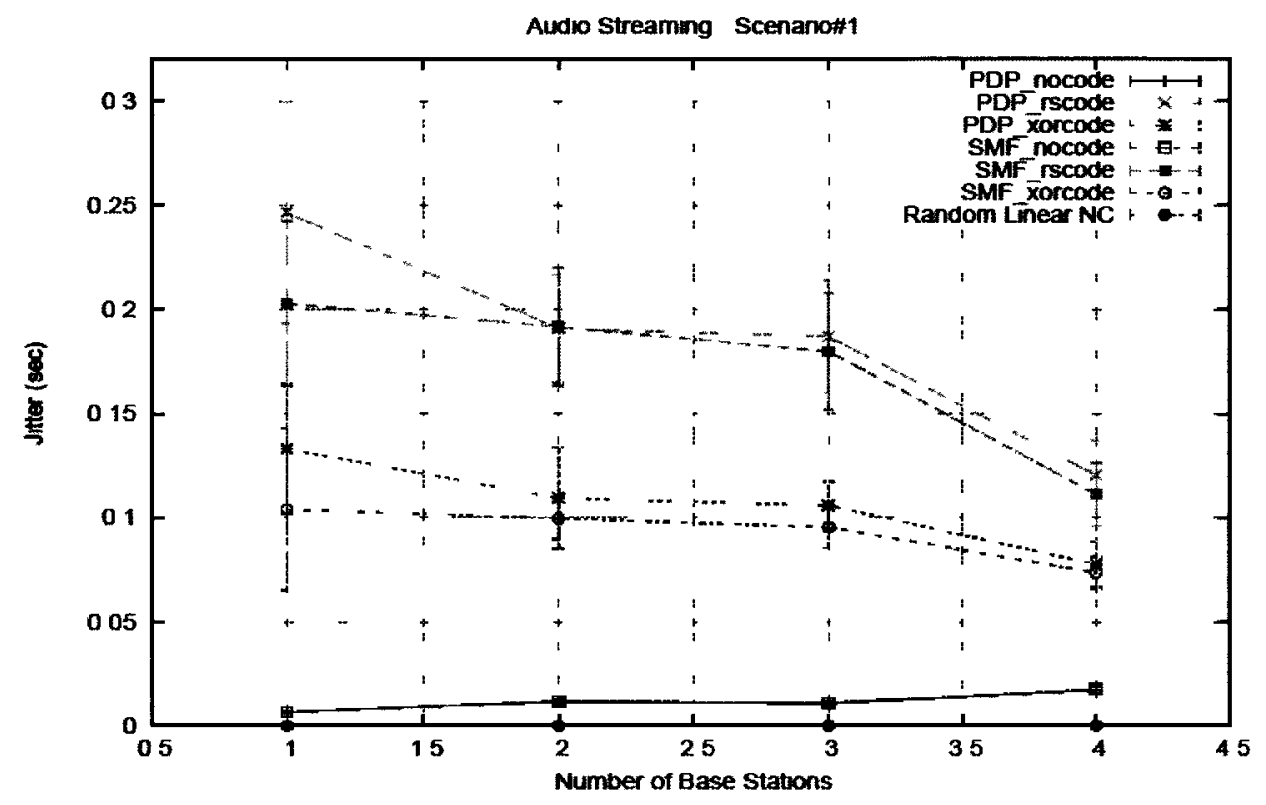

Figure 6.34: Jitter for Audio Streaming (Static Scenario - High Density)

Furthermore, Figure 6.35, Figure 6.36 and Figure 6.37 show the jitter results of RLNC protocols with video streaming, SDTV and HDTV applications. The figures show that the RLNC protocol has a very low jitter value which does not affect the multimedia broadcast applications quality, as known that if the jitter value is high the quality of multimedia broadcast applications contents will get negatively impacted and affected. However, the jitter values of RLNC increase in the high density networks, but as shown in the figures the increase of the jitter values are slightly small which does not hugely affect the quality of the multimedia broadcast applications. 


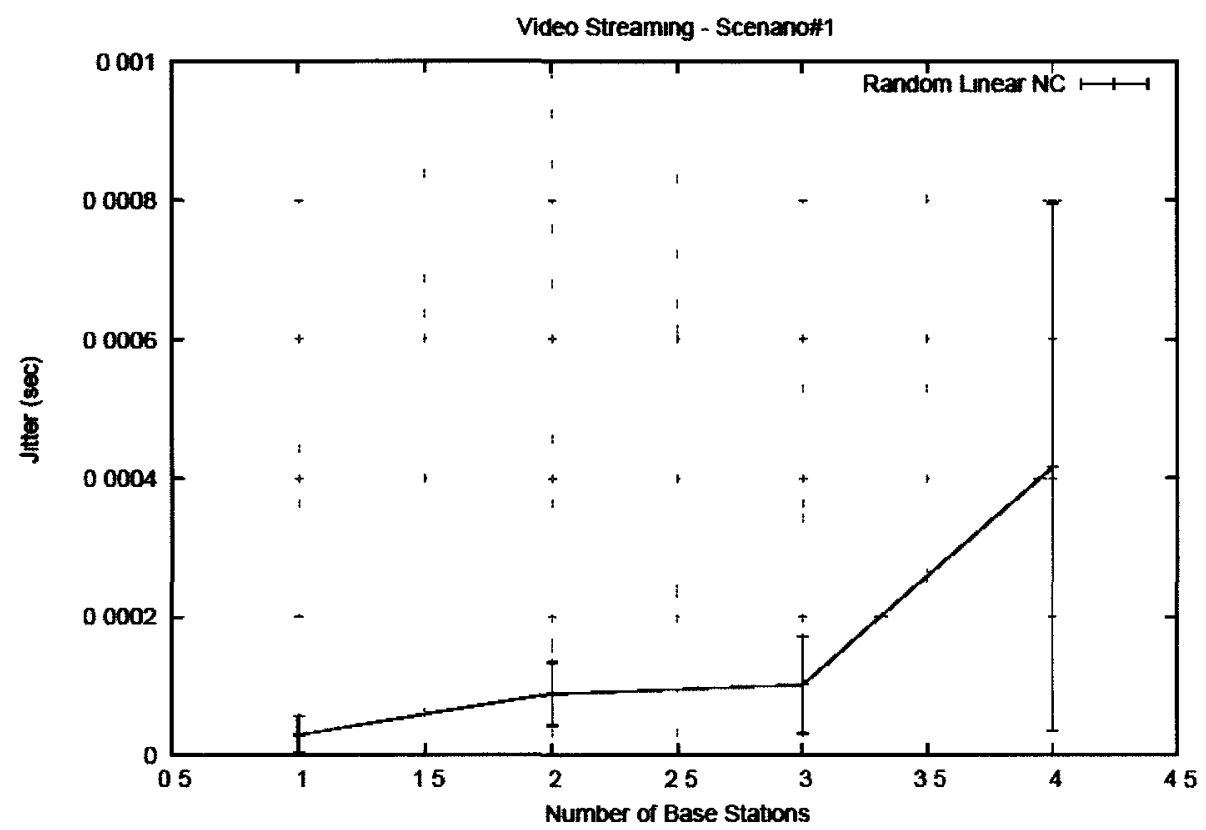

Figure 6.35: Jitter for Video Streaming (Static Scenario - High Density)

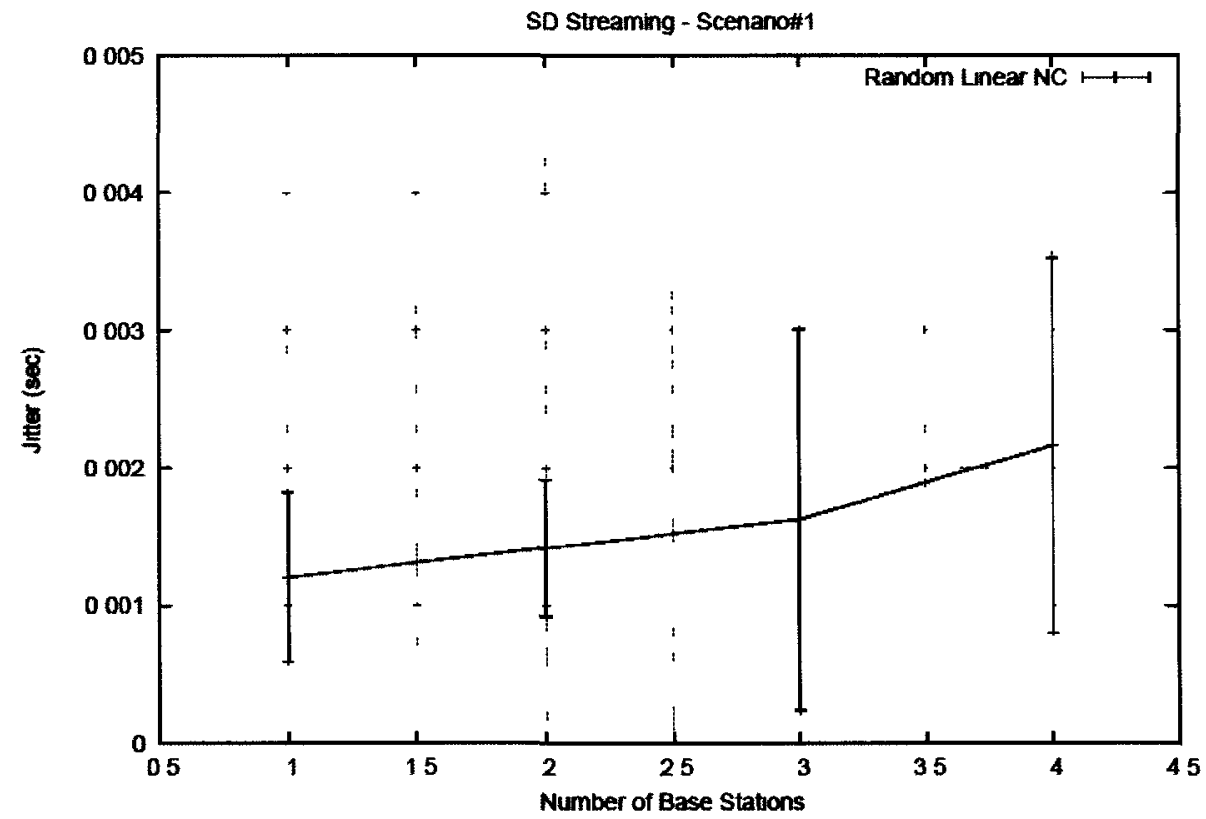

Figure 6.36: Jitter for SDTV (Static Scenario - High Density) 


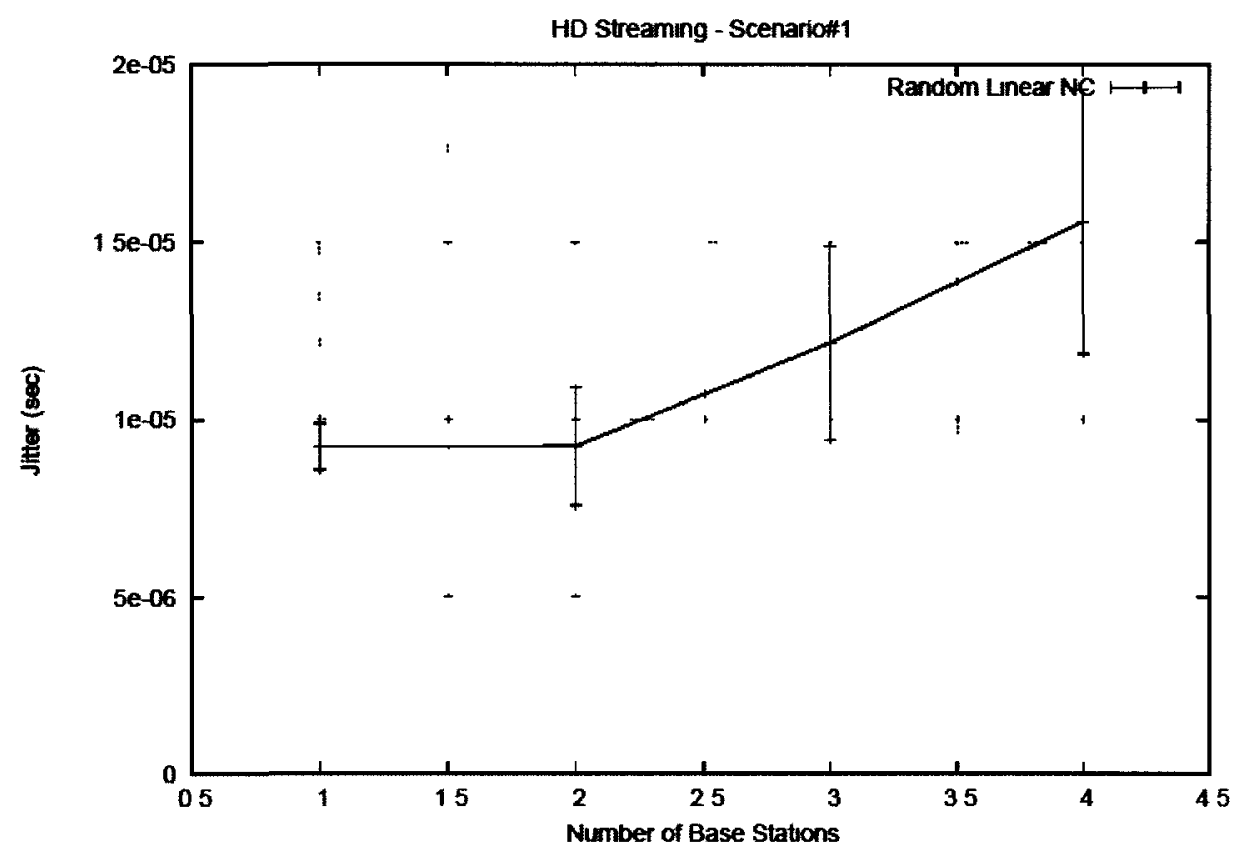

Figure 6.37: Jitter for HDTV (Static Scenario - High Density)

Based on the previous results, the best protocol to be used for multimedia broadcast applications is also the RLNC as it has the highest value of PDR and the lowest values of latency and jitter. As a result, RLNC improves the overall performance of the multımedia broadcast applicatıons in high density networks.

\subsubsection{Mobility Scenarios}

The following figures contain results obtained from different simulations with different multimedia broadcast applications with varyıng number of BSs. Since each simulation repeated ten times with different location of the wireless nodes, as a result average of PDR, latency and jitter are shown in the following figures with their confidence intervals. The results for video streaming, SDTV, and HDTV applications 
will be only tested with RLNC as we proved that the best protocol to be used for multimedia broadcast applications is the RLNC in 6.3.1.1.

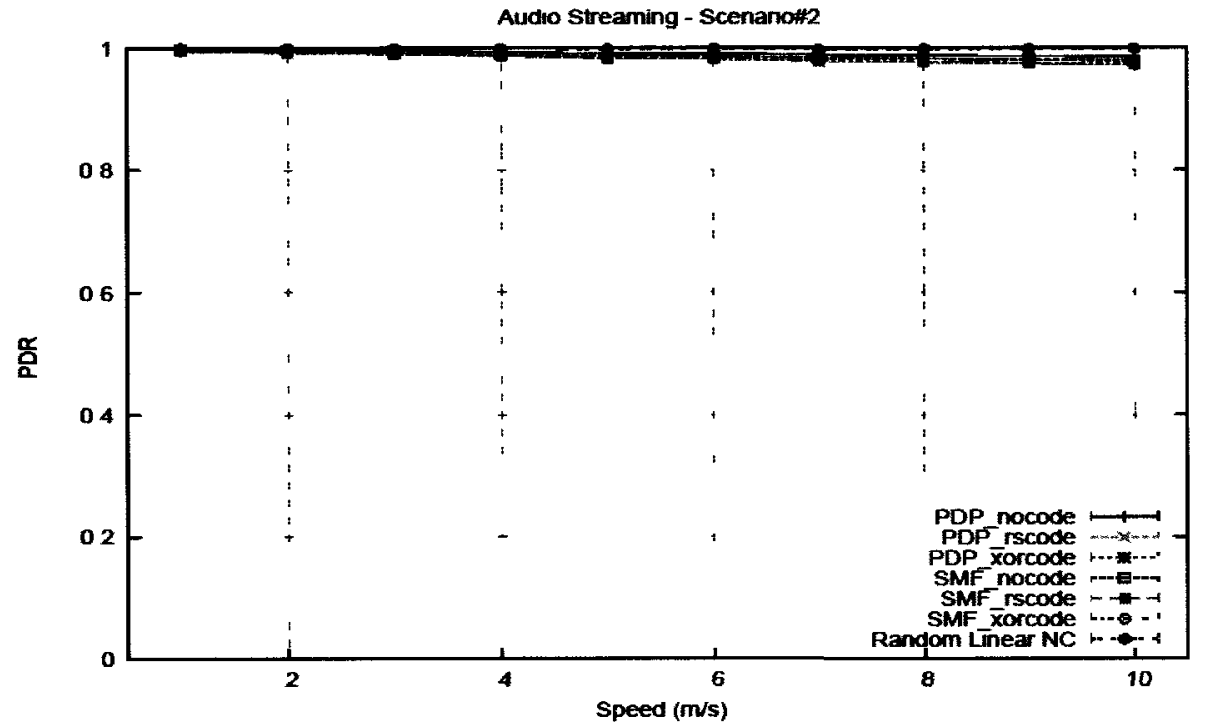

Figure 6.38: PDR for Audio Streaming (Mobile Scenario - High Density)

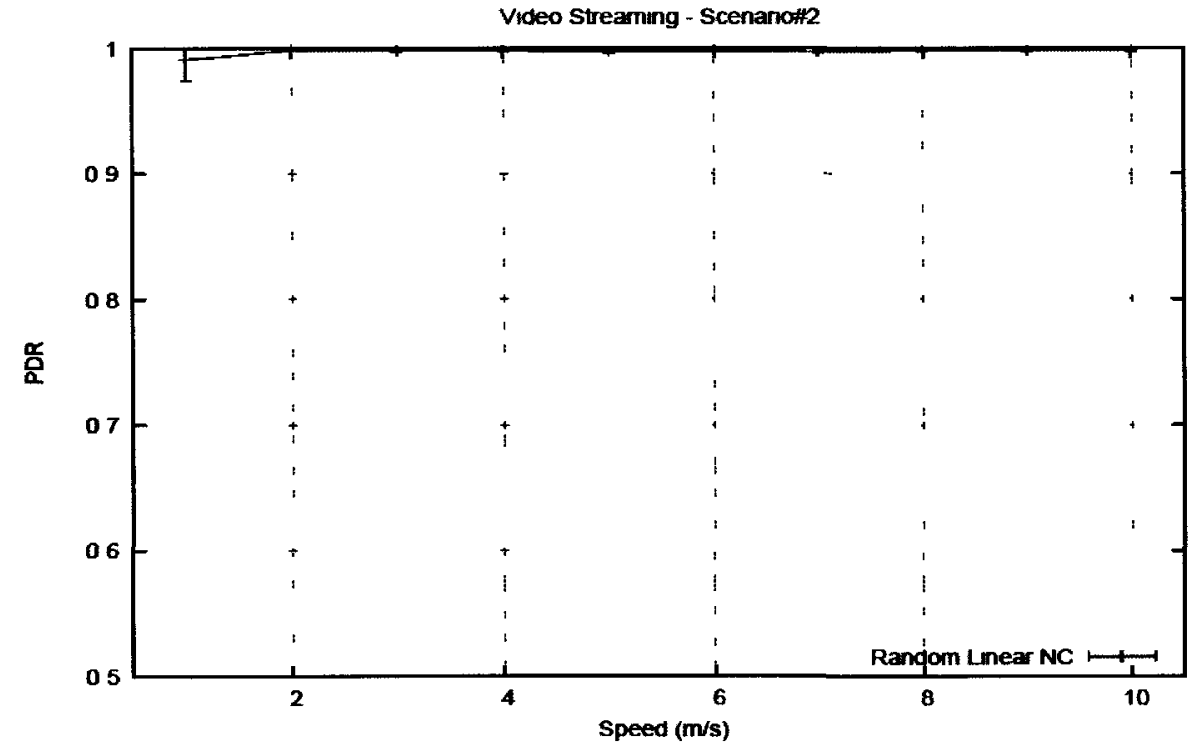

Figure 6.39: PDR for Video Streaming (Mobile Scenario - High Density) 


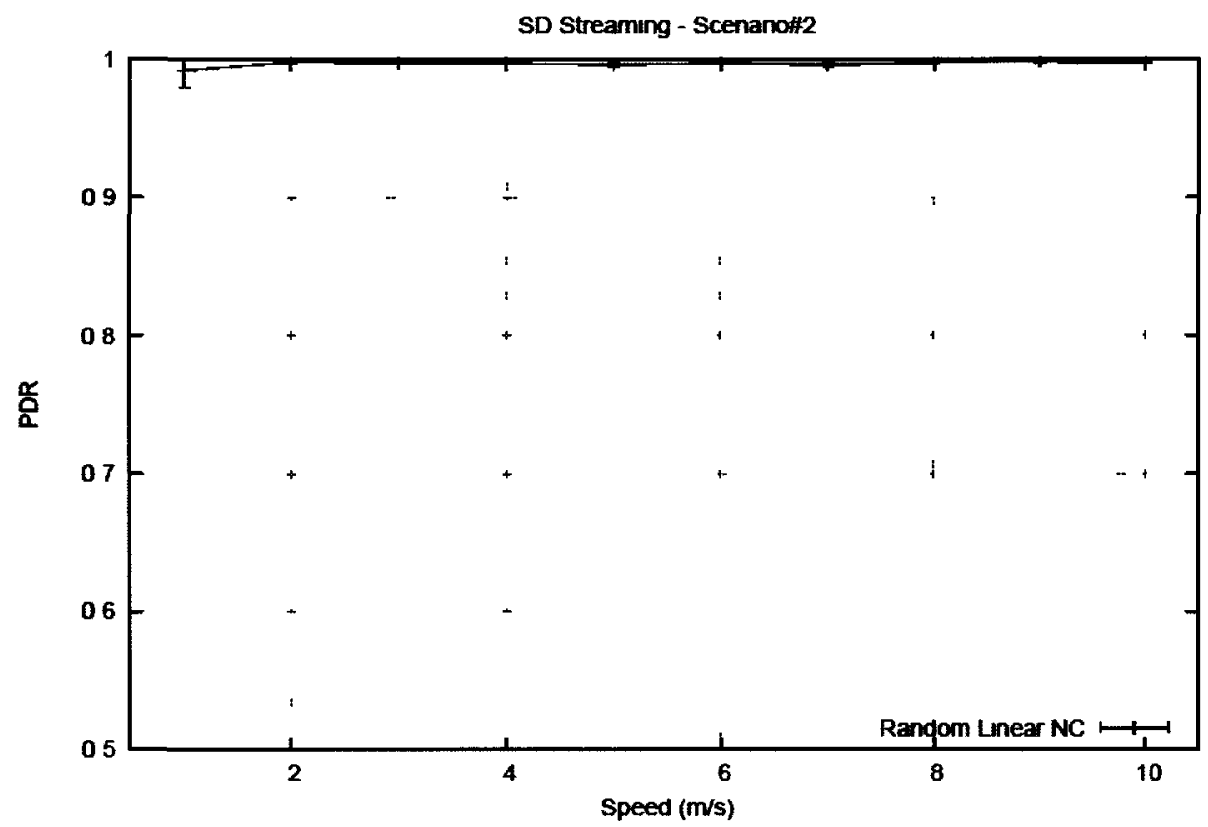

Figure 6.40: PDR for SDTV (Mobile Scenario - High Density)

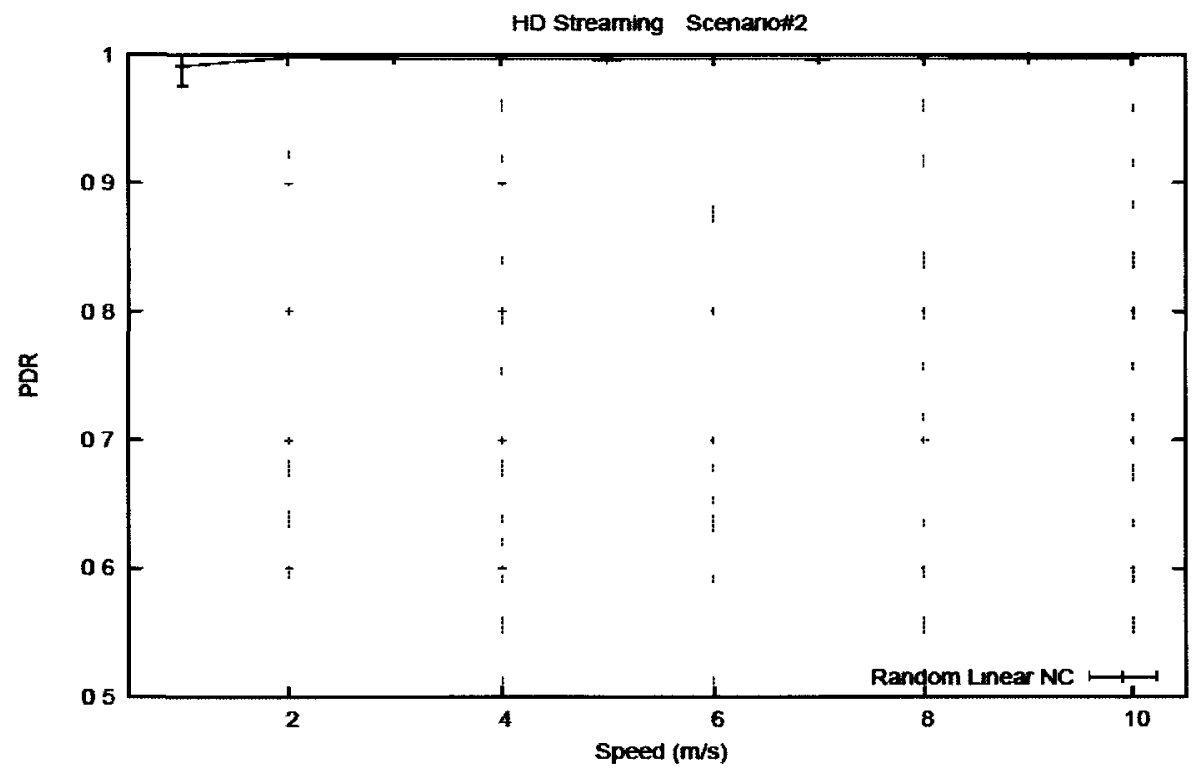

Figure 6.41: PDR for HDTV (Mobile Scenario - High Density) 
Figure 6.38, Figure 6.39, Figure 6.40 and Figure 6.41 show the PDR results for Scenario\#2. From Figure 6.38, the PDR for RLNC protocol is the highest for audio streaming applications. The PDR of RLNC protocol approximately reaches $100 \%$ over various speeds. Also by increasing the node density from ten to hundred, all tested protocols show steady performance and there is no effect on the network performance (above 95\%) for audio streaming applications. Similar to scenario\#1, PDP and SMF protocols without $\mathrm{NC}$ are able to control the number of retransmissions by neighbor knowledge as a result there is no impact observed by varying node densities. Adding to the previous reason, PDP and SMF protocols with NC have higher chances to receive information needed to decoded encoded received packets from the wireless nodes. As mentioned in previous sub-sections, the RLNC protocol is not mainly affected because the RLNC protocol depends on the coefficients rather than the overhearing opportunity which are added in the headers of the encoded packets during the encoding procedure to decode the encoded packets.

Moreover, RLNC protocol have very high PDR values for video streaming, SDTV and HDTV applications as shown in Figure 6.39, Figure 6.40 and Figure 6.41 . The figures show that the PDR of RLNC have steady state in a high density environment over different speeds. 


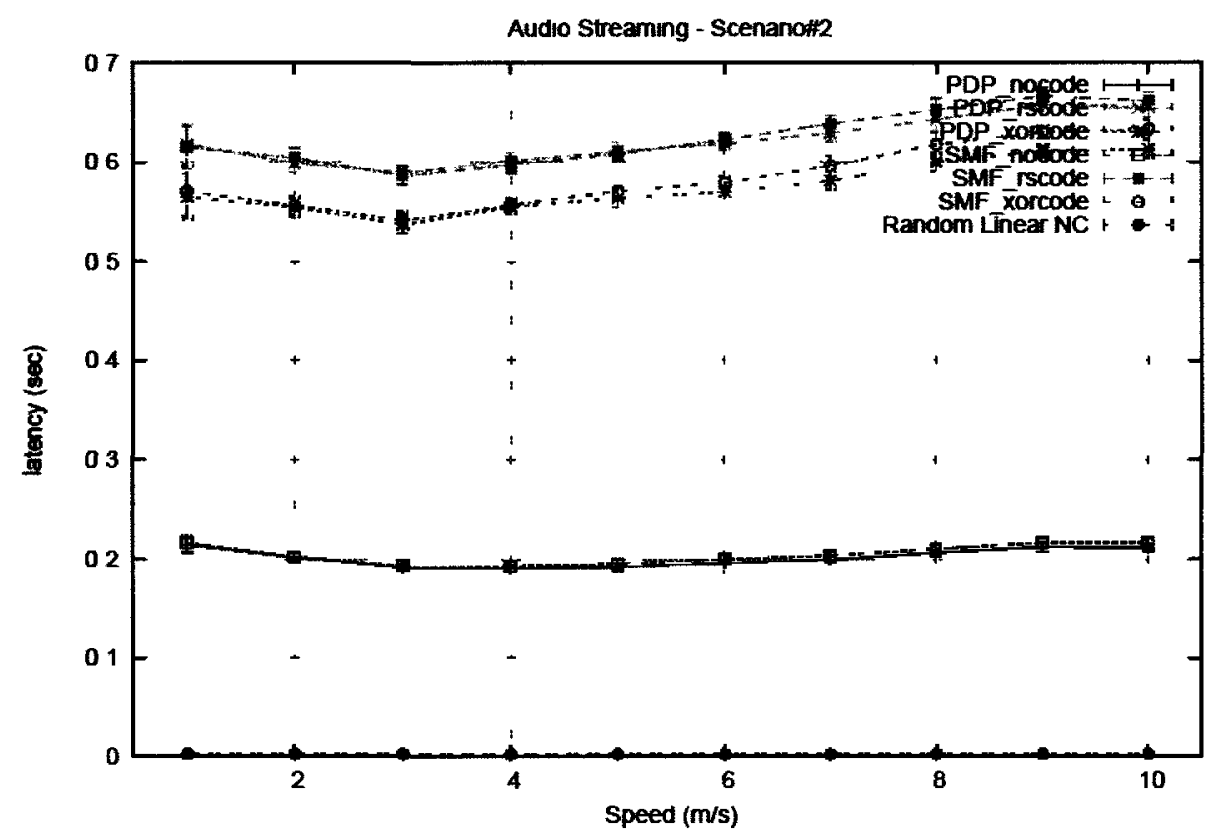

Figure 6.42: Latency for Audio Streaming (Mobile Scenario - High Density)

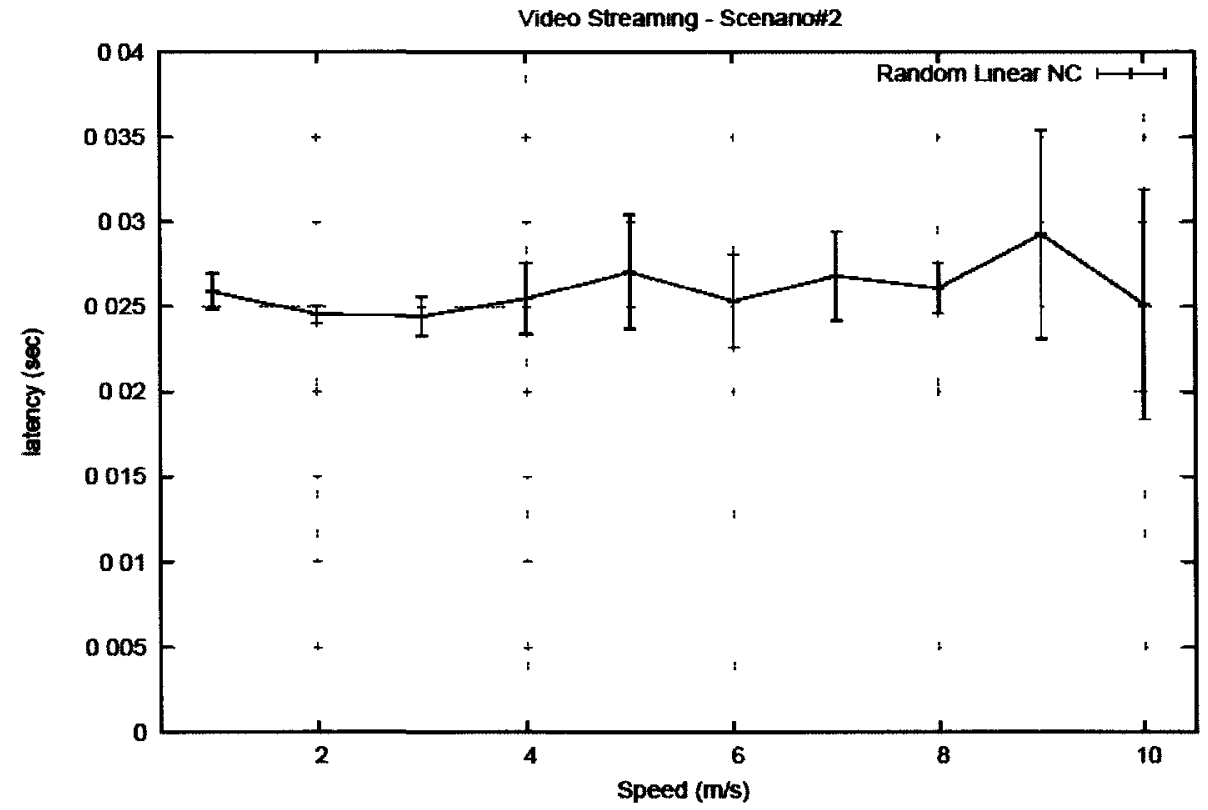

Figure 6.43: Latency for Video Streaming (Mobile Scenario - High Density) 


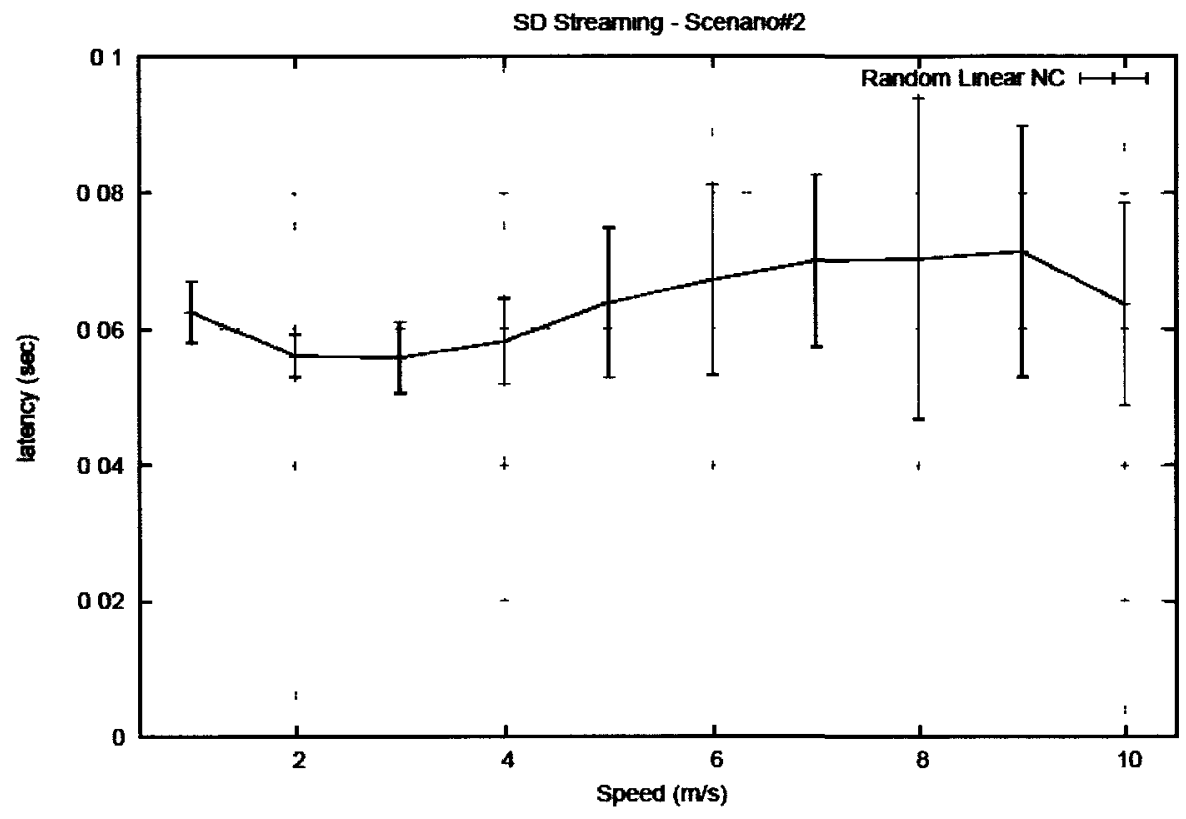

Figure 6.44: Latency for SDTV (Mobile Scenario - High Density)

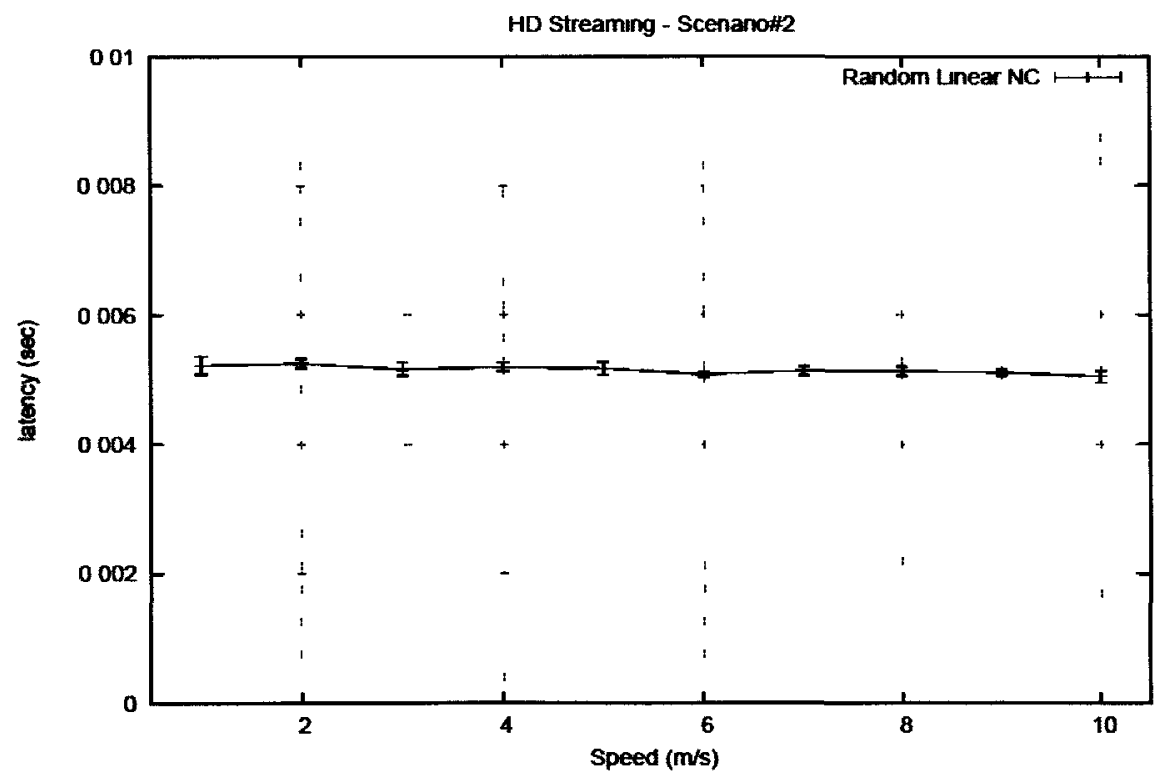

Figure 6.45: Latency for HDTV (Mobile Scenario - High Density) 
Figure 6.42, Figure 6.43, Figure 6.44 and Figure 6.45 show the latency results for Scenario\#2. Similar to scenario\#1 for high density networks, the latency values are higher compared to latency values in scenario\#2 for low density networks for all tested protocols. However, the latency values for RLNC remain the lowest and acceptable (below 200ms) for the multimedia broadcast applications that are used in the research.

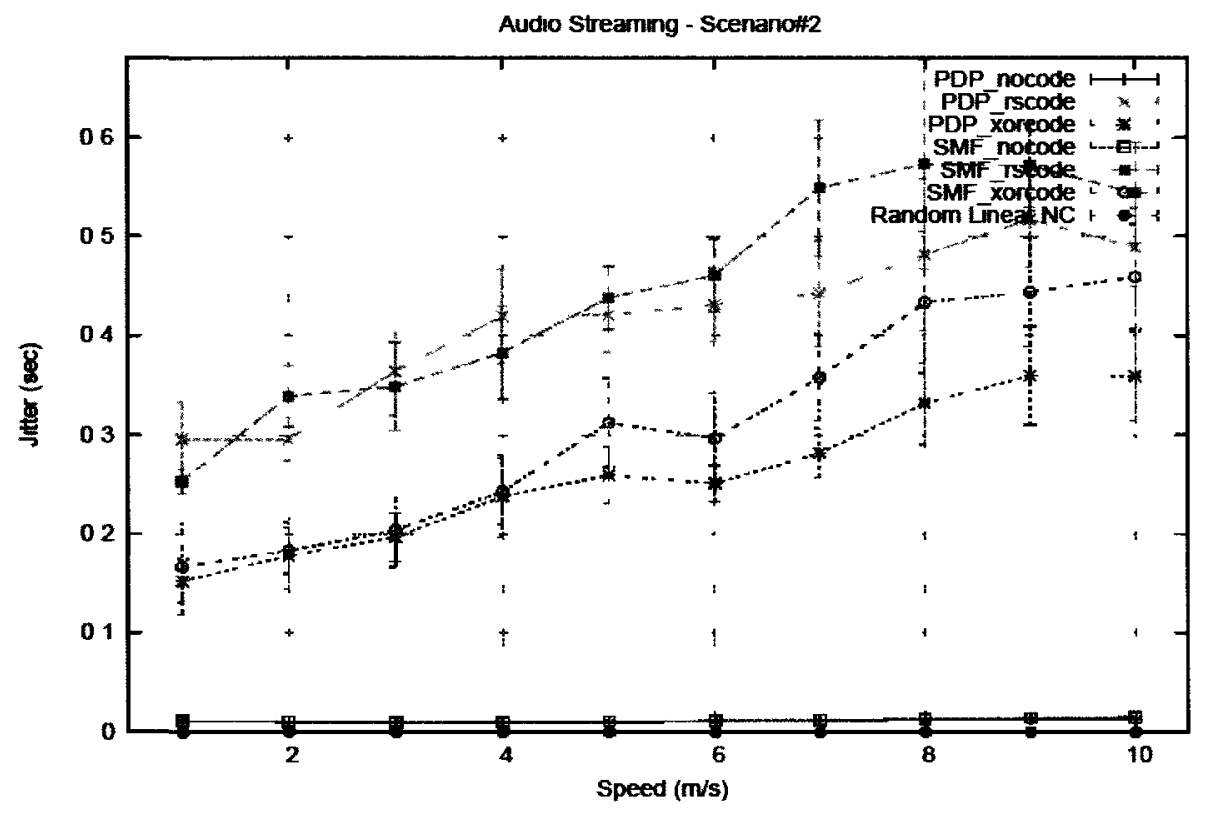

Figure 6.46: Jitter for Audio Streaming (Mobile Scenario - High Density) 


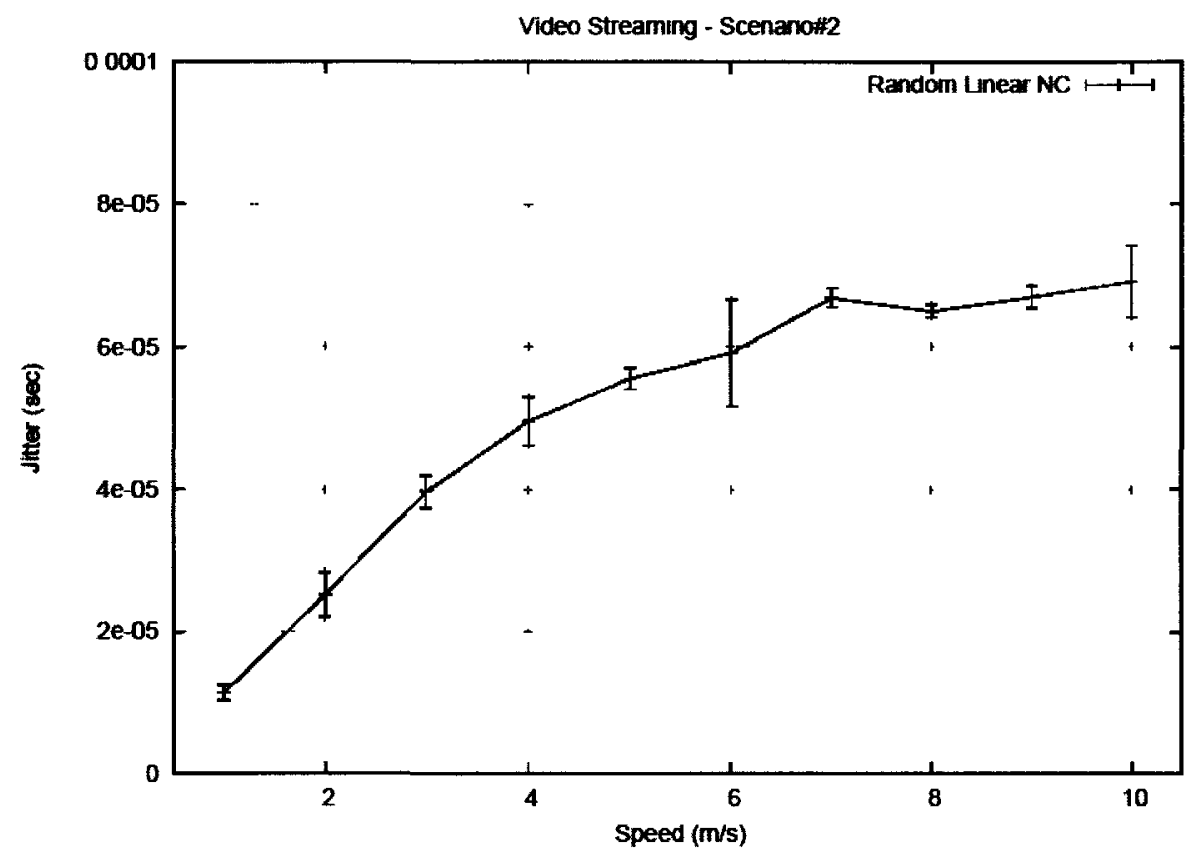

Figure 6.47: Jitter for Video Streaming (Mobile Scenario - High Density)

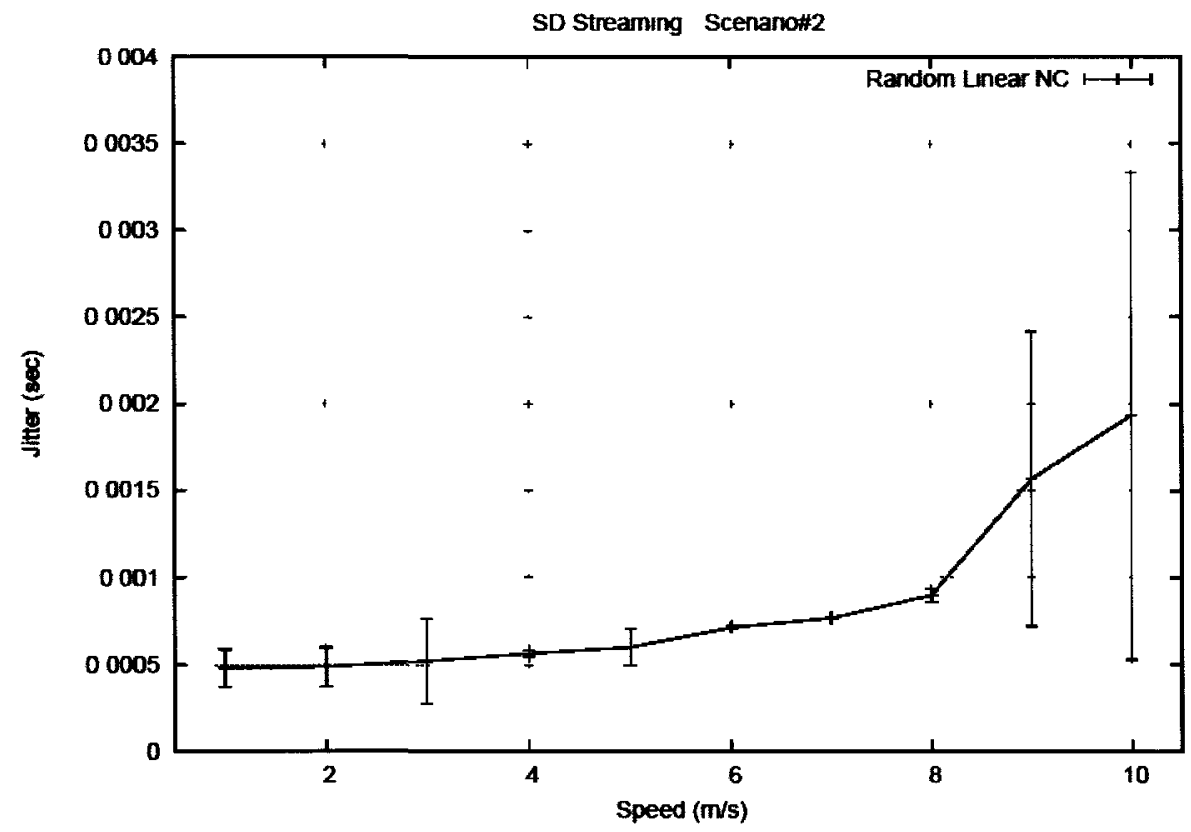

Figure 6.48: Jitter for SDTV (Mobile Scenario - High Density) 


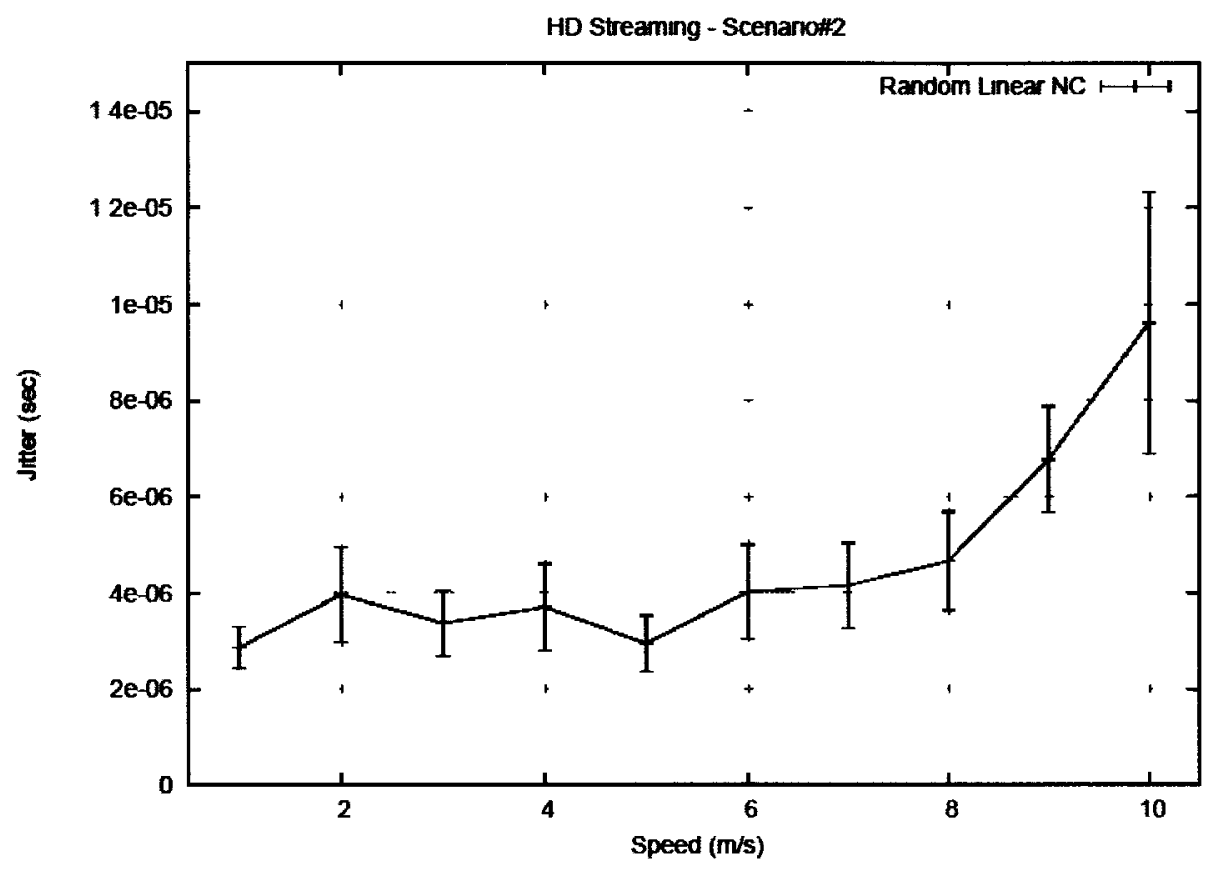

Figure 6.49: Jitter for HDTV (Mobile Scenario - High Density)

Figure 6.46, Figure 6.47, Figure 6.48 and Figure 6.49 show the jitter results for scenario $\$ 2$ for different multimedia broadcast applications. From Figure 6.46, it is clear that the jitter value of RLNC is very low over different speeds, because of the reason similar to the previous scenario. However, the jitter values of all tested protocols in this scenario increases by increasing the speed. When the speed increases, the wireless nodes in the network change their locations faster. This leads to increase the variance of the delivery time of the packets, as every time receivers received packets from different neighbor.

Based on the previous results, the best protocol to be used for multimedia broadcast applications is also the RLNC as it has the highest value of PDR and the lowest 
values of latency and jitter over different speeds. As a result, RLNC improves the overall performance of the multimedia broadcast applications in high density networks. 


\section{CHAPTER 7 - CONCLUSION AND FUTURE WORK}

This chapter concludes the thesis and provides some ideas for future work that could be useful to improve the performance of multimedia broadcast applications using $\mathrm{NC}$ techniques.

\subsection{Conclusion}

In this thesis, we explored ways to efficiently support multimedia broadcast applications. The thesis compared different optimized broadcast protocols and optionally with the use of NC with RLNC. The thesis also showed that the best protocol to be used for multimedia broadcast applications among all tested protocols is the RLNC protocol. The reason of this is that RLNC has the highest values of PDR, and the lowest values of latency and jitter. In summary, RLNC improves the overall performance of the multimedia broadcast applications, such as audio streaming, video streaming, SDTV and HDTV in low and high density as hoc wireless mesh networks, and in both static and mobile networks.

On the other hand, the use of XOR-NC and RS-NC techniques negatively affect the performance of the multimedia broadcast applications in ad hoc wireless mesh networks. In audio streaming, both XOR-NC and RS-NC negatively affect the over performance specifically in terms of latency and jitter; both latency and jitter are considered the two main performance criteria for the quality of multimedia broadcast applications. The latency and jitter results showed that both XOR-NC and RS-NC perform badly in high and low density ad hoc wireless mesh networks. In terms of mobility, increasing the speed increases jitter, but it has little effect on latency where the latency of 
both techniques are already high in audio streaming applications compared to RLNC and the optimized broadcast protocols. However, the results showed that XOR-NC and RS-NC techniques improve the PDR performance of audio streaming applications. On the other hand, XOR-NC and RS-NC perform badly in all other multimedia broadcast applications (video streaming, SDTV and HDTV) in terms of PDR, latency and jitter as the data rate and packet size increase in these applications.

The optimized broadcast protocols (SMF and PDP) without NC perform well for audio streaming applications with various node speeds in case of using up to two BSs in ad hoc wireless mesh networks. SMF and PDP protocols without NC also perform badly for multimedia broadcast applications with high data rates and large packet sizes, such as video streaming, SDTV and HDTV. The results showed that both SMF and PDP protocols have lower PDR, and much higher latency ( $>200 \mathrm{~ms}$ which is not acceptable for multimedia broadcast applications) and jitter than that of RLNC.

\subsection{Future Work}

During the work on this thesis, possibilities of future work have arisen. They are summarized as follows:

- Although the RLNC protocol is showing good performance in low and high density networks with multimedia applications, some of the factors are still static, such as forwarding factor and NC Timer (explained in 4.3). As a future work, some schemes can be developed to have dynamic factors that will depend upon the number of wireless nods and the data rate. The forwarding factor is the average number of encoded packets that a node can broadcast. The NC timer is 
the time needed to create and broadcast a newly encoded packet. Choosing a higher forwarding factor or a NC timer will increase the PDR results, but they may also increase the latency. Increasing forwarding factor or NC timer could be a practical solution for non-multimedia applications.

- In this thesis, those protocols were tested using two types of networks based on the number wireless nodes surrounding the BS(s); one network contains 1-4 BS(s) and ten wireless nodes and the other one contains 1-4 BS(s) and hundred wireless nodes. As a future work, a study can be performed using different types of scenarios by changing the number of wireless nodes, the mobility model, the number of BSs, and the locations of BSs and wireless nodes.

- In this thesis, those protocols were tested using IEEE 802.11. Those protocols can be tested and analyzed using Worldwide Interoperability for Microwave Access (WiMAX) technology or Long Time Evolution (LTE) technology, as both WiMAX and LTE are considered as upcoming technologies in the wireless communication field (especially LTE).

- In this thesis, the size of the queue for the decoded packets on the receivers is zero for all tested protocols. In other words, a decoded packet will be sent to the application layer immediately. As a future direction, some schemes could be implemented using a static or dynamic buffer size for receivers depending upon the data flow (e.g., data rates and packet sizes). The queue could improve the quality of multimedia applications by reducing the jitter. In other words, the performance results of RLNC protocols can be further improved for multimedia applications using such a dynamic scheme. 


\section{REFERENCES}

[1] R. Ahlswede, N. Cai, S.-Y. R. Li, R. W. Yeung, "Network Information Flow", Proc. of IEEE Transaction on Information Theory, vol. IT-46, pp. 1204-1216, 2000.

[2] R. Ducey, "Multimedia Broadcasting and the Internet", www.iif.hu/inet_96/b3/b3 2.htm, Accessed on March 4, 2011.

[3] S. R. Li, R. W. Yeung, N. Cai, "Linear Network Coding", Proc. of IEEE Transaction on Information Theory, vol. 49,no. 2, pp. 371-381, 2003.

[4] O. Oyman, "Enabling Mobile Video Services over WiMAX and LTE", a tutorial. Proc. of IEEE Vehicular Technology Society of the Institute of Electrical and Electronics Engineers, 2010.

[5] Arbitron/ Edison Media Research (2003), "Internet and Multimedia 11: New Media enters the mainstream", www.radiostreamingnews.com/2010/02/streaming-radiogrowth-charts.html, Accessed on March 8, 2011.

[6] J. Khan, R. Zaghal, "Jitter and Delay Reduction for Time Sensitive Elastic Traffic for TCP-interactive Based World Wide Video Streaming over ABone", Proc. of the 12th IEEE International Conf. on Computer Communications and Networks, pp. 311-318, 2003.

[7] T. Kunz, L. Li, "Broadcasting in Multihop Mobile Tactical Networks: To Network Code or Not", Proc. of the 6th International Wireless Communications and Mobile Computing Conference, pp. 676-680, 2010.

[8] E. Fasolo, M. Rossi, J. Widmer, M. Zorzi, "A Proactive Network Coding Strategy for Pervasive Wireless Networking", Proc. of IEEE GLOBECOM , pp. 5271-5276, 2007.

[9] E. Fasolo, M. Rossi, J. Widmer, M. Zorzi, "On MAC Scheduling and Packet Combination Strategies for Random Network Coding", Proc. of IEEE ICC , pp.3582-358, 2007.

[10] D. S. Lun, M. Médard, R. Koetter, "Efficient Operation of Wireless Packet Networks using Network Coding", Proc. of International Workshop on Convergent Technologies, pp. 1-5, 2005. 
[11] E. Ahmed, A. Eryilmaz, M. Médard, A. E. Ozdaglar, "On the Scaling Law of Network Coding Gains in Wireless Networks", Proc. of IEEE Military Communications Conference, 2007.

[12] R. Khalili, M. Ghaderi, J. Kurose and D. Towsley, "On the Performance of Random Linear Network Coding in Relay Networks", Proc. of IEEE Military Communication, pp. 1-7, 2008.

[13] S. Zhang, S.C. Liew, P. Lam, "Physical-Layer Network Coding", Proc. of the 12th in International Conference on Mobile Comp. \& Networking, pp. 358-365, 2006.

[14] M. Hay, B. Saeed, C.-H. Lung, and A. Srinivasan, "Co-Located Physical-Layer Network Coding to Mitigate Passive Eavesdropping", Proc. of the $8^{\text {th }}$ International Conference on Privacy, Security, and Trust , 2010.

[15] P. Popovski, H. Yomo, "Physical Network Coding in Two Way Wireless Relay Channels", Proc. of IEEE International Conference on Communications (ICC), 2007.

[16] P. Popovski and H. Yomo, "Wireless Network Coding by Amplify-and-Forward for Bi-directional Traffic Flows", IEEE Communication Letter,vol. 11, no. 1, pp. $1618,2007$.

[17] S. Katti, H. Rahul, W. Hu, D. Katabi, M. Médard and J. Crowcroft, "XORs in the Air: Practical Wireless Network Coding", Proc. of IEEE/ACM Transactions on Networking, pp. 497-510, 2008.

[18] J. Le, J. C. S. Lui, and D. M. Chiu, "DCAR: Distributed Coding-Aware Routing in Wireless Networks", Proc. of Distributed Computing Systems, 2008.

[19] K. Hamilton, (2000), "Polynomial Codes Over Certain Finite Fields", http://www.cs.cornell.edu/courses/cs722/2000sp/reedsolomon.pdf, Accessed on September 8, 2010.

[20] L. Li, R. Ramjee, M. Buddhikot, S. Miller, "Network Coding-Based Broadcast in Mobile Ad Hoc Networks", Proc. of IEEE International Conference on Computer Communications, 2007.

[21] T. Ho, M. Médard, R. Koetter, D. R. Karger, M. Effros, J. Shi, B. Leong, "A Random Linear Network Coding Approach to Multicast", Proc. of IEEE Transactions on Information Theory, vol. 52, p.p. 4413-4430, 2006. 
[22] P. Vingelmann ,P. Zanaty, FH. Fitzek, H. Charaf, "Implementation of random linear network coding on opengl-enabled graphics cards", Proc. of European Wireless, 2009.

[23] C. Fragouli and E. Soljanin, "Network coding: Fundamentals", Proc. of Foundations and Trends in Networking, vol. 2, pp. 1-133, 2007.

[24] P. A. Chou and Y. Wu, "Network coding for the Internet and wireless networks", Proc. of IEEE Signal Process. Mag., vol. 24, no. 5, pp. 77-85, 2007.

[25] K. Nguyen, T. Nguyen, and S. Cheung, "Peer-to-peer streaming with hierarchical network coding", Proc. of IEEE International Conference on Multimedia and Expo, pp. 396-399, 2007.

[26] C. Feng, B. Li, "Network Coding for Peer-Assisted Multimedia Streaming", Proc. of IEEE Communications Society, vol. 5, no. 2, pp. 9-12, 2010.

[27] T. Nguyen, "Network Coding for Wireless Video Communication", Proc. of IEEE Communications Society, vol. 5, no. 2, pp. 9-12, 2010.

[28] I. Lopetegui, R. Carrasco, S. Boussakta, "Experimental Measurements for VoIP with Network Coding in IEEE 802.11", Proc. of Wireless Communication Systems, pp. 795-799, 2010.

[29] J. Sang Park, M. Gerla, "Codecast: a Network-Coding-Based Ad hoc Multicast Protocol", Proc. of IEEE Wireless Communications, vol. 13, no.5, 2006.

[30] A. Argyriou, "Wireless Network Coding with Improved Opportunistic Listening", Proc. of IEEE Transactions on Wireless Communications, vol. 8, no.4, pp. 20142023, 2009.

[31] D. S. Lun, M. Medard, and R. Koetter, "Efficient Operation of Wireless Packet Networks Using Network Coding", Proc. of International Workshop on Convergent Technologies, 2005.

[32] Y. Kondo, H. Yomo, S. Yamaguchi, P. Davis, R. Miura, S. Obana, "Reliable Wireless Broadcast with Random Network Coding for Real-Time Applications", Proc. of IEEE Wireless Communications and Networking Conference, pp. 28402845, 2009. 
[33] M . Ghaderi, D. Towsley, J. Kurose, "Network Coding Performance for Reliable Multicast”, Proc. of IEEE Militray Communications Conference, 2007.

[34] H. Zhang, J. Zhou, Z. Chen and J. Li, "Minimizing Delay for Video Conference with Network Coding", Proc. of ACM Special Interest Group on Data Communication, 2009.

[35] L. Lima, S.Gheorghiu Gheorghiu, J. Barros, M. Médard, A.L. Toledo, "Secure Network Coding for Multi-Resolution Wireless Video Streaming", Proc. of IEEE Journal of Selected Areas in Comunications, vol. 28, no. 3, pp. 377-388, 2010.

[36] Q. Gu, J. Zhou, K. Ouyang, "An Approach of Scalable MPEG-4 Video Bitstreams with Network Coding for P2P Swarming System", nas, Proc. of IEEE International Conference on Networking, Architecture, and Storage, pp.239-242,2009.

[37] S. Xiao, H. Wang, C.-C. J. Kuo, "Priority Ordering and Packetization for Scalable Video Multicast with Network Coding", Proc. of the multimedia 8th Pacific Rim conference on Advances in multimedia information processing, 2007.

[38] E. Ahmed, A. Eryilmaz, A. Ozdaglar, and M. Medard, "On the Scaling Law of Network Coding Gains in Wireless Networks", Proc. of IEEE Military Communications, 2007.

[39] R. Khalili, M. Ghaderi, J. Kurose, D. Towsley, "On the Performance of Random Linear Network Coding in Relay Networks", Proc. of IEEE Military Communications, 2008.

[40] J. Macker, I. Downard, J. Dean, and B. Adamson, "Evaluation of Distributed Cover Set Algorithms in Mobile Ad hoc Network for Simplified Multicast Forwarding", Proc. of ACM Mobile Computing and Communications Review, vol. 11, no. 3, pp. 1-11, 2007.

[41] A. Rahman, M.E. Hoque, F. Rahman, S. K. Kundu, and P. Gburzynski, "Enhanced Partial Dominant Pruning (EPDP) Based Broadcasting in Ad hoc Wireless Networks", Proc. of Journal of Networks, vol. 4, no. 9, pp. 895-904, 2009.

[42] I. F. Akyildiz, X. Wang and W. Wang, "Wireless Mesh Networks: a Survey", Proc. of Computer Networks and ISDN Systems, vol.47 no. 4, pp.445-487, 2005

[43] A. Zimmermann, M. Güne, M. Wenig, U. Meis and J. Ritzerfeld, "How to Study Wireless Mesh Networks: A hybrid Testbed Approach", Proc. of the 21st IEEE 
International Conference on Advanced Information Networking and Applications. pp. 853-860, 2007.

[44] R. Manoharan and E. Ilavarasan, "Impact of the Mobility on the Performance of Multicast Routing Protocols in MANET", Proc. of International Journal of Wireless and Mobile Networks, vol. 2 no. 2, 2010.

[45] “MPEG-2 Systems", Available, http://www.chiariglione.org/faq/mp2sys/mp2sys .htm\#mp2-12, Accessed on December 8, 2010.

[46] "IEEE 802 Tutorial: Video over 802.11", http://www.ieee802.org/802tutorials/ index.htm, Viewed 2010 December 10.

[47] "BonnMotion Mobility Generator", www.informatik.unibonn.de/IV/BonnMotion, Accessed on September 4, 2010.

[48] "Routing Protocols in MANEET", International Journal of wireless \& mobile networks (IJWMN), vol.2 no.2, 2010, pp 110-119. 


\section{APPENDIX A}

Network Topology Codes:

set ns__ [new Simulator]

Figure A.1: NS Object Creation

set tracefd [open out.tr $w$ ]

sns_trace-all stracefd

Figure A.2: Trace File Creation

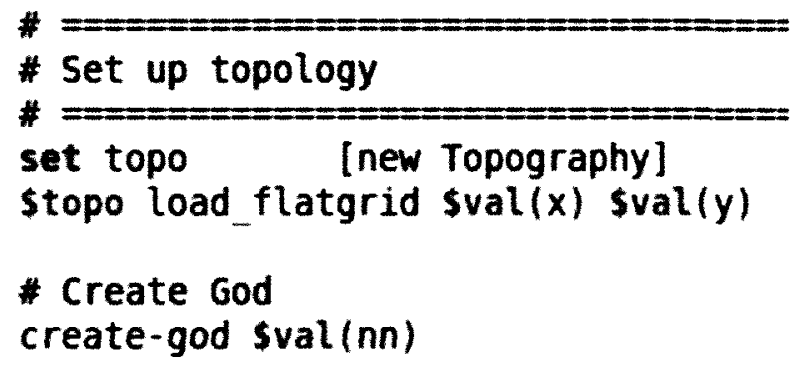

Figure A.3: God and Topology Creation

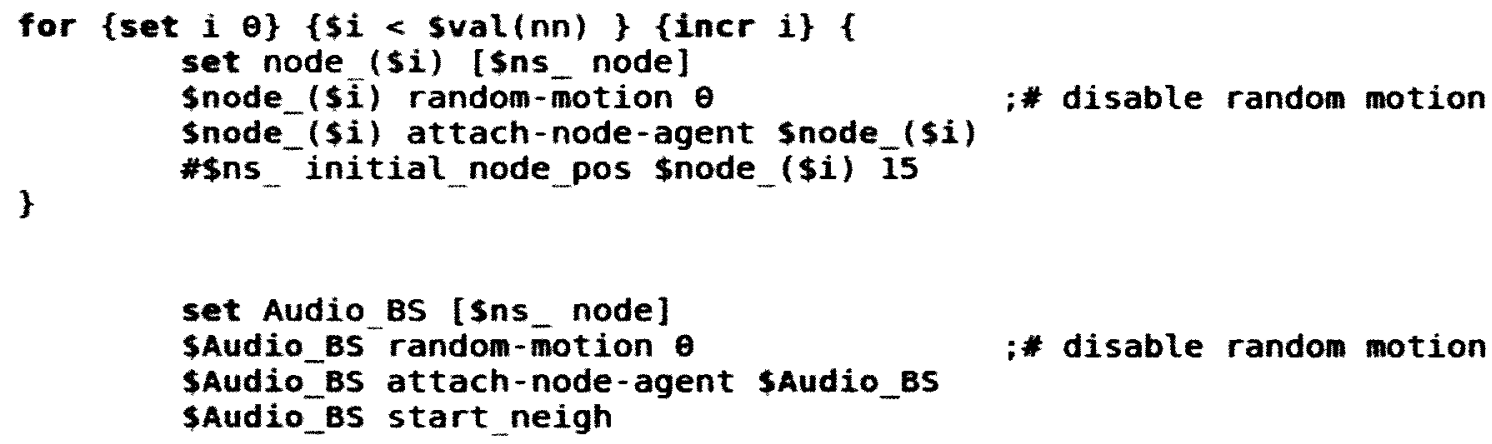

Figure A.4: Base Station and Nodes Creation 
set chan_1_ [new sval(chan)]

$$
\begin{aligned}
& \text { sns node-config -adhocRouting \$val(rp) I } \\
& \text { - llType \$val(Ll) । } \\
& \text { - macType \$val (mac) । } \\
& \text {-ifqType \$val (ifq) । } \\
& \text { - ifqLen sval(ifqlen) । } \\
& \text {-antType sval(ant) I } \\
& \text {-proptype \$val(prop) I } \\
& \text {-phyType sval(netif) । } \\
& \text { - channel \$chan_1 I } \\
& \text { - topoInstance \$topo । } \\
& \text { - agentTrace OFF I } \\
& \text { - routerTrace OFF । } \\
& \text {-macTrace OFF । } \\
& \text { A } \\
& \text {-movementTrace OFF }
\end{aligned}
$$

Figure A.5: PDP Base Station and Nodes Creation

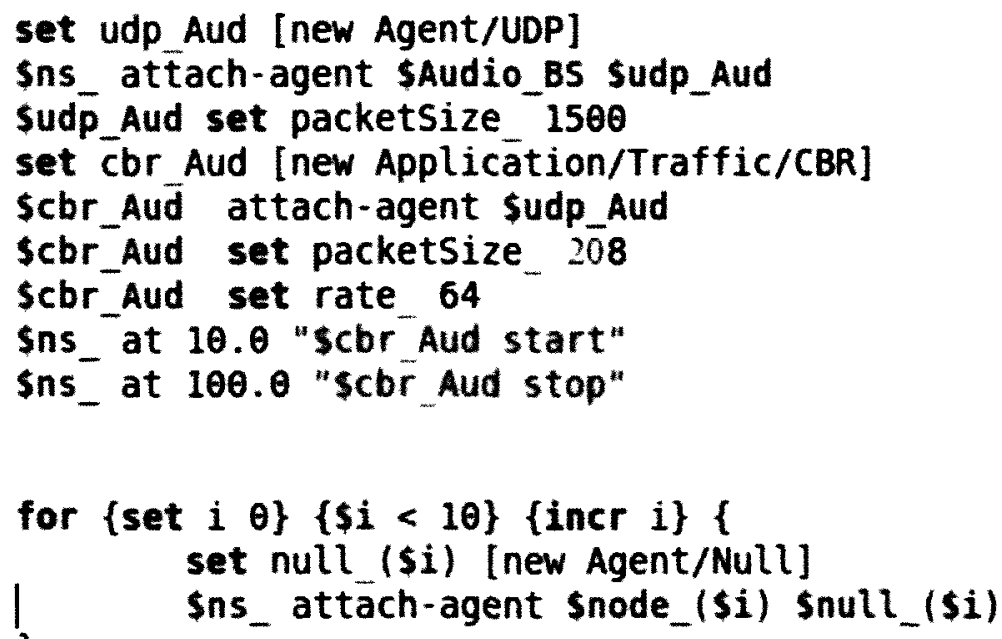

Figure A.6: Audio Streaming Traffic

\$udp_Aud set dst_addr_oxE100000

sudp_Aud set dst_port_ 699

Figure A.7: PDP and SMF Broadcast Address and Port

$$
\begin{aligned}
& \text { \$ns_at } 10 . \theta^{\prime} \text { "\$cbr_Aud start" } \\
& \text { \$ns_at } 10 \theta . \text { " }^{\text {\$cbr_Aud stop" }}
\end{aligned}
$$

Figure A.8: Start and Stop Traffic Broadcasting 
Protocols' Codes:

$$
\begin{aligned}
& \text { Agent/CODEBCAST set hello_int_ } 1 \\
& \text { Agent/CODEBCAST set PDP true } \\
& \text { Agent/CODEBCAST set coding fales } \\
& \text { Agent/CODEBCAST set RS_coding_fales }
\end{aligned}
$$

Figure A.9: PDP Specifications

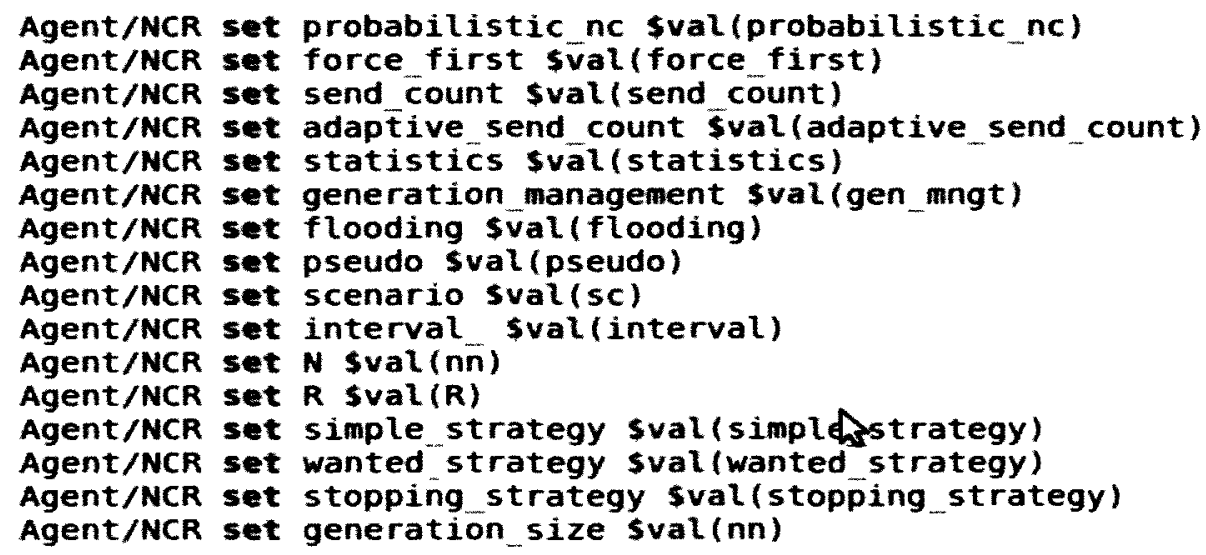

Figure A.10: RLNC Specifications

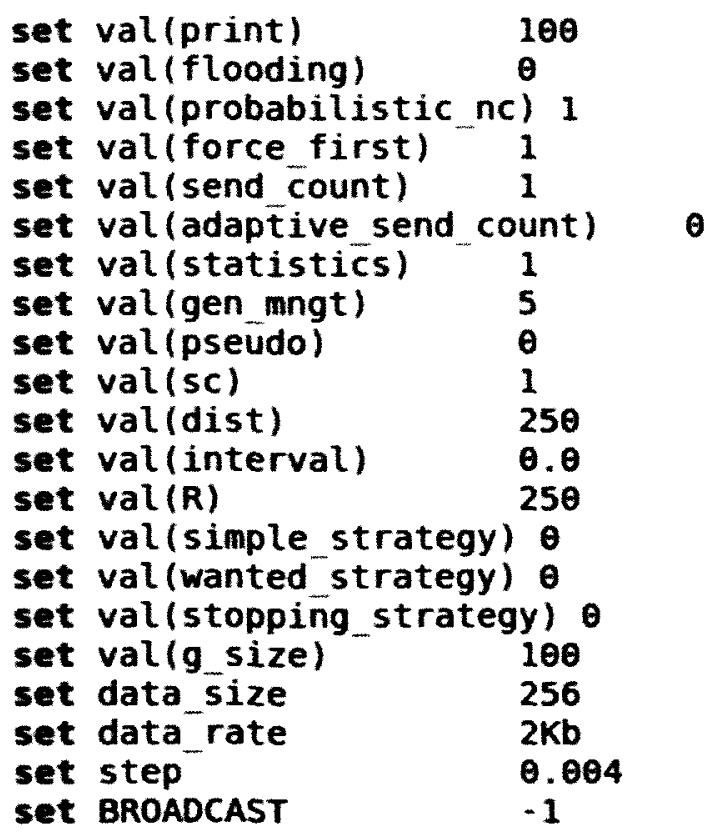

Figure A.11: RLNC Specifications Values 